Prepared for the U.S. Department of Energy

under Contract DE-AC05-76RL01830

\title{
Online Analysis of Wind and Solar Part I: Ramping Tool
}
PV Etingov
YV Makarov
$\mathrm{J} \mathrm{Ma}$
K Subbarao

January 2012

Pacific Northwest

NATIONAL LABORATORY

Proudly Operated by Battelle Since 1965 



\title{
DISCLAIMER
}

United States Government. Neither the United States Government nor any agency thereof, nor Battelle Memorial Institute, nor any of their employees, makes any warranty, express or implied, or assumes any legal liability or responsibility for the accuracy, completeness, or usefulness of any information, apparatus, product, or process disclosed, or represents that its use would not infringe privately owned rights. Reference herein to any specific commercial product, process, or service by trade name, trademark, manufacturer, or otherwise does not necessarily constitute or imply its endorsement, recommendation, or favoring by the United States Government or any agency thereof, or Battelle Memorial Institute. The views and opinions of authors expressed herein do not necessarily state or reflect those of the United States Government or any agency thereof.

\author{
PACIFIC NORTHWEST NATIONAL LABORATORY \\ operated by \\ BATTELLE \\ for the \\ UNITED STATES DEPARTMENT OF ENERGY \\ under Contract DE-AC05-76RL01830 \\ Printed in the United States of America \\ Available to DOE and DOE contractors from the \\ Office of Scientific and Technical Information, \\ P.O. Box 62, Oak Ridge, TN 37831-0062; \\ ph: (865) 576-8401, fax: (865) 576-5728 \\ email: reports@adonis.osti.gov \\ Available to the public from the National Technical Information Service, \\ U.S. Department of Commerce, 5285 Port Royal Rd., Springfield, VA 22161 \\ ph: (800) 553-6847, fax: (703) 605-6900 \\ email: orders@ntis.fedworld.gov \\ online ordering: http://www.ntis.gov/ordering.htm
}

This document was printed on recycled paper.

$(8 / 00)$ 


\section{ACKNOWLEDGEMENTS}

This work was funded by the Public Interest Energy Research (PIER) program in renewable energy at the California Energy Commission and managed by the California Institute for Energy and Environment. This report was prepared with substantial support from California Independent System Operator personnel.

The authors would like to thank Mike Gravely, Pedro Gomez, and Jamie Patterson, California Energy Commission; Merwin Brown, Larry Miller, and Jim Cole, California Institute for Energy and Environment; and Sirajul Chowdhury, Zhijun (June) Xie, and Ajay Mannepalli, California Independent System Operator, for providing the project team with consultations, day-by-day support, and discussions essential to this work.

The authors would also like to thank Managers Carl H. Imhoff, Dale King, Evan O. Jones, Mark P. Morgan, Ron Melton, and Landis Kannberg, Staff Scientists Dr. Ryan Hafen, Michael T. Batdorf, John C. Bower (retired), Contracting Officer Sheena Kanyid, Project Specialist Susan Arey, and Administrative Assistant Nikki Stringer, Pacific Northwest National Laboratory, for supporting this work.

Many thanks go to Technical Writers Maura K. Zimmerschied and Kathy Neiderhiser, Pacific Northwest National Laboratory, for editing the final manuscript.

\section{DISCLAIMER}

This report was prepared as the result of work sponsored by the California Energy Commission. It does not necessarily represent the views of the Energy Commission, its employees or the State of California. The Energy Commission, the State of California, its employees, contractors and subcontractors make no warrant, express or implied, and assume no legal liability for the information in this report; nor does any party represent that the uses of this information will not infringe upon privately owned rights. This report has not been approved or disapproved by the California Energy Commission nor has the California Energy Commission passed upon the accuracy or adequacy of the information in this report. 


\title{
Online Analysis of Wind and Solar Part I: Ramping Tool
}

\author{
PV Etingov \\ $\mathrm{J}$ Ma \\ YV Makarov \\ K Subbarao
}

January 2012

Prepared for

California Institute for Energy and Environment and the California Energy Commission

via U.S. Department of Energy

Contract DE-AC05-76RL01830

Related Services

Pacific Northwest National Laboratory

Richland, Washington 99352 



\section{PREFACE}

The California Energy Commission Public Interest Energy Research (PIER) Program supports public interest energy research and development that will help improve the quality of life in California by bringing environmentally safe, affordable, and reliable energy services and products to the marketplace.

The PIER Program conducts public interest research, development, and demonstration (RD\&D) projects to benefit California. The PIER Program strives to conduct the most promising public interest energy research by partnering with RD\&D entities, including individuals, businesses, utilities, and public or private research institutions.

PIER funding efforts are focused on the following RD\&D program areas:

- Buildings End-Use Energy Efficiency

- Energy Innovations Small Grants

- Energy-Related Environmental Research

- Energy Systems Integration

- Environmentally Preferred Advanced Generation

- Industrial/Agricultural/Water End-Use Energy Efficiency

- Renewable Energy Technologies

- Transportation

This report is the final report for the "Online Analysis of Wind and Solar" project (contract number: 500-07-537, work authorization number: POTPO1-X11) conducted by the Pacific Northwest National Laboratory. The information from this project contributes to PIER's Energy Systems Integration program area.

For more information about the PIER Program, please visit the Energy Commission's website at http://www.energy.ca.gov/research/, or contact the Energy Commission at 916-654-4878. 



\section{ABSTRACT}

The power system balancing process, which includes the scheduling, real-time dispatch (load following) and regulation processes, is traditionally based on deterministic models. Since the conventional generation needs time to be committed and dispatched to a desired megawatt level, the scheduling and load following processes use forecasts of load and wind and solar power production to achieve future balance between the conventional generation and energy storage on the one side, and system load, intermittent resources (such as wind and solar generation), and scheduled interchange on the other side. Although in real life the forecasting procedures imply some uncertainty around the load and wind/solar forecasts (caused by forecast errors), only their mean values are actually used in the generation dispatch and commitment procedures. Since the actual load and intermittent generation can deviate from their forecasts, it becomes increasingly unclear (especially, with the increasing penetration of renewable resources) whether the system would actually be able to meet the conventional generation requirements within the look-ahead horizon, what additional balancing efforts would be needed as we approach real time, and what additional costs would be incurred by those needs.

To improve the system control performance characteristics, maintain system reliability, and minimize expenses related to the system balancing functions, it becomes necessary to incorporate the predicted uncertainty ranges into the scheduling, load following, and, to some extent, into the regulation processes. It is also important to address the uncertainty problem comprehensively by considering all sources of uncertainty (load, intermittent generation, generators' forced outages, etc.) in consideration. All aspects of uncertainty, such as the imbalance size (which is the same as the capacity needed to mitigate the imbalance) and generation ramping requirement, must be taken into account. The latter unique features make this work a significant step forward toward the objective of incorporating wind, solar, load, and other uncertainties into power system operations.

Currently, uncertainties associated with wind and load forecasts, as well as uncertainties associated with random generator outages and unexpected disconnection of supply lines, are not taken into account in power grid operation. Thus, operators have few resources to weigh the likelihood and magnitude of upcoming events of power imbalance. In this project, funded by the California Energy Commission, a framework has been developed for incorporating uncertainties associated with wind, solar and load forecast errors, unpredicted ramps, and forced generation disconnections into the energy management system as well as generation dispatch and commitment applications.

A new approach to evaluating the uncertainty ranges for the required generation performance envelope, including balancing capacity, ramping capability, and ramp duration, is proposed and implemented. The approach includes three stages: forecast and actual data acquisition, statistical analysis of retrospective information, and prediction of future grid balancing requirements for specified time horizons and confidence levels. Assessment of the capacity and ramping requirements is performed using a specially developed probabilistic algorithm based on a histogram analysis, incorporating all sources of uncertainties, both continuous (wind and 
load forecast errors) and discrete (forced generator outages and start-up failures). A new method called the "flying brick" technique has been developed to evaluate the look-ahead required generation performance envelope for the worst-case scenario within a user-specified confidence level. A self-validation algorithm has been developed to validate the accuracy of the confidence intervals.

To demonstrate the validity of the developed uncertainty assessment methods and its impact on grid operation, a framework for integrating the proposed methods with California Independent System Operator (CAISO) market design has been developed. The software production tool has been developed and was installed at the CAISO control room in May 2011 for testing and benchmarking. The tool received very positive feedback from the CAISO specialists.

Keywords: Regulation reserve requirement, area control error, ancillary services, Control Performance Standards, Balancing Authority ACE (Area Control Error) Limit: BAAL, solar generation, load forecast, wind generation forecast, probability density function, swinging door algorithm.

Please use the following citation for this report:

Makarov, Yuri V., Pavel V. Etingov, Krishnappa Subbarao, Jian Ma (Pacific Northwest National Laboratory), and Clyde Loutan (CAISO). 2011. Online Analysis of Wind and Solar, Final Project Report. California Energy Commission. CEC-500-2011-XXX. 


\section{TABLE OF CONTENTS}

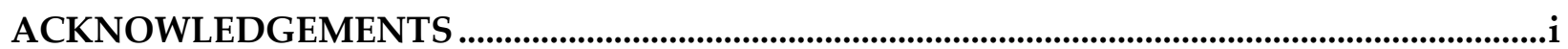

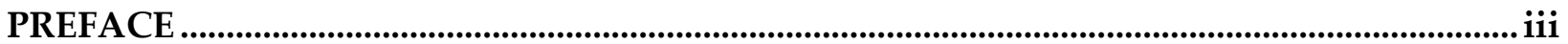

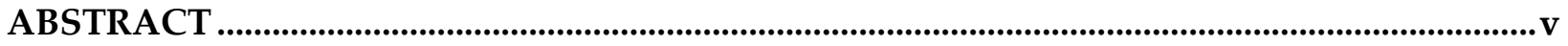

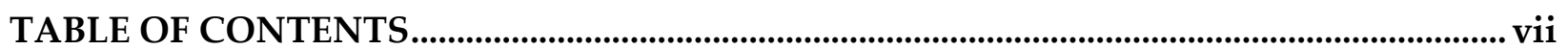

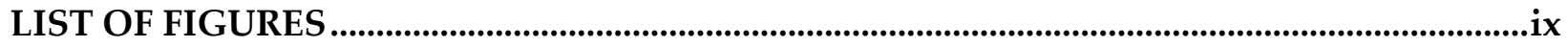

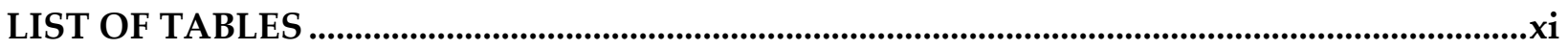

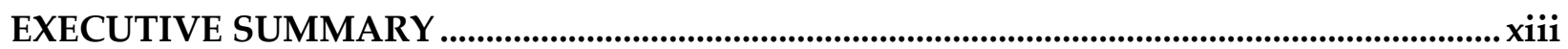

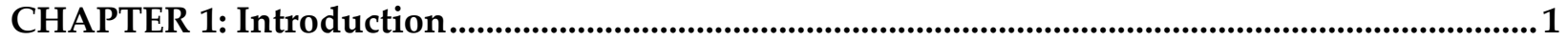

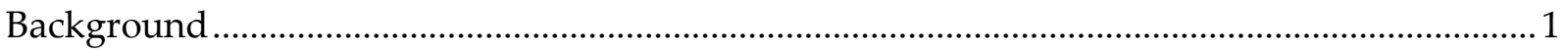

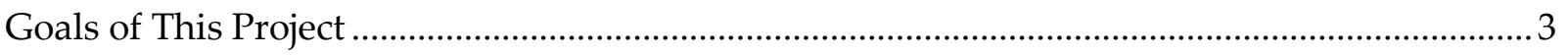

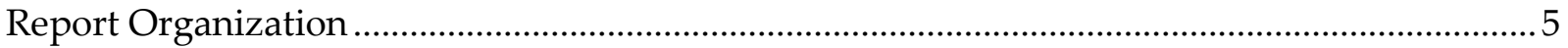

CHAPTER 2: Load and Wind/Solar Generation Uncertainty Evaluation ....................................... 6

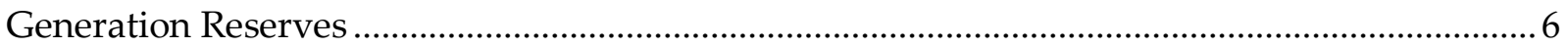

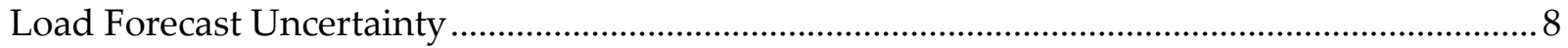

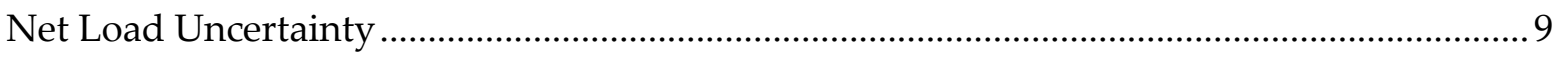

Statistical Methods to Evaluate the Forecast Uncertainty …..................................................... 9

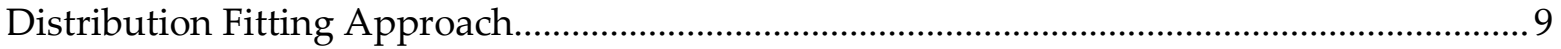

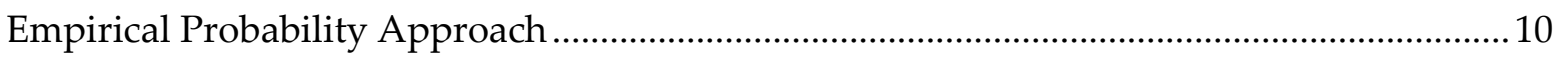

Assessment of the Generation Capacity Uncertainty …......................................................... 11

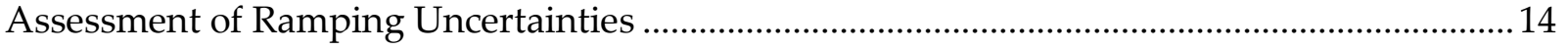

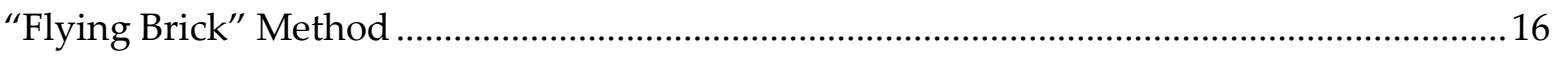

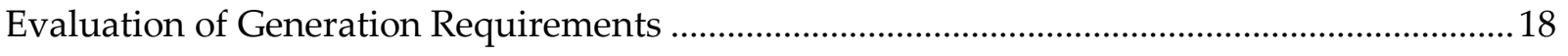

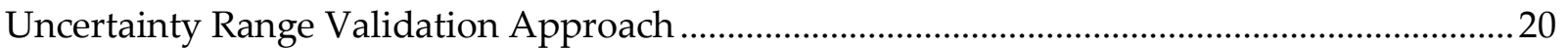

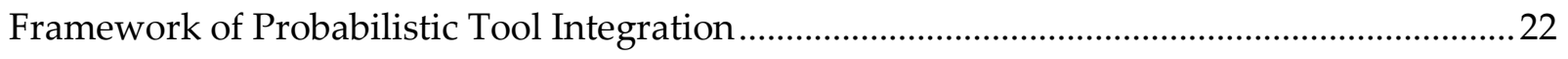

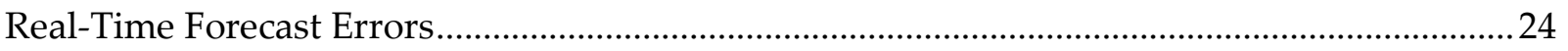

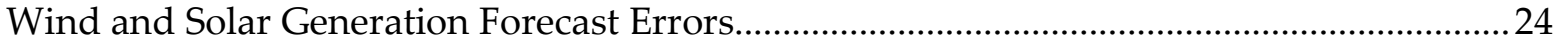

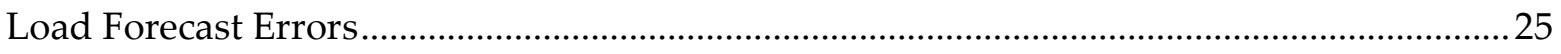


CHAPTER 3: Review of Current Operating Practices at CAISO .....................................................26

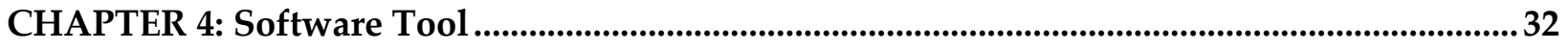

User Interface Conceptual Design .......................................................................................... 32

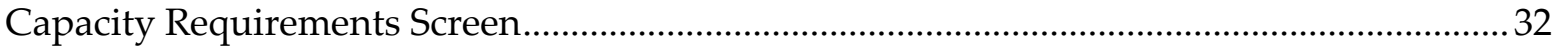

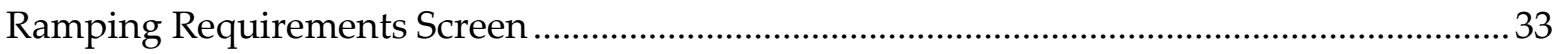

Conceptual Design of Probabilistic Tool Integration................................................................. 34

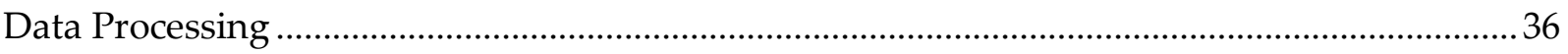

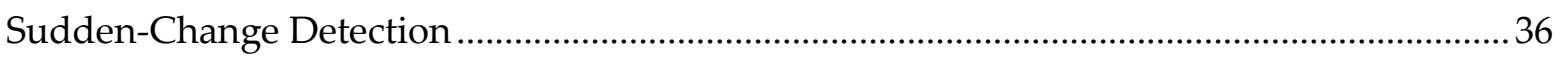

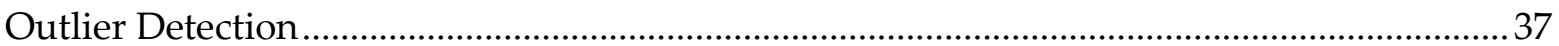

Straight-Line Detection Algorithm .................................................................................... 37

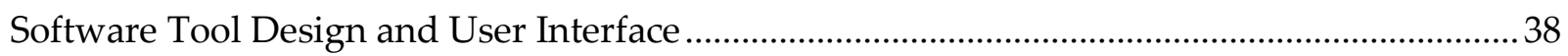

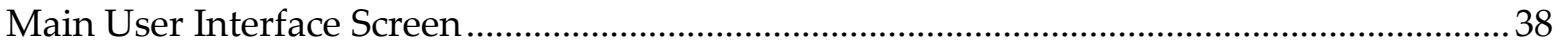

Test Plan for the Ramp Tool Prototype ...................................................................................... 41

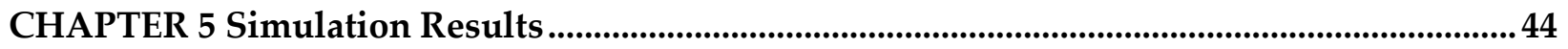

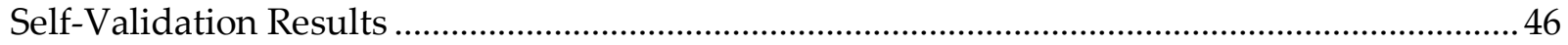

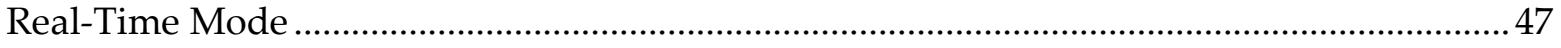

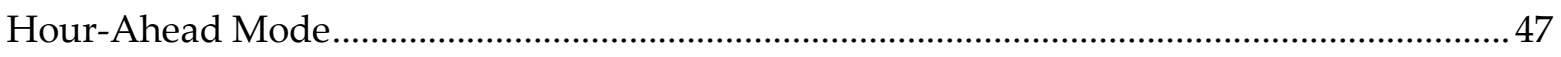

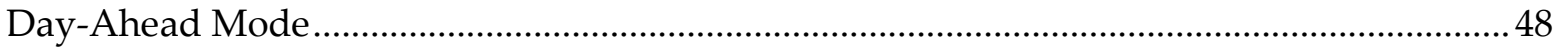

Prediction of Real-Time Market Price Spikes............................................................................ 48

Real-Time Ramping Requirements..................................................................................... 49

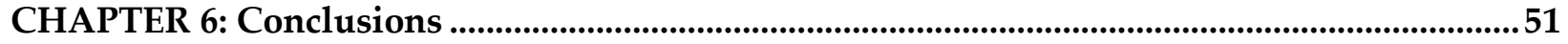

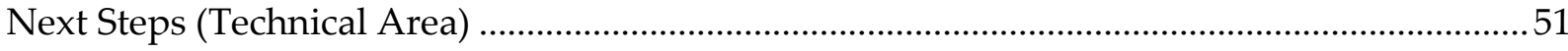

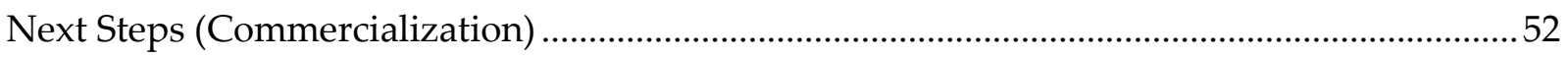

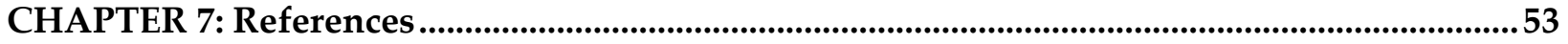

CHAPTER 8: Glossary....................................................................................................................................55

APPENDIX A : Generation Fleet Ramping Capability ….............................................................5 57

APPENDIX B : Generator Forced Outage Model ..............................................................................61 


\section{LIST OF FIGURES}

Figure 1: Probabilistic Display Concept.........................................................................................

Figure 2: Probabilistic Assessment Methodology Concept..................................................................

Figure 3: "Flying Brick" Method............................................................................................................

Figure 4: Software Tool GUI......................................................................................................

Figure 5: Real-Time Capacity Requirements Prediction (6/24/11 16:15) ....................................xviii

Figure 6: CAISO Real-Time Price $(6 / 24 / 11)$.................................................................................. xviii

Figure 7: "Active" and "Proactive" Integration Approaches ............................................................xix

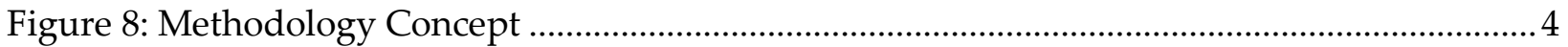

Figure 9: Allocation of Generation Unit Capacity .....................................................................

Figure 10: Load Fluctuation and Uncertainty: a) Day-Ahead Load Forecast vs. Actual Load;

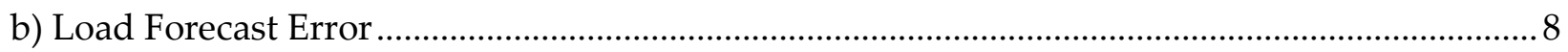

Figure 11: Load Forecast Error Histogram .................................................................................. 10

Figure 12: Net Load Forecast Error Distribution: a) Histogram; b) Empirical CDF ......................11

Figure 13: Statistical Characteristics of Wind Generation Forecast for Different Look-Ahead

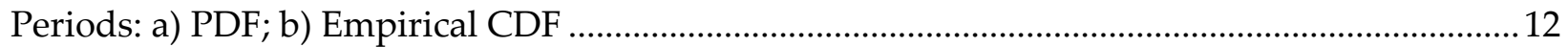

Figure 14: Wind Generation Forecast Inverse CDFs for Different Look-Ahead Intervals and

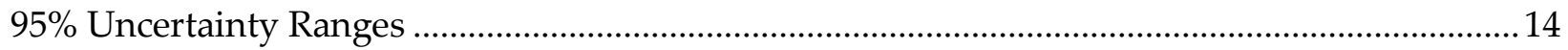

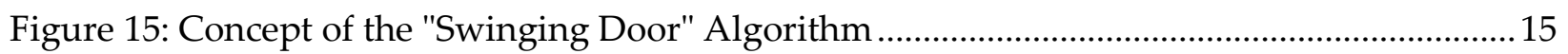

Figure 16: "Swinging Door" Algorithm - Obtaining Capacity, Ramp, and Ramp Duration ........ 15

Figure 17: Concurrent Consideration of the Capacity, Ramping and Duration Requirements.... 16

Figure 18. Concept of the "Flying Brick" Method ........................................................................... 17

Figure 19: Ramping Requirement PDFs for Different Ramp Durations ....................................... 18

Figure 20: Ramping Requirement Inverse CDFs for Different Ramp Durations and

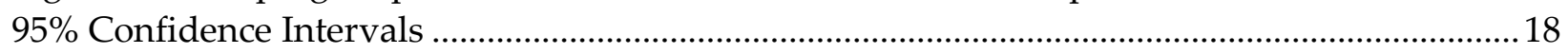

Figure 21: Evaluation of Capacity Requirements ..........................................................................20

Figure 22: Validation Procedure .............................................................................................. 21

Figure 23: Flowchart of EMS Integration for the Uncertainty Model...........................................23

Figure 24: Flowchart of Proactive Integration.............................................................................24 


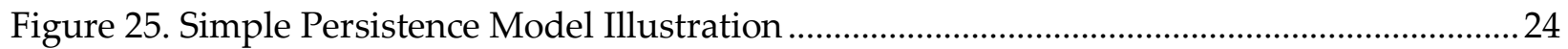

Figure 26: Real-Time Wind Generation Forecast........................................................................... 25

Figure 27: Real-Time Solar Generation Forecast.......................................................................... 25

Figure 28. Real-Time Load Forecast Error ........................................................................................ 25

Figure 29: An illustration of CAISO Operating Timelines (not to scale) ......................................26

Figure 30: A detailed illustration of CAISO Operating Timelines .................................................2 28

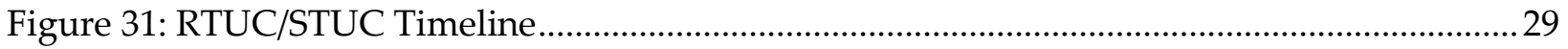

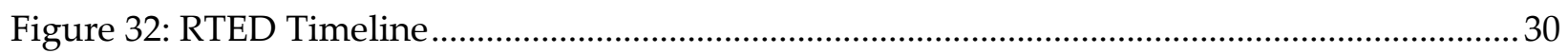

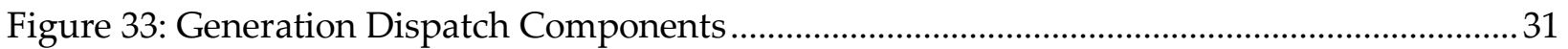

Figure 34: Capacity Requirements Display Concept ..................................................................... 33

Figure 35: Ramping Requirements Display Concept.................................................................... 34

Figure 36: CAISO Capacity Requirements Uncertainty Evaluation Concept ............................... 35

Figure 37: Example of Wind/Load/Solar Forecast Structure ............................................................36

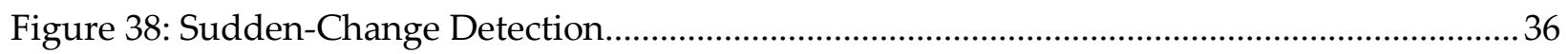

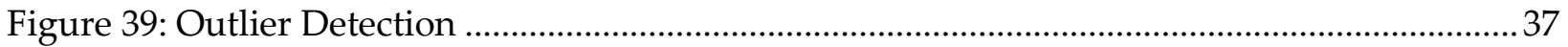

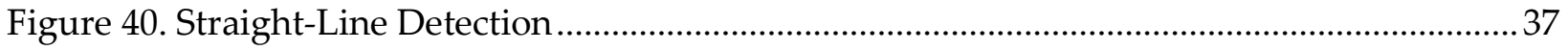

Figure 41: Screenshot of Day-Ahead Capacity Requirements Display .........................................39

Figure 42: Screenshot of Real-Time Requirements Display …....................................................... 39

Figure 43: Screenshot of Day-Ahead Ramping Requirements Display........................................ 40

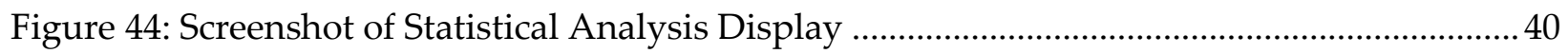

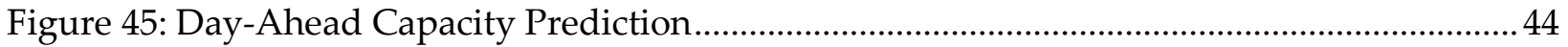

Figure 46: Day-Ahead Ramping Requirements Prediction..........................................................45

Figure 47: Hour-Ahead Capacity Requirements Prediction .......................................................... 46

Figure 48: Hour-Ahead Ramping Requirements Prediction ......................................................... 46

Figure 49: Real-Time Mode Self-Validation Results ....................................................................... 47

Figure 50: Hour-Ahead Mode Self-Validation Results ................................................................. 47

Figure 51: Day-Ahead Mode Self-Validation Results ................................................................... 48

Figure 52: Real-Time Capacity Requirements Prediction ......................................................... 49 
Figure 53: CAISO Real-Time Price*

Figure 54: Real-Time Ramping Requirements .......................................................................... 50

\section{LIST OF TABLES}

Table 1: Example of Validation Table (1-Hour Ahead Forecast)...................................................22

Table 2: Time Characteristics of the Scheduling Process at CAISO ...............................................27 



\section{EXECUTIVE SUMMARY}

This research was conducted by Pacific Northwest National Laboratory (PNNL) for the California Energy Commission and the California Institute for Energy and Environment.

Because conventional generators need time to be committed and dispatched to a desired megawatt level, scheduling and load following processes use load and wind power production forecasts to achieve future balance between conventional generation and energy storage on the one side and system load, intermittent resources (such as wind and solar generation), and scheduled interchange on the other side. The power system balancing process, which includes scheduling, real-time dispatch (load following), and regulation processes, is traditionally based on deterministic models.

Uncertainties in forecasting the output of intermittent resources such as wind and solar generation, as well as system loads, are not reflected in an existing energy management system (EMS) or tools for generation commitment, dispatch, and market operation. With the growing penetration of intermittent resources, these uncertainties could result in significant unexpected load-following and dispatch problems, and pose serious risks to control and operation performance characteristics as well as the reliability of a power grid. Without knowing the risks posed by the uncertainties, system operators have limited means to weigh the likelihood of occurrence and the magnitude of problems to mitigate adverse impacts caused by them. Some important questions need to be addressed in counteracting the impact of uncertainties; for instance, whether and when one should start more units to balance against possible fast ramps in the future over a given time horizon.

Furthermore, these uncertainties could require procuring additional costly balancing services. Major unexpected variations in wind power, unfavorably combined with load forecast errors and forced generator outages could cause significant power mismatches, which could be essentially unmanageable without knowing these variations in advance.

Because the actual load and intermittent generation can deviate from forecasts, it becomes increasingly unclear (especially with the increasing penetration of renewable resources) whether the system would be actually able to meet the conventional generation requirements within the look-ahead horizon, what additional balancing efforts would be needed as we approach real time, and what additional costs would be incurred by those needs.

In order to improve the system control performance characteristics, maintain system reliability, and minimize expenses related to the system balancing functions, it becomes necessary to incorporate the projected uncertainty ranges into the scheduling, load following, and, to some extent, into the regulation processes. This need has been realized already, and some wind forecast service providers offer uncertainty information for their forecasts. Various researchers are developing methodologies and tools to incorporate these uncertainties into power system operations. Unfortunately, in many cases, these efforts are limited to wind generation uncertainties only and ignore the fact that there are additional sources of uncertainty such as system loads and forced generation outages. Most of these efforts consider only the uncertainty ranges for the megawatt imbalances and do not address additional essential characteristics such 
as ramps and ramp duration uncertainties. This limited consideration could be misleading to power system operators responsible for system reliability and control performance characteristics.

It is very important to address the uncertainty problem comprehensively by including all sources of uncertainty (load, intermittent generation, generators' forced outages, etc.) into consideration. All aspects of uncertainty, such as the imbalance size (which is the same as the capacity needed to mitigate the imbalance) and generation ramping requirement, must be taken into account. The latter unique features make this work a significant step forward toward the objective of incorporating wind, solar, load, and other uncertainties into power system operations.

In this project, all uncertainties associated with wind power generation forecasting, load demand forecasting, and generation supply interruptions caused by forced outages are taken into account in the evaluation of uncertainty ranges for the required generation performance envelope including balancing capacity, ramping capability, and ramp duration. A probabilistic algorithm, based on a histogram analysis to assess the capacity and ramping requirements, is presented. Simulation was performed using California Independent System Operator (ISO)'s system model and data. This report also presents these simulation results confirming the validity and efficiency of the proposed solutions.

The work pursues the following objectives:

- Develop a probabilistic model to evaluate uncertainties of wind and load forecast errors and to provide rapid (every 5 minutes) look-ahead (up to 5-8 hours ahead) assessments of their uncertainty ranges.

Figure 1 shows the conceptual picture of the probabilistic capacity requirements display. The light and dark orange areas represent the evaluated capacity ranges for different confidence levels. The blue curve is the generation schedule. The gray area is the available balancing reserve, which is calculated from the margin of online generators. The system would have adequate balancing reserve with a specified confidence level if the available balancing reserve (the gray area) covers the entire net load uncertainty range (the dark or light orange area). Otherwise, deficiency of balancing reserve occurs. This means that there is a certain probability that the online generation will not be able to follow the net load requirement. In this case it is necessary to commit or de-commit additional generators to achieve the desired confidence level for the balancing reserve.

- Elaborate similar models to evaluate uncertainties caused by generator random forced outages, failures to start up, and contingency reserve activation processes. 
Figure 1: Probabilistic Display Concept

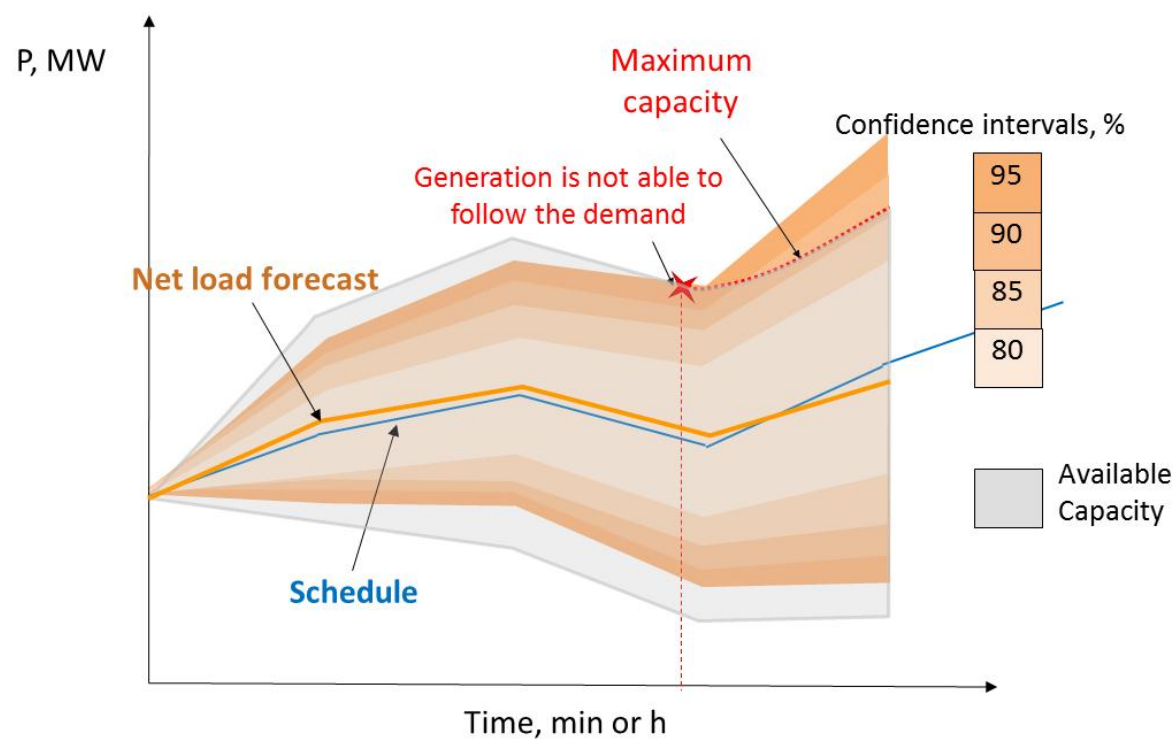

- Create an integrated tool that consolidates the above-mentioned continuous and discrete random factors, contributing to the overall uncertainty, to evaluate look-ahead, worstcase balancing generation requirements (performance envelopes) in terms of the required capacity, ramping capability, and ramp duration.

- Build a methodology and procedures for self-validation of the predicted performance envelope for each look-ahead interval.

- Develop visualization displays to communicate information about the expected ramps and their uncertainty ranges.

- Develop a framework of the tool integration into the California Independent System Operator's (CAISO's) EMS and market systems

- Use actual CAISO data to perform simulation.

The following results have been achieved in this work:

Innovative methodology and software tools have been developed that are capable of evaluating future generation requirements, including the required capacity, ramping capability, and ramp duration capability (performance envelope) in view of uncertainties caused by wind and solar generation and load forecast errors. The approach includes three stages: 1) forecast and actual data acquisition, 2) statistical analysis of retrospective information, and 3) prediction of future grid balancing requirements for specified time horizons and confidence intervals Assessment of the capacity and ramping requirements is performed using a specially developed probabilistic algorithm based on a histogram analysis incorporating all sources of uncertainty and parameters of a continuous and discrete nature (Figure 2).

- A "flying brick" method has been developed to assess the look-ahead worst-case performance envelope requirement to be able to enable the system to accommodate the uncertainties with certain specified degree of confidence (Figure 3). The "flying brick" 
concept is to simultaneously include the ramp rate, ramp duration, and capacity requirements directly in the balancing process.

Figure 2: Probabilistic Assessment Methodology Concept

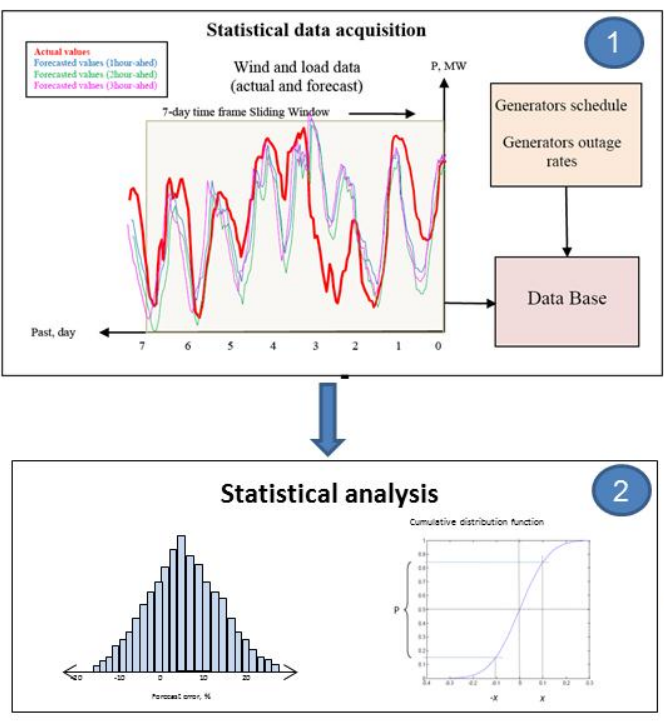

1. Data acquisition

2. Uncertainty assessment through statistical analysis

3. Prediction of future grid balancing requirements for specified time horizons and confidence levels

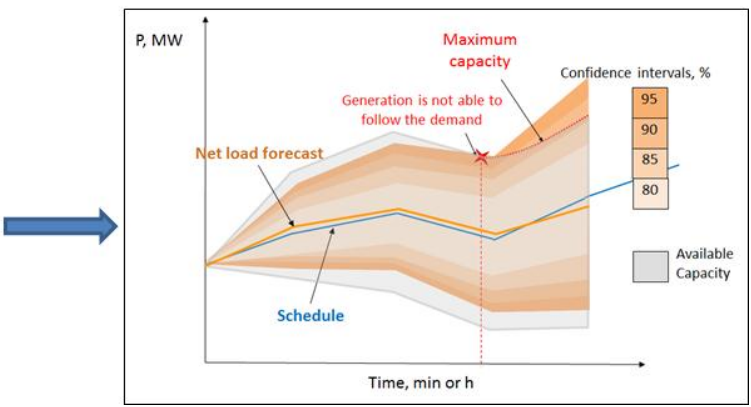

Figure 3: "Flying Brick" Method

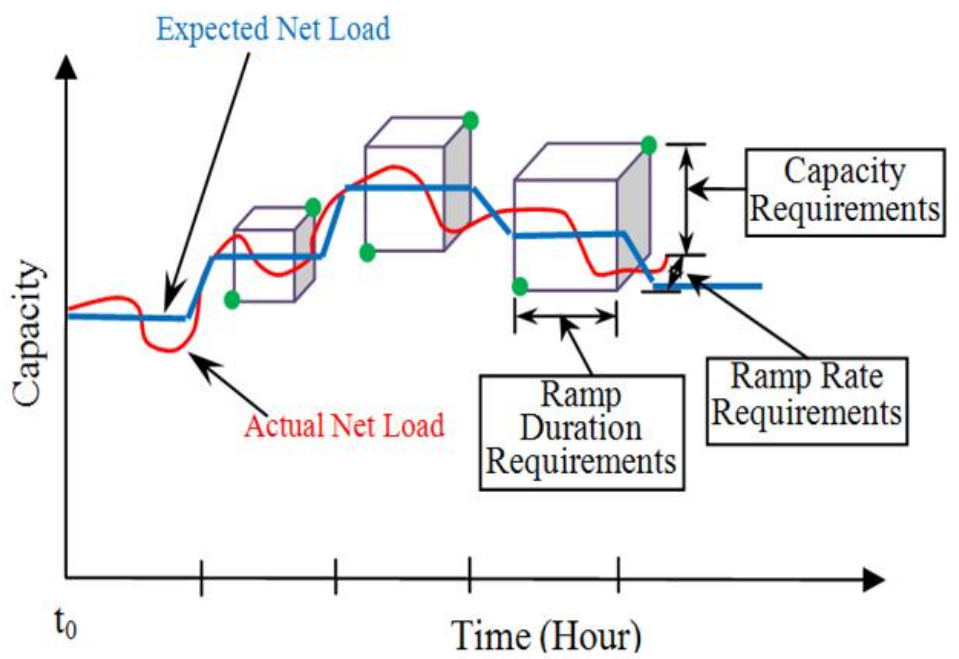

- A self-validation approach has been proposed. The purpose of the self-validation algorithm is to verify that the uncertainty ranges predicted based on retrospective information are valid for the future dispatch intervals. 
- An industrial software tool has been developed and tested. An example of software tool graphical user interface (GUI) is presented in Figure 4.

- Simulations using actual data provided by this project's CAISO engineering support team have been carried out. Simulation results have shown that the proposed methodology is quite accurate and efficient.

Figure 4: Software Tool GUI

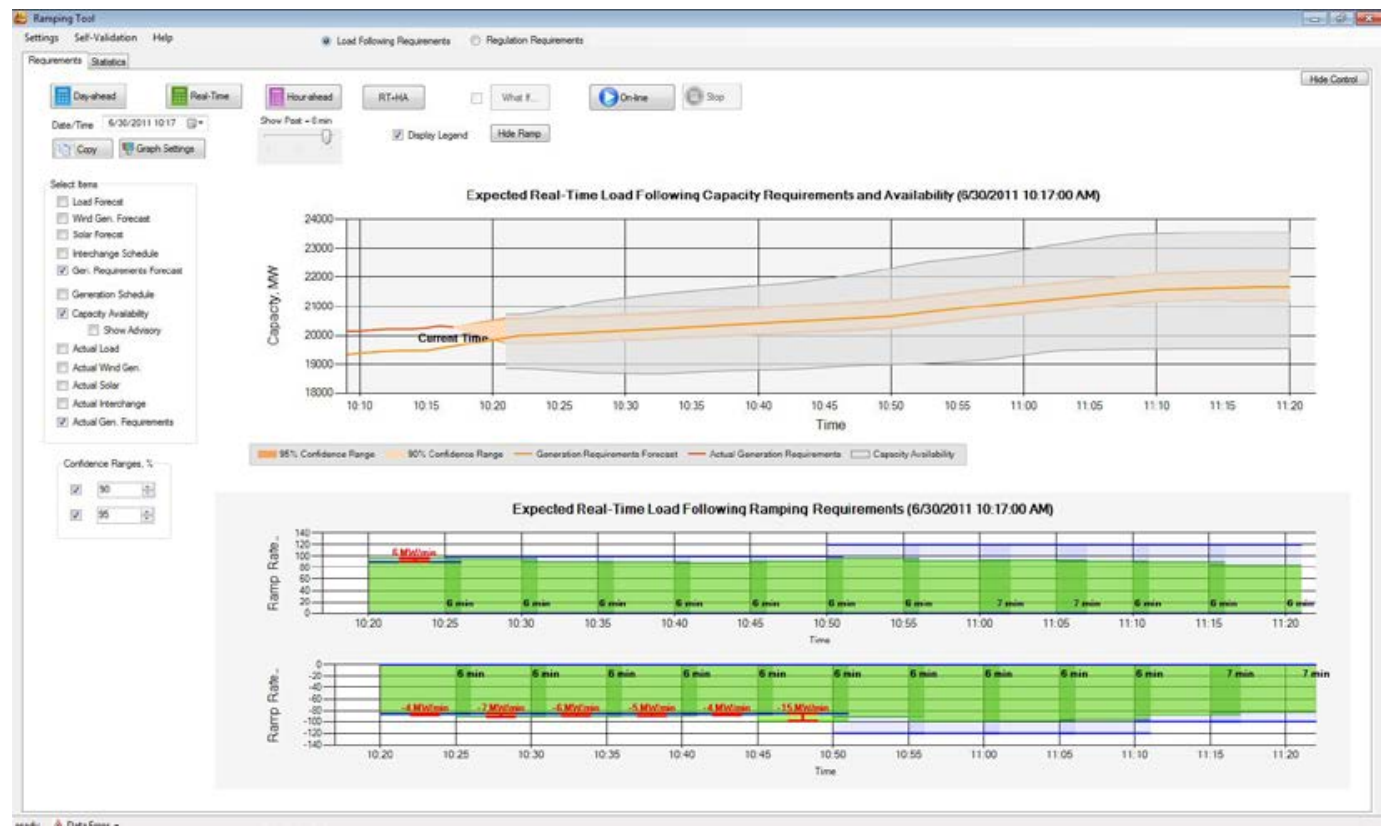

It has been found that the tool is capable of predicting intra-hour deficiency in generation capability. This deficiency of balancing resources can cause price spikes in the real-time market. As an example, one can see in

Figure 5 that a greater than $300 \mathrm{MW}$ deficiency in the system generation capability is predicted in the 10- to 25-min look-ahead period (red error bars). Figure 6 shows the CAISO's real-time market price information. Price spikes occurred at CAISO market at the same time at which generation capability deficiency was predicted by the tool.

- The concept of probabilistic tool integration into EMS has been developed. The concept includes three levels of integration: a passive level, an active level, and a proactive level. The passive integration level implies integration of wind forecast information and its visualization without introducing any changes to the EMS algorithms. On the active level, the unit commitment (UC) and economic dispatch (ED) procedures are repeated several times for every dispatch interval to determine whether the system can meet the limits of generation requirements caused by uncertainties for a certain confidence level (Figure 7). The system "breaking points" are communicated to the user. The proactive level requires some modifications of the $\mathrm{UC}$ and ED algorithms in order to directly 
incorporate uncertainties into these procedures (Figure 7). In this case, the generation units will be committed and dispatched, so that these uncertainties would not create "breaking points."

Figure 5: Real-Time Capacity Requirements Prediction (6/24/11 16:15)

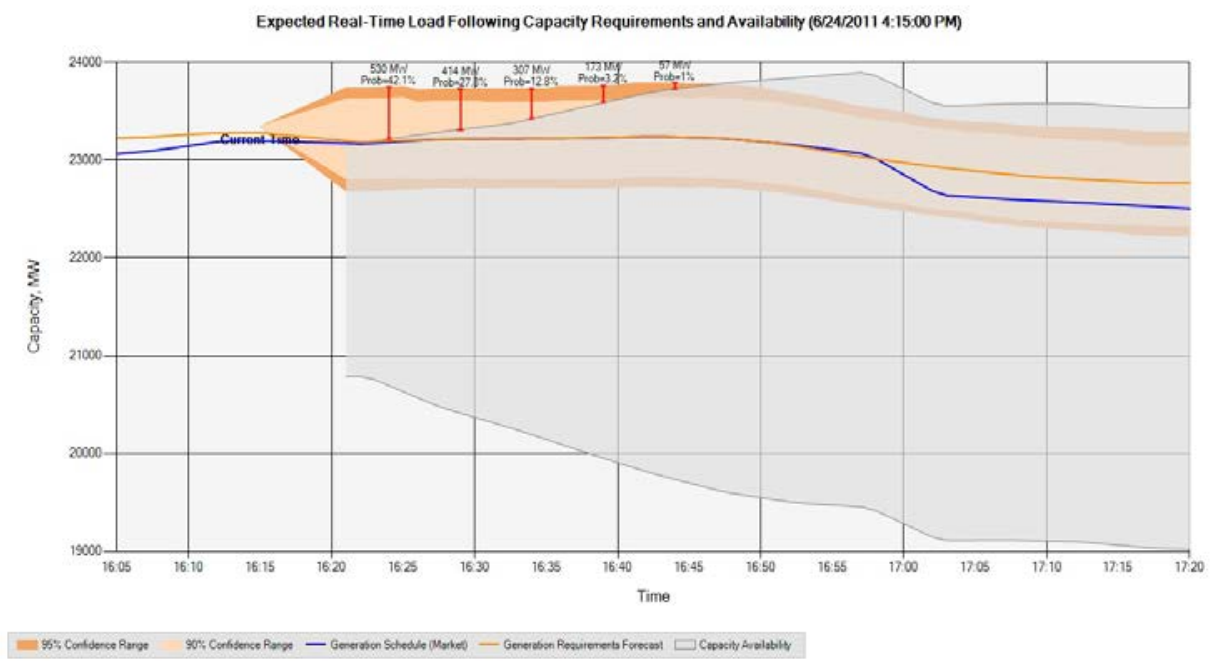

Figure 6: CAISO Real-Time Price (6/24/11) ${ }^{1}$

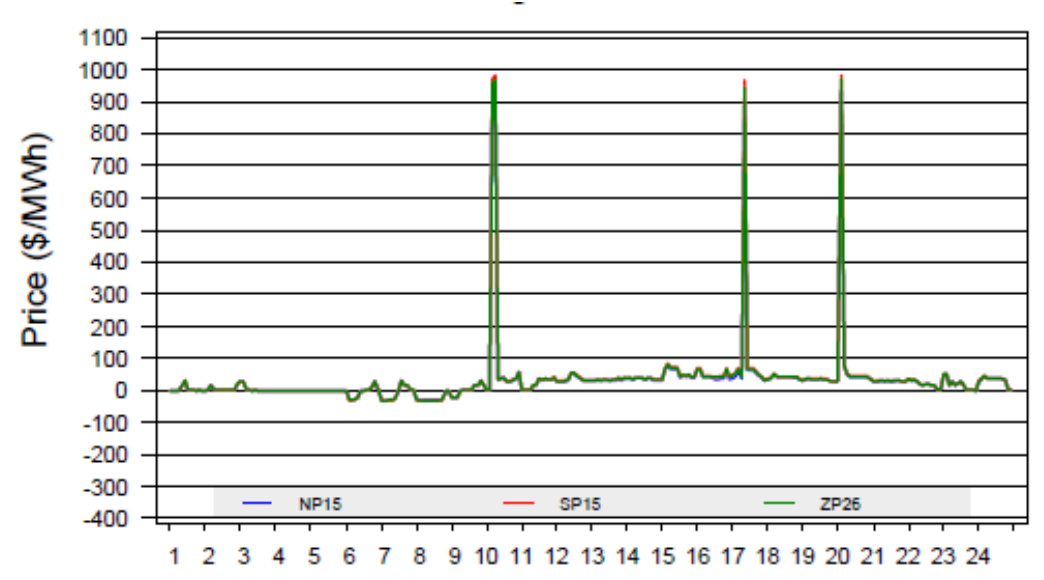

${ }^{1}$ Available at http://www.caiso.com/Documents/June\%202011/DailyMarketWatch RealTime Jun 24 2011.pdf 
Figure 7: "Active" and "Proactive" Integration Approaches

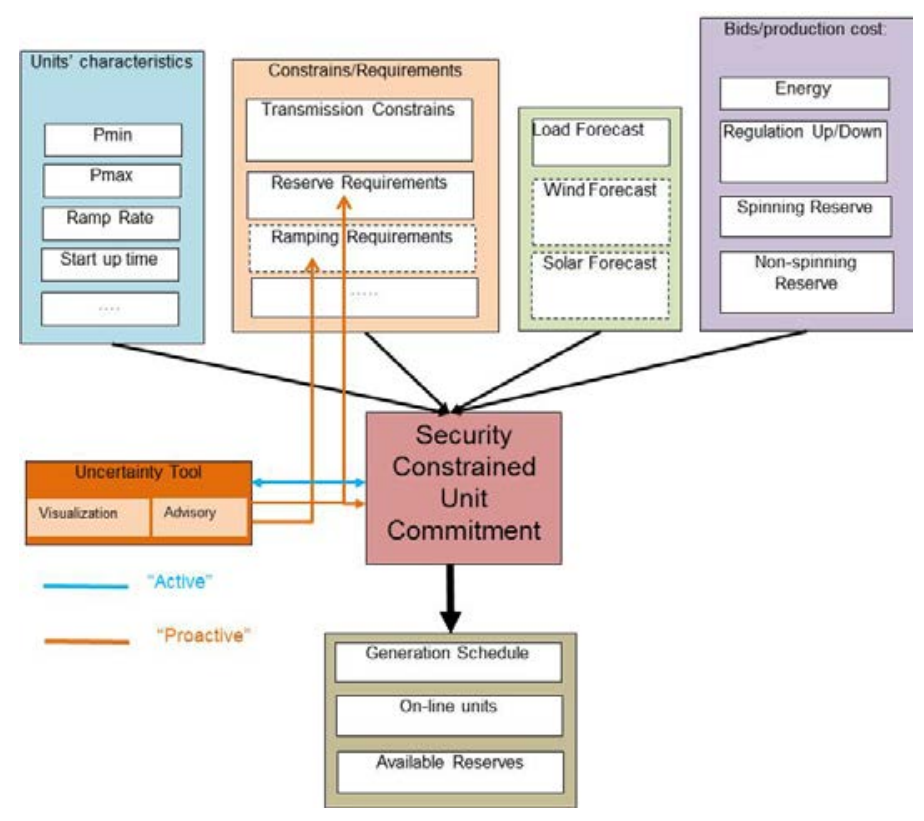

The following recommendations for the next phase have been made:

- Integration of the tool with the wind (solar) forecast service providers' information to improve the accuracy of the balancing requirements prediction.

- Continue development and implementation of proactive integration methodology to incorporate the tool into CAISO market and unit commitment procedures.

- Development and implementation of a new generation of statistical methods to address non-stationary characteristics of forecast errors. Further improve the accuracy and robustness of the tool.

- Probabilistic modeling of uninstructed deviation of generating units

- Developing a decision support system using the PNNL tool to help dispatchers address potential issues of the system balancing process.

- Incorporating consideration of the new Balancing Authority ACE (Area Control Error) Limit (BAAL) standard into balancing requirements assessment methodology.

- Addressing data interpretation and quality issues to enhance robustness of the tool.

The planned commercialization activities include a wide dissemination and technology transfer effort with the ultimate objective to install the tool in several control centers in California. Among these activities, the highest priority tasks will include a proactive integration of the software tool in the CAISO control center, its comprehensive testing and final refinements. The results of this effort will create a platform for a wider reach in California by serving as an example for other control areas and utilities in this state. It is expected that several more organizations will become interested in installing the tool in their control centers. In parallel with the California activities, the results of this project will be widely distributed via Web 
seminars organized for the industry and by presenting them at the industry forums, including Utility Wind Integration Group, Western Electricity Coordinating Council groups, North American Electric Reliability Corporation subcommittees, and conferences. It is expected that through these activities (supported by the U.S. Department of Energy), the impact of this project will be extended from the statewide level to the nationwide level. 


\section{CHAPTER 1: Introduction}

\section{Background}

The work described in this report was performed by Pacific Northwest National Laboratory (PNNL) and funded by the California Energy Commission (CEC).

Because conventional generators need time to be committed and dispatched to a desired megawatt (MW) level, the scheduling and load following processes use load and wind power production forecasts to achieve future balance between the conventional generation and energy storage on the one side, and system load, intermittent resources (such as wind and solar generation) and scheduled interchange on the other side. The power system balancing process, which includes the scheduling, real-time dispatch (load following) and regulation processes, is traditionally based on deterministic models.

Uncertainties in forecasting the output of intermittent resources, such as wind and solar generation, as well as system loads are not reflected in existing energy management systems (EMS) and tools for generation commitment, dispatch and market operation. With the growing penetration of intermittent resources, these uncertainties could result in significant unexpected load-following and dispatch problems, and pose serious risks to control and operation performance characteristics as well as the reliability of a power grid. Without knowing the risks posed by the uncertainties, the system operators have limited means to weigh the likelihood of occurrence and the magnitude of problems to mitigate adverse impacts caused by them. Some important questions need to be addressed in counteracting the impact of uncertainties; for instance, whether and when one should start more units to balance against possible fast ramps in the future over a given time horizon.

Furthermore, these uncertainties could require procuring additional costly balancing services. Major unexpected variations in wind power that are unfavorably combined with load forecast errors and forced generator outages could cause significant power mismatches, which could be essentially unmanageable without knowing these variations in advance.

Because the actual load and intermittent generation can deviate from the forecasts, it becomes increasingly important to assess with increasing penetration of renewable resources whether the system would actually be able to meet the conventional generation requirements within the look-ahead horizon, what additional balancing efforts would be needed as we approach real time, and what additional costs would be incurred by those needs.

In order to improve the system control performance characteristics, maintain system reliability, and minimize expenses related to the system balancing functions, it becomes necessary to incorporate the projected uncertainty ranges into the scheduling, load following, and, to some extent, into the regulation processes. This need has been realized already, and some wind 
forecast service providers offer the uncertainty information for their forecasts. Works are in place to develop methodologies and tools to incorporate these uncertainties into power system operations.

Some wind forecast service providers offer uncertainty information for their forecasts. For instance, AWS Truepower (Zack 2006) and 3TIER (Lerner et al. 2009) companies developed wind power generation forecasting tools with built-in capability to assess wind generation uncertainty. Similar tools have been developed in Europe. In the context of a European Union project, ANEMOS, a tool for on-line wind generation uncertainty estimation based on adaptive re-sampling or quantile regression has been developed (Kariniotakis 2008). A German company, Energy and Meteo Systems, developed a tool for wind generation forecasting, assessing the uncertainty ranges associated with wind forecast, and predicting extreme ramping events (Energy \& Meteo Systems 2007). Pinson et al. (2006) discusses a wind generation interval forecast approach using the quantile method. Luig et al. (2001) used statistical analysis based on standard deviation to predict wind generation forecast errors. Work is underway to incorporate these uncertainties into power system operations (Kehler et al. 2010; Maggio et al. 2010). In Constantinescu et al. (2011), Wang et al. (2008), and Ummels et al. (2007), wind power generation is taken into account in the unit commitment problem.

Unfortunately, in many cases, these efforts are limited to wind generation uncertainties only, and ignore the fact that there are additional sources of uncertainty such as system loads and forced generation outages. Most of the works are considering only the uncertainty ranges for the MW imbalances, and do not address additional essential characteristics such as ramps and ramp duration uncertainties.

It is very important to address the uncertainty problem comprehensively, by including all sources of uncertainty (load, intermittent generation, generators' forced outages, etc.) into consideration. All aspects of uncertainty such as the imbalance size (which is the same as capacity needed to mitigate the imbalance) and generation ramping requirement must be taken into account. The latter unique features make this work a significant step forward toward the objective of incorporating of wind, solar, load, and other uncertainties into power system operations.

In this project, the uncertainties associated with wind power generation forecasting, load demand forecasting, and generation supply interruptions caused by forced outages are taken into account in the evaluation of uncertainty ranges for the required generation performance envelope, including balancing capacity, ramping capability and ramp duration. A probabilistic algorithm, based on the proposed histogram analysis to assess the capacity and ramping requirements, is presented. Simulation was performed using California Independent System Operator's (ISO')s system model and data. This report presents these simulation results confirming the validity and efficiency of the proposed solutions.

A probabilistic software tool capable of determining the impact of wind, solar, load and generation uncertainties on the power grid is currently under development. In the research, an 
assessment of generation capacity requirements means evaluation of uncertainty ranges of generation requirements in order to meet the power system balance.

\section{Goals of This Project}

The objective of this project is to develop a tool to estimate California Independent System Operator's (CAISO's) upward and downward balancing requirements in terms of its capacity, ramp rate and ramp duration for a user-specified time horizon (several hours ahead or the next day). Based on a scientific approach that uses a specified level of confidence, the estimate will indicate balancing requirements without compromising reliability or compliance with mandatory control performance standards.

This report discusses the following developments:

- a probabilistic model to evaluate uncertainties of the wind and load forecast errors and to provide rapid (every 5 minutes) look-ahead (up to 5-8 hours ahead) assessments of their uncertainty ranges

- similar models to evaluate uncertainties caused by random generator forced outages

- an integrated tool that consolidates the abovementioned continuous and discrete random factors contributing to the overall uncertainty to evaluate look-ahead worst-case balancing generation requirements (performance envelopes) in terms of the required capacity, ramping capability, and ramp duration

- a methodology and procedures for self-validation of the predicted performance envelope for each look-ahead interval

- visualization displays to communicate information about the expected ramps and their uncertainty ranges

- use of the actual CAISO data to perform simulation

- review of the actual dispatch scheduling processes in CAISO

The proposed approach to evaluate the uncertainty ranges for a required generation performance envelope, including the balancing capacity, ramping capability and ramp duration, consists of the following three stages (Figure 8):

1. The first stage deals with acquiring statistical data. Retrospective information for a userspecified moving window (e.g., for one month), such as forecasted system load and its actual values, wind and solar generation forecasts and their actual values, as well as generation schedules, are needed to perform the proposed statistical analysis and to build a projection of the balancing requirements into the future.

2. The second stage is a statistical analysis of the retrospective information acquired at the first stage. It consists of the following parts:

- capacity requirements analysis based on an empirical statistical analysis of forecast errors 
- ramping requirements analysis based on the "swinging door" algorithm

- generation forced outage analysis based on the Markov chain reliability model

Figure 8: Methodology Concept

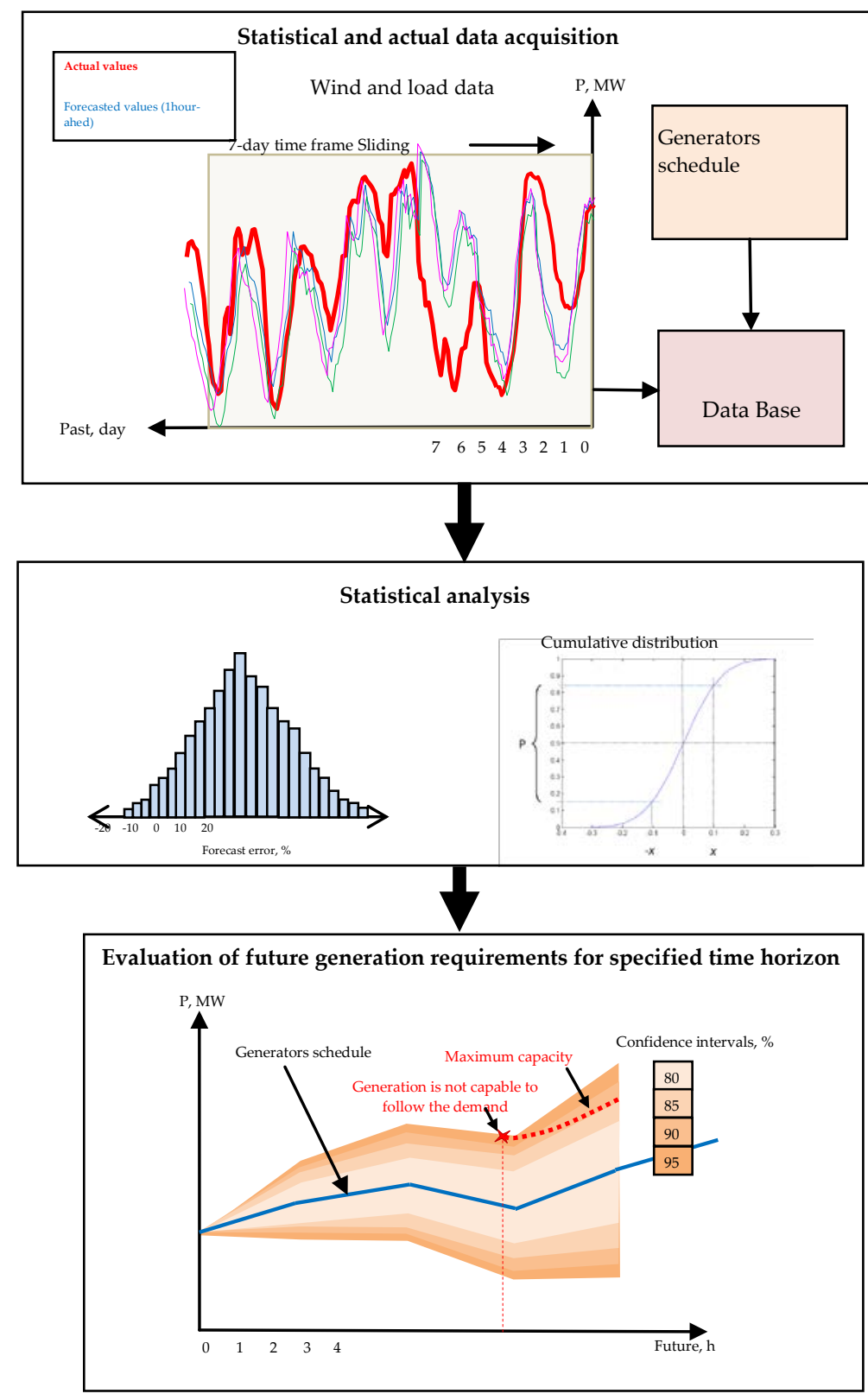

3. The third stage is an evaluation of future generation requirements for specified time horizons, e.g., 5 or 8 hours ahead. Examples of generation requirements that can be evaluated are regulation and load-following capacity requirements, ramping requirements, contingency reserve requirements, etc., for different confidence levels such as $80,85,90$, and 
95\% (as shown in Figure 8). These requirements can be compared against the actual generation capability of generators that are currently or will be online within the look-ahead horizon and that are performing relevant services. If the actual generation capability is not matching the requirements, a warning will be issued to system operators. This would constitute a "passive" integration of wind-related uncertainties into the system operations. In a proactive approach, the look-ahead generation requirement information will be fed back into the generation commitment and dispatch procedures in order to modify them and to make sure that the generators are committed on time and dispatched to be able to meet the capacity, ramping and ramp duration requirements with a certain level of confidence for the entire look-ahead period.

\section{Report Organization}

The report is organized as follows. Chapter 2 discusses evaluation of uncertainties associated with the system load forecast and wind generation forecast using different statistical methods. Chapter 3 reviews the current operating processes and schedules followed by the CAISO. Chapter 4 presents software prototype design. Chapter 5 provides testing and simulation results. Chapter 6 provides conclusions and future work; a list of references is provided in Chapter 7 and a glossary in Chapter 8.

Appendices are also included that provide additional information on the ramping capability analysis methodology and forced-outage model. 


\section{CHAPTER 2: \\ Load and Wind/Solar Generation Uncertainty Evaluation}

This section describes an innovative methodology that is capable of evaluating future generation requirements including the required capacity, ramping capability, and ramp duration capability (these characteristics form the so-called performance envelope). The methodology incorporates uncertainties caused by wind generation and load forecast errors as well as (potentially) uninstructed generation deviations of conventional generation. These tools meet the industry need in a more robust (that is, more reliable for a range of possible future operating conditions) assessment of the balancing reserves required in a control area.

The previous works discussed in the introduction (Zack 2006; Lerner et al. 2009; Kariniotakis 2008; Energy \& Meteo Systems 2007; Pinson et al. 2006; Luig et al. 2001; Kehler et al. 2010; Maggio et al. 2010; Constantinescu et al. 2011; Wang et al. 2008; Ummels et al. 2007) address only a single source of uncertainty: the one related to wind generation. Because the influence of the other sources of uncertainty is not reflected in the assessment, the resulting confidence intervals are of limited value to the system operators. Unlike these existing approaches, the methodology developed in this report addresses all sources of uncertainty, including the uncertainties surrounding the load forecasts, uncertainties associated with the forced generator outages, and uncertainties caused by forced generation outages (see Appendix B).

A "flying brick" method was developed in this study to assess the look-ahead worst-case performance envelope requirements and to be able to make sure the system has the capability to balance against the uncertainties with a certain specified degree of confidence. The "flying brick" concept is to include the ramp rate, ramp duration, and capacity requirements simultaneously and directly in the balancing process, and then look for the worst combinations of these parameters located along the vertices' trajectories of the "flying brick."

\section{Generation Reserves}

According to the Western Electricity Coordinating Council (WECC), standard power systems are required to maintain the following types of reserves (WECC 2007):

Operating reserve: the generation capacity above the amount needed to supply firm system demand that is required to provide for regulation, to balance against the load forecasting error and equipment forced and scheduled outages, and to maintain local area reliability. It consists of spinning reserve and non-spinning reserve.

Spinning reserve: unloaded generation that is synchronized, automatically responsive to frequency deviations, and ready to serve an additional demand. It consists of regulating reserve and contingency reserve. 
Non-spinning reserve: 1 . The generating reserve that is not connected to the system but capable of serving the demand within a specified time from its activation. 2. Loads or exports that can be removed from the system in a specified time.

Regulating reserve: an amount of reserve responsive to automatic generation control (AGC), which is sufficient to provide normal regulating margin.

Contingency reserve - The capacity available to be deployed by a balancing authority (BA) to meet the North American Electric Reliability Corporation (NERC) and WECC contingency reserve requirements. Increasing penetration of wind and solar generation leads to growing uncertainties in the reserve requirements.

In the study, the term "assessment of generation capacity requirements" refers to the evaluation of uncertainty ranges for generation requirements needed to achieve power system balance. These uncertainty ranges define intervals within which the future generation requirement is expected to lie with a specified level of confidence.

Uncertainties associated with wind and solar intermittency, electrical load variability, and unexpected generation outages are considered in this report. These uncertainties affect the load following needs as well as regulating and contingency reserve requirements. Details regarding the load and wind/solar generation uncertainties are given in this section; description of the generation forced outage model is presented in Appendix B. In general, the generation capacity allocation is performed by the unit commitment (UC) process, as shown in Figure 9 (WSCC 1998).

To integrate the probabilistic tool into an EMS, it is necessary to take into account the operating practices of the given power system. In Chapter 3, details of the operating practices at CAISO are presented.

Figure 9: Allocation of Generation Unit Capacity

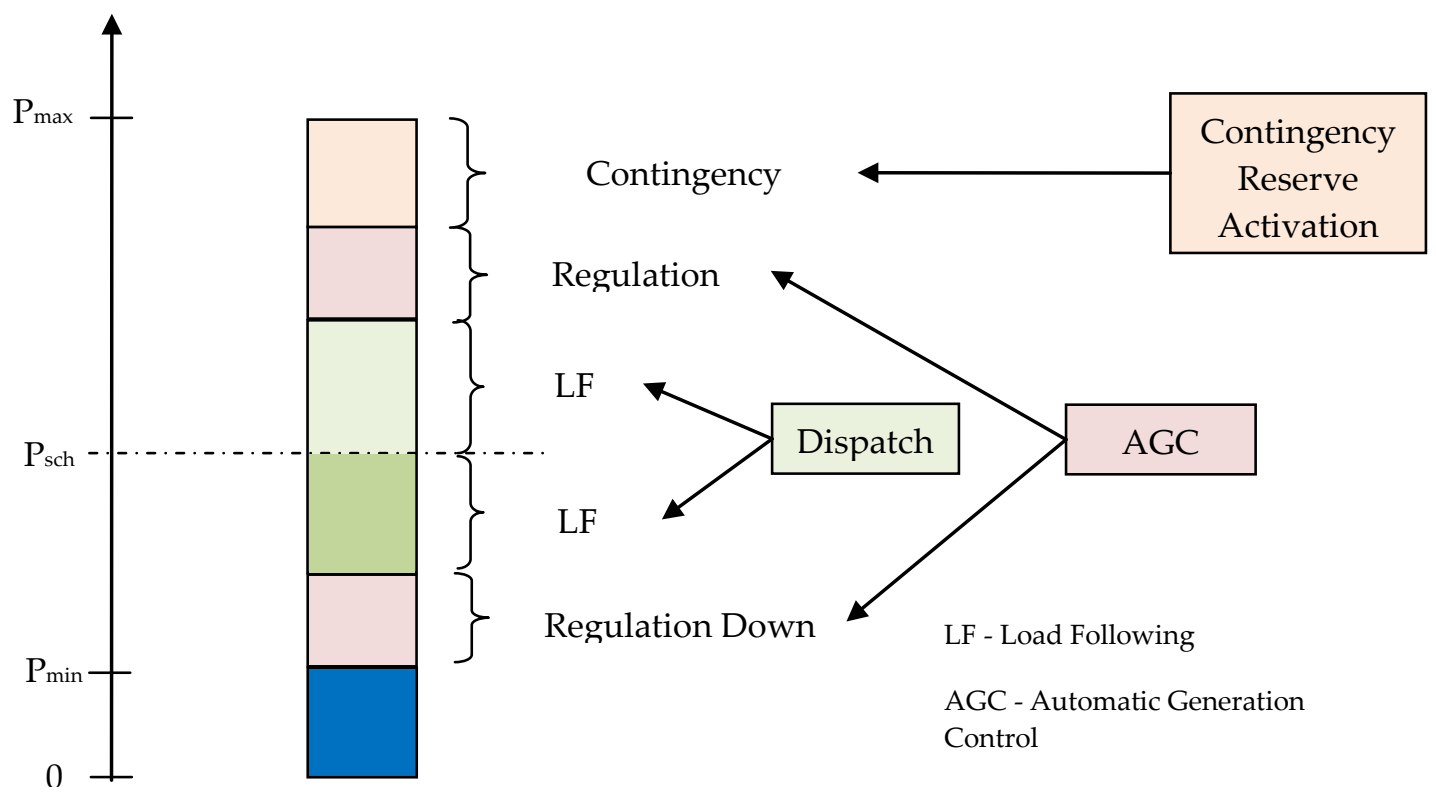




\section{Load Forecast Uncertainty}

The uncertainty associated with the load forecast is one of the most influential factors influencing the overall uncertainty. In Figure 10, the load forecast uncertainty for one of the balancing authorities is shown. The solid blue curve in Figure 10 (a) shows the hourly average demand over one month, while the red curve shows the day-ahead load forecast for the same time period. The load forecast error is presented in Figure 10 (b). One can see that day-ahead load forecast has an error of upto about $\pm 8 \%$. What is also important is the fact that the system load is normally more significant than wind or solar generation, so that even if the load forecast is more accurate than the forecast for the intermittent resources (in terms of the percentage error), the MW values of the errors can be quite comparable.

Figure 10: Load Fluctuation and Uncertainty: a) Day-Ahead Load Forecast vs. Actual Load; b) Load Forecast Error

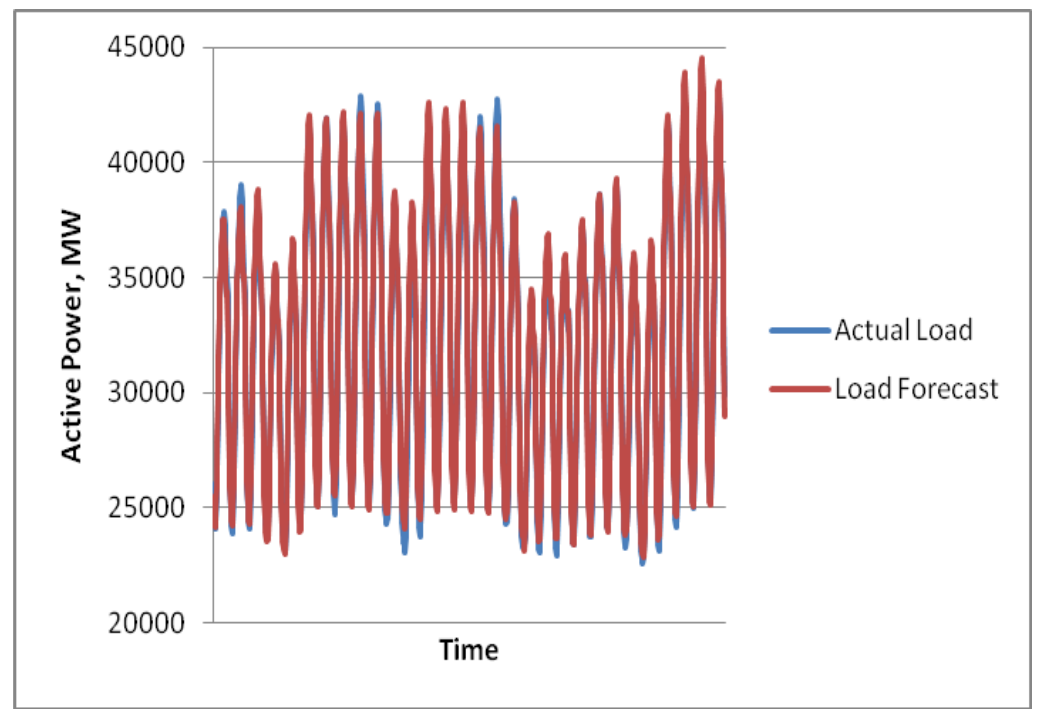

a)

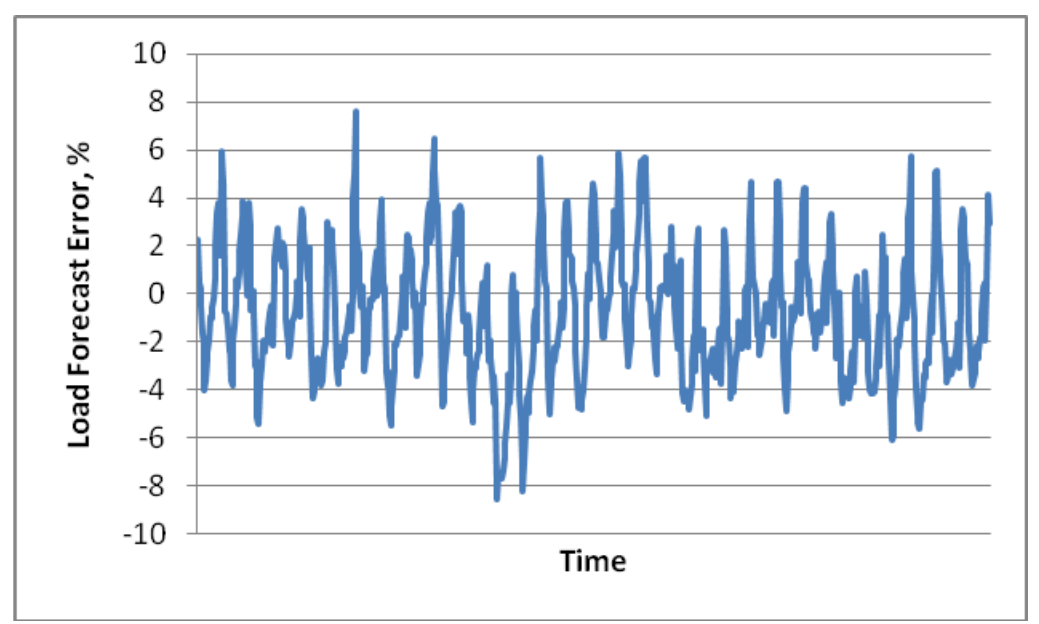

b) 


\section{Net Load Uncertainty}

Wind and solar generation and power system load demand have a number of similar features:

- Wind/solar generation and most of the load are non-dispatchable resources.

- They both have cycling behavior.

- They both depend on the weather conditions.

- They deviate from the forecast, etc.

Actually, wind generation has more in common with electrical load than with traditional (dispatchable) generation; therefore, wind generation can be considered as a negative load. At the same time, electrical load and wind/solar generation cannot be considered as independent statistical variables. The correlation between load and wind generation forecast errors is shown in (Constantinescu et al. 2011; Wang et al. 2008). To address this issue, the net load concept is commonly used in wind integration studies to assess the impact of load and wind generation variability on the power system operation. The net load has the following definition: net load is total electrical load minus total wind generation output, minus total solar generation output, plus the interchange.

\section{Statistical Methods to Evaluate the Forecast Uncertainty}

There are different approaches that can be used for the uncertainty analysis of the forecast errors. In this work, we analyzed two methods in terms of their applicability for the purpose of this project: distribution fitting and empirical probability.

\section{Distribution Fitting Approach}

Probability distributions are based on assumptions about a specific standard form of random variables; for example, normal, uniform or Poisson distributions. Based on the standard distributions and selected set of its parameters, they assign probability to the event that the random variable takes on a specific, discrete value, or falls within a specified range of continuous values (MathWorks ${ }^{\circledR} 2010$ ).

Selecting a distribution model means choosing a standard probability distribution and then adjusting its parameters to fit the data (MathWorks ${ }^{\circledR}$ 2010). For example, in (CAISO 2007) it is assumed that the load and wind forecast errors are described by the truncated normal distribution (TND).

The probability density function (PDF) of the truncated normal distribution is:

$$
P D F_{T N D}(x ; \mu, \sigma, a, b)=\frac{\frac{1}{\sigma} P D F_{N}\left(\frac{x-\mu}{\sigma}\right)}{C D F_{N}\left(\frac{b-\mu}{\sigma}\right)-C D F_{N}\left(\frac{a-\mu}{\sigma}\right)}
$$

where

$\mu=$ the mean value of the non-truncated normal distribution;

$\sigma=$ the standard deviation of the non-truncated normal distribution; 


$$
\begin{aligned}
& a, b=\text { upper and lower limits of the non-truncated normal } \\
& \text { distribution; } \\
& x \in(a, b),-\infty \leq a<b \leq \infty \text {; } \\
& \mathrm{PDF}_{N}(x ; \mu, \sigma)=\frac{1}{\sigma \sqrt{2 \pi}} e^{-\frac{(x-\mu)^{2}}{2 \sigma^{2}}}=\text { the probability density function of the standard normal } \\
& \sqrt{ } \text { distribution, and } \\
& C D F_{N}(\cdot)=\text { the cumulative distribution function }(\mathrm{CDF}) \text { of the } \\
& \text { standard normal distribution. }
\end{aligned}
$$

The cumulative distribution function of the truncated normal distribution is:

$$
C D F_{T N D}(x ; \mu, \sigma, a, b)=\frac{\operatorname{CDF}_{N}\left(\frac{x-\mu}{\sigma}\right)-C D F_{N}\left(\frac{a-\mu}{\sigma}\right)}{C D F_{N}\left(\frac{b-\mu}{\sigma}\right)-C D F_{N}\left(\frac{a-\mu}{\sigma}\right)}, \quad a \leq x \leq b
$$

An example of the load forecast error distribution is presented in Figure 11. The blue bars represent the histogram of the real load forecast error. The red curve depicts the TND of the load forecast error.

Figure 11: Load Forecast Error Histogram

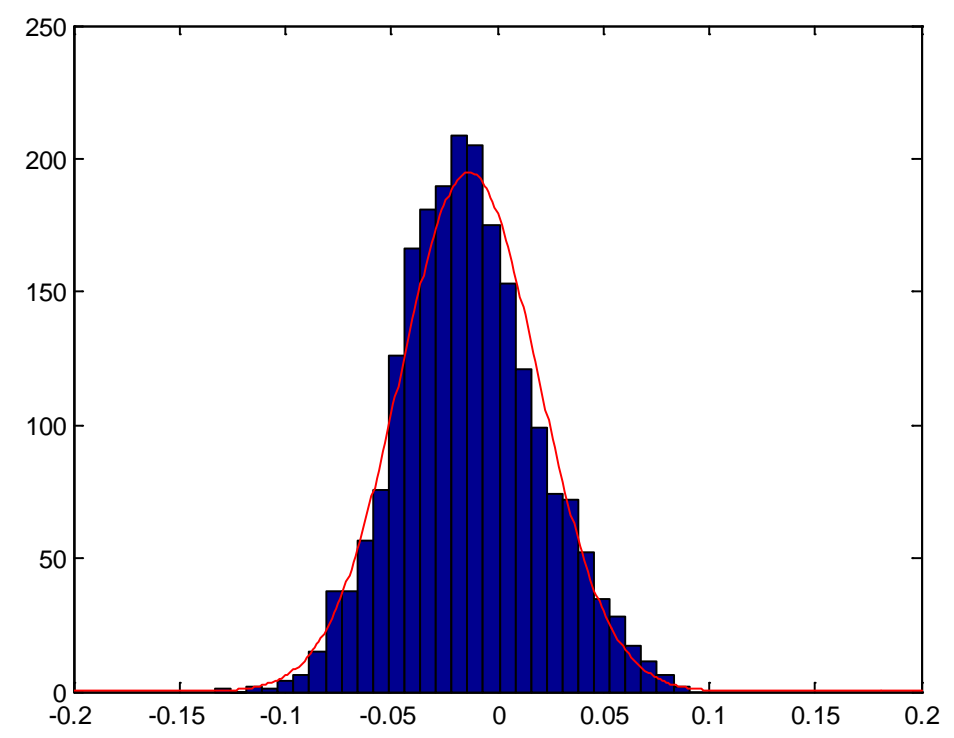

\section{Empirical Probability Approach}

When data do not follow a standard probability distribution, nonparametric models based on empirical probability distributions are more appropriate. These models have the advantage of 
making no assumptions about the form of the underlying distribution, so no parameter estimates are needed (MathWorks ${ }^{\circledR}$ 2010). The idea behind building the empirical Cumulative Distribution Function (CDF) is relatively simple. This is a function that assigns probability $1 / n$ to each of $n$ observations in the analyzed dataset. The CDF for a given value $x$ of a parameter is calculated by adding all probabilities for the samples that have values smaller than $x$. Its graph has a stair-like appearance. An example of an empirical distribution (net load forecast error distribution and empirical CDF) are presented in Figure 12.

Figure 12: Net Load Forecast Error Distribution: a) Histogram; b) Empirical CDF

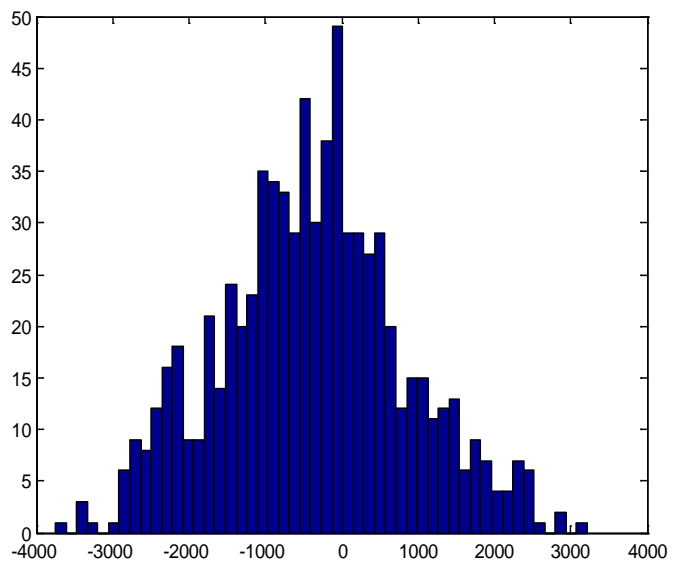

a)

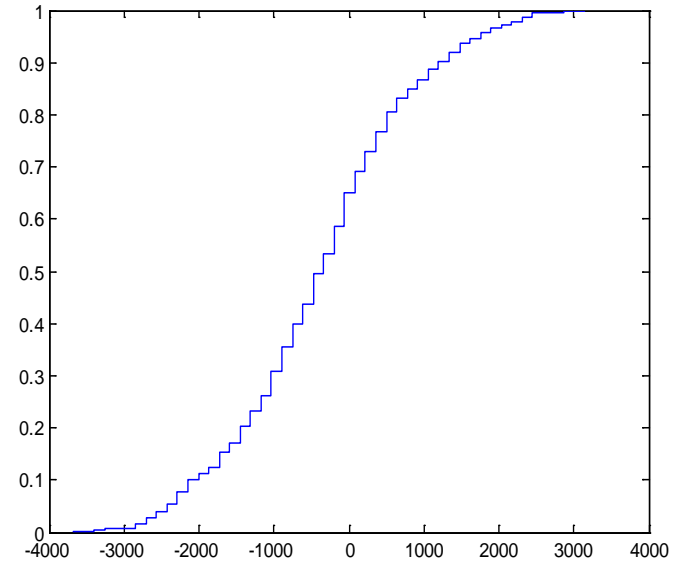

b)

\section{Assessment of the Generation Capacity Uncertainty}

A statistical approach based on the time-varying empirical probability density function (PDF) is used in the study to determine the combined uncertainty ranges of the wind/solar and load forecast errors, as well as the effect of the forced generation outages. In this section, we only consider the wind and load forecast error uncertainties. The solar forecast error can be included into the consideration in the same way as the wind forecast error. The methodology to incorporate the generator forced-outages uncertainty will be given in the following sections.

In our approach, wind and load forecast errors are summed together for each dispatch interval in the past within a sliding window. The sliding window size is selected to collect sufficient statistical information regarding the forecast errors. The information can be accumulated separately for each forecast horizon; for instance, for the hour-ahead forecast, two hours ahead forecast, and so on. Based on the collected statistics, the approach evaluates the percentile intervals (also called confidence intervals or uncertainty ranges) for each forecast horizon and different level of confidence. These intervals are assumed to be the same in the future dispatch interval; that is, for the next hour, the hour after that, and so on.

An example, based on data from a real power system, of the statistical characteristics of wind generation forecast for different look-ahead dispatch intervals $(1,2,3,4$, and 5 hours ahead) is 
presented in Figure 13. Figure 13 (a) shows the empirical PDF and Figure 13 (b) shows the empirical CDF.

The uncertainty range defines an interval within which a random parameter is expected to lie with a specified level of confidence. To determine an uncertainty range, it is necessary to find two solutions of the inverse CDF function corresponding to the desired percentiles on both ends of the distribution. The definition of the inverse $\mathrm{CDF}$ is the following:

For a strictly increasing and continuous CDF, for a given value $p$ of the probability, the inverse CDF function $C D F^{-1}(p), p \in[0,1]$ is the unique real number $x$ such that $C D F(x)=p$. The inverse of the CDF is called the quantile function. An evaluation of the quantile functions often involves special numerical methods.

Our task is to find the forecast error range $x_{1} \ldots x_{2}$ to the given level of confidence $p$

$$
C D F\left(x_{2}\right)-C D F\left(x_{1}\right)=p\left(x_{1} \leq X \leq x_{2}\right)=\int_{x_{1}}^{x_{2}} P D F(x) d x
$$

Inverse CDF functions for wind generation forecast errors for different look-ahead periods are presented in Figure 14. The uncertainty ranges are evaluated at a 95\% confidence level. The 95\% uncertainty range corresponds to the 2.5 to 97.5 percentile range of the distribution reflecting the uncertainty (Figure 14). It is clear that the size of uncertainty ranges depends on the lookahead time. It can be seen from Figure 14 that for the longer look-ahead periods, the uncertainty range becomes larger.

Figure 13: Statistical Characteristics of Wind Generation Forecast for Different Look-Ahead Periods: a) PDF; b) Empirical CDF

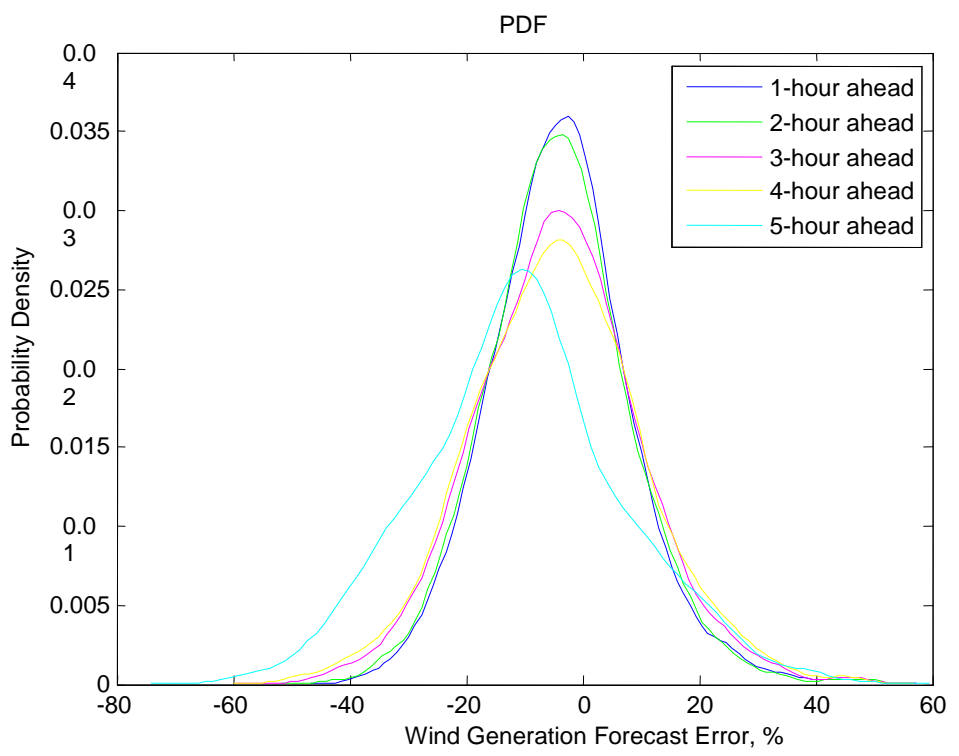




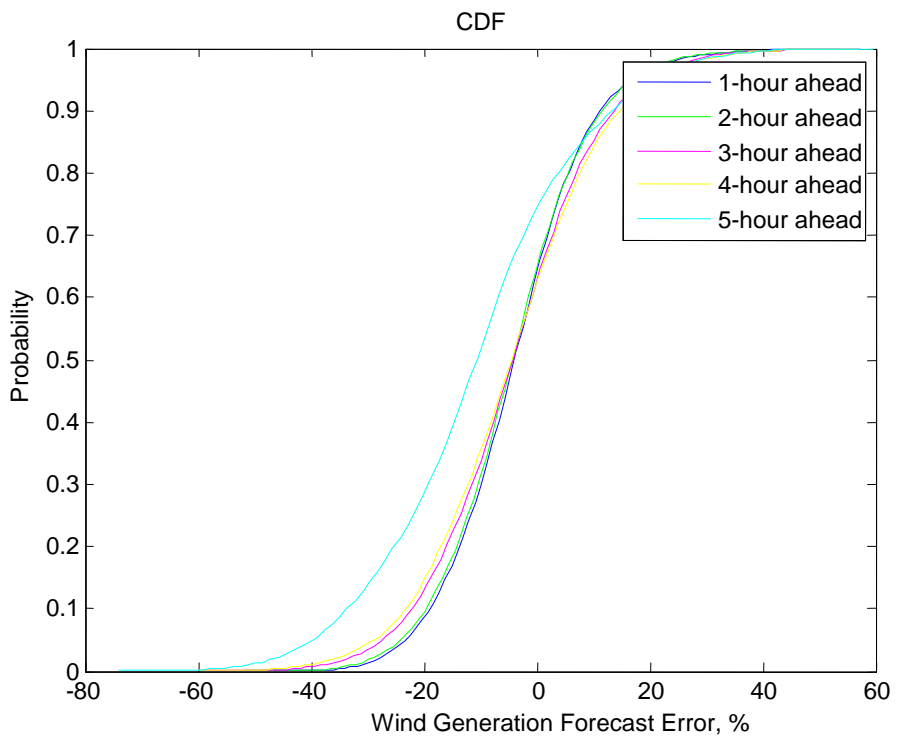

b) 
Figure 14: Wind Generation Forecast Inverse CDFs for Different Look-Ahead Intervals and 95\% Uncertainty Ranges

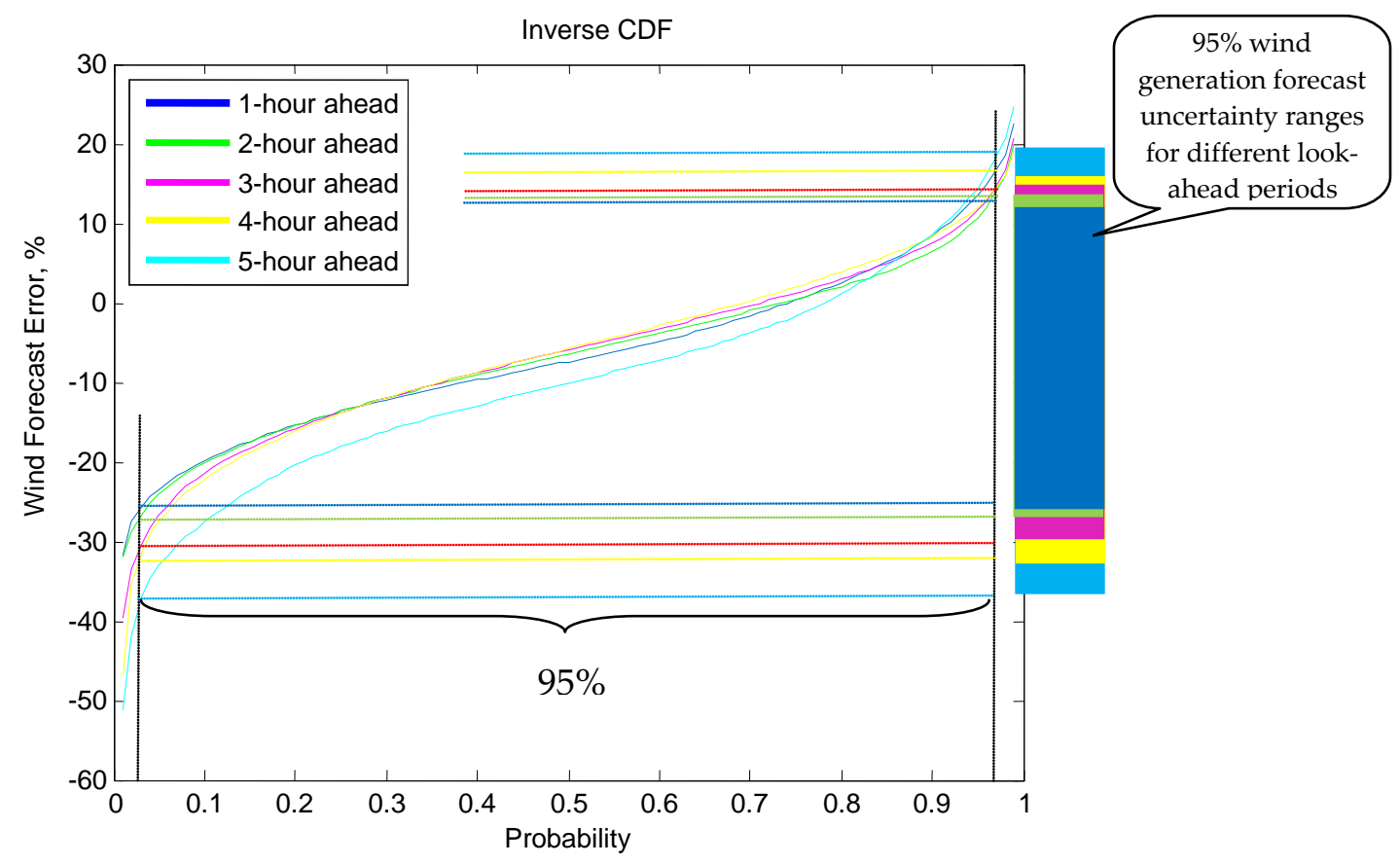

\section{Assessment of Ramping Uncertainties}

Assessment of ramping requirements is very important in case of integration of large amounts of wind generation into a power system. Sudden wind generation ramps can happen frequently and cause additional need for fast responsive generation units available on-line.

The required ramping capability needed to follow the net load curve, which covers all system imbalances, can be derived from the shape of the regulation and load following curves - see details in Makarov et al. (2009). The "swinging door" algorithm is proposed for this purpose (Makarov et al. 2009).

Figure 15 demonstrates the idea of the "swinging door" approach. A point is classified as a "turning point" whenever the next point in the sequence causes any intermediate point to fall outside a parallelogram defined by the admissible accuracy range $\pm \varepsilon \Delta \mathrm{G}$. For instance, for point 3 , one can see that point 2 stays inside the window $a b c d$. For point 4 , both points 2 and 3 stay within the window abef. But for point 5 , point 4 lies outside the window defined between points 1 and 5, and therefore point 4 is marked as a turning point.

Based on this analysis, we conclude that points 1,2, and 3 correspond to the different magnitudes of the regulation signal, $\pi 1, \pi 2$ and $\pi 3$, whereas the ramping requirement at all of these points is the same, $\rho_{1-3}$ (see Figure 16) The swinging door algorithm also determines the ramp duration $\delta$. 
Figure 15: Concept of the "Swinging Door" Algorithm

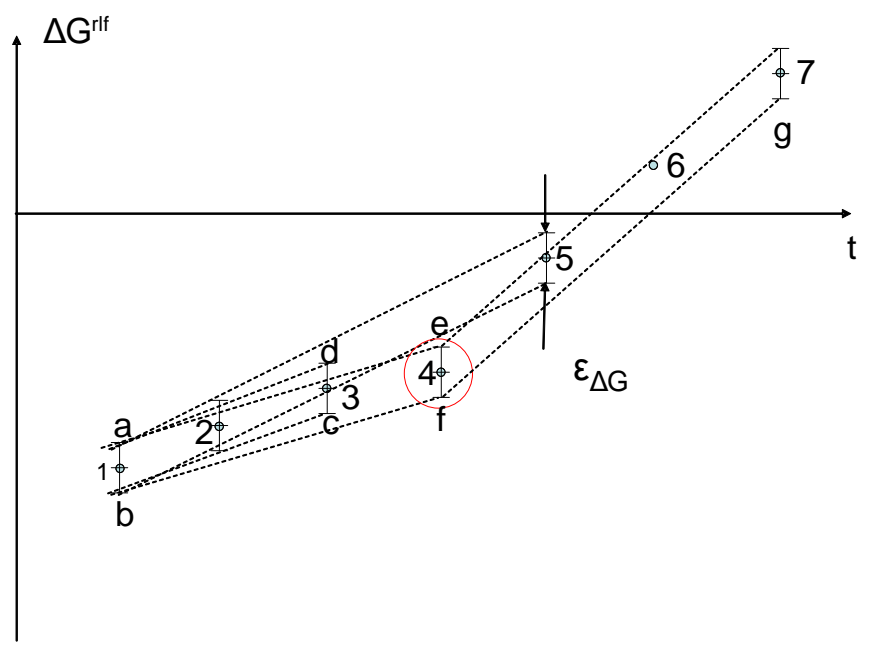

Figure 16: "Swinging Door" Algorithm - Obtaining Capacity, Ramp, and Ramp Duration

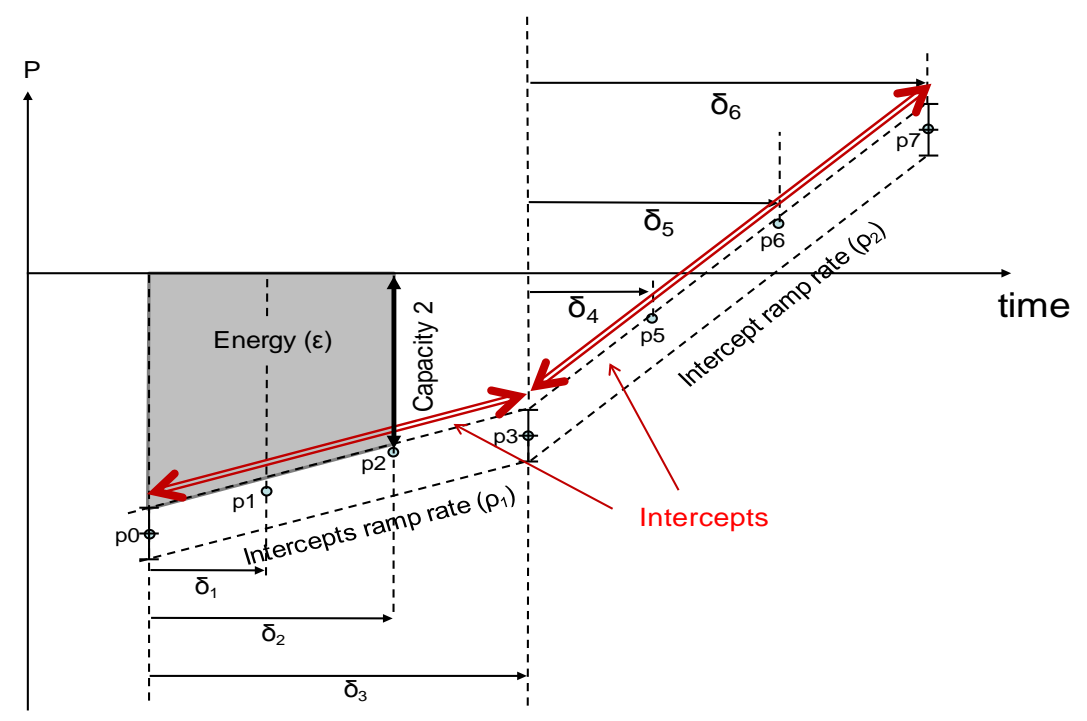

The regulation capacity and ramping requirements are inherently related. Insufficient ramping capability could cause additional capacity requirements. A multivariable statistical analysis can be applied to provide a concurrent consideration of the regulation and load following capacity, ramping, and ramp duration requirements. For the regulation/load following requirement curve, the "swinging door" algorithm is applied to determine the sequences of its magnitudes and ramps, $\pi_{1}, \pi_{2}, \ldots, \rho_{1}, \rho_{2}, \ldots$, and $\delta_{1}, \delta_{2}, \ldots$. The triads $\left(\pi_{i}, \rho_{i}, \delta_{i}\right)$ can be used to populate the three-dimensional space of these parameters (Figure 17). Let us define a rectangular box in the space that contains a certain percentage of the points. If a point lies outside the box, the regulation/load following requirements are not met at this point. We will require that this 
probability must be below a certain minimum probability, $P_{\min }$. Our task is to find a position of the walls of the probability box that corresponds to a given $P$ min. For given ranges of these three parameters, $\Delta \pi, \Delta \rho$ and $\Delta \delta$, a box can be plotted in this space, so that some triads are inside the box $\left(N_{\text {in }}\right)$, and some are outside $\left(N_{\text {out }}\right)$. This approach helps determine the probability of being outside the box,

$$
p_{\text {out }}=\frac{N_{\text {out }}}{N_{\text {out }}+N_{\text {in }}}
$$

For example, assume that the confidence level for the analysis is established at $94 \%$. Then for each dimension of the box, we can assign equal probability for finding a point outside the box due to any of the three possible reasons: insufficient generation capacity (incremental or decremental), insufficient ramping capability (upward and downward), or insufficient ramp duration capability. This results in a requirement that only $1 \%$ of the points should be left outside the two sides of the box along any of the analyzed coordinates while adjusting its walls. Of course, we should be careful not to double or triple count points that are found outside of the box due to more than one reason. The resulting size of the box determines the ranges of the generation requirements for the capacity, ramp, and ramp duration characteristics that are sufficient to meet the system needs in $94 \%$ of the cases.

Figure 17: Concurrent Consideration of the Capacity, Ramping and Duration Requirements

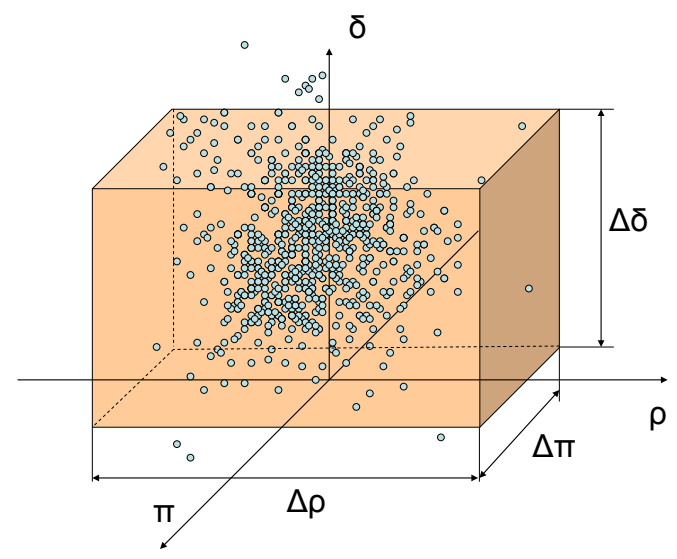

\section{"Flying Brick" Method}

Figure 18 illustrates the idea of the flying brick method (Makarov et al. 2011). Three uncertainty ranges, i.e., capacity, ramp rate and ramp duration requirements, are represented as a threedimensional probability box, i.e. the flying brick. The blue curve shows the generation requirements that meet the expected net load. The red curve refers to actual net load, which can deviate from its expected values. Suppose $t_{0}$ is the current moment. At this point, the multivariable statistical analysis is applied to forecast errors for different look-ahead intervals. 
The worst combinations of the three requirements shown by vertices of the probability box provide generation characteristics needed to meet system requirements with a certain level of confidence. For each subsequent time interval, the probability box is built based on threedimensional CDFs.

Figure 18. Concept of the "Flying Brick" Method

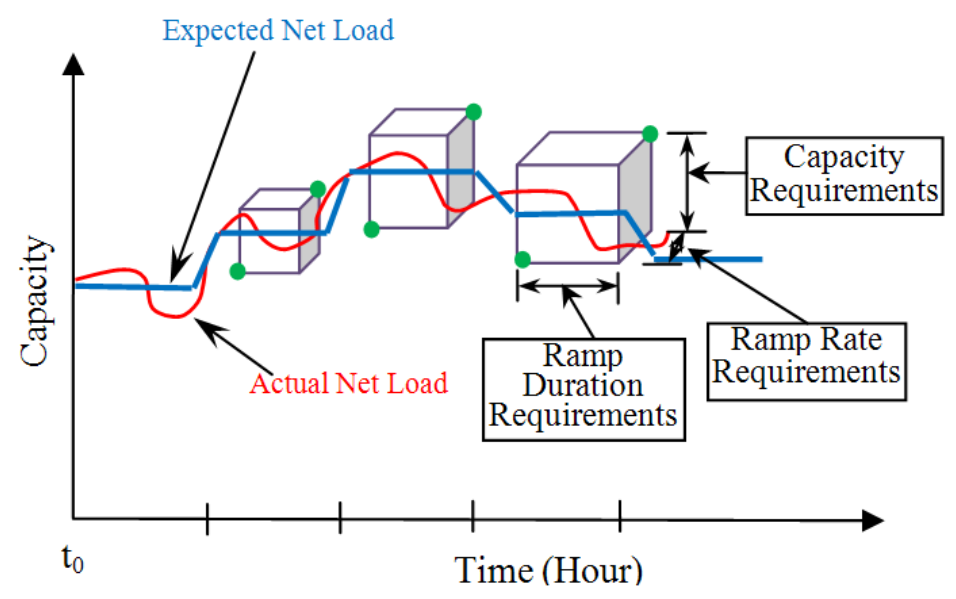

Figure 19 presents the ramping requirement PDFs for different ramp durations. Inverse CDF functions of the ramp-rate distribution for different ramp durations, obtained using the "flying brick" approach, are presented in Figure 20. Evaluation of uncertainty ranges for ramping requirements is similar to evaluating capacity requirement. Ramping requirement uncertainty ranges evaluated at the 95\% confidence level are shown in Figure 20. It can be observed that the ramping ranges depend on ramp durations, and ramping requirements become lower for longer ramp durations. 
Figure 19: Ramping Requirement PDFs for Different Ramp Durations

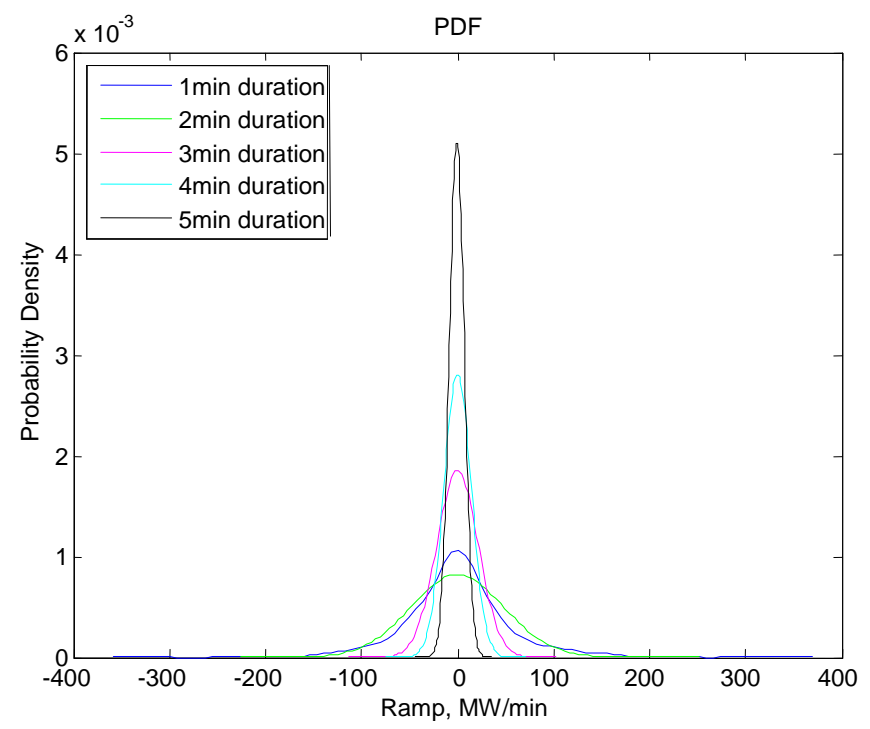

Figure 20: Ramping Requirement Inverse CDFs for Different Ramp Durations and 95\% Confidence Intervals

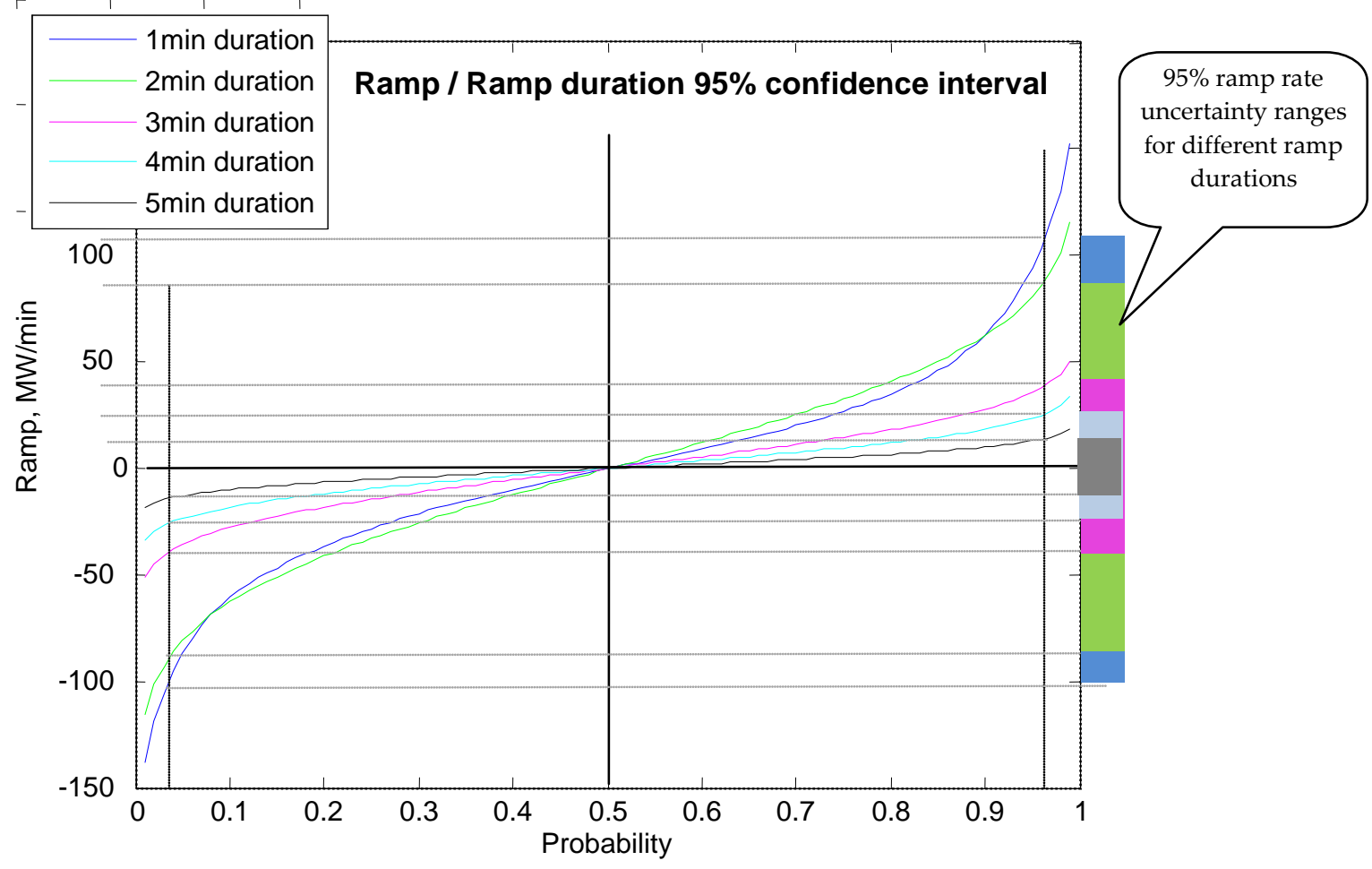

\section{Evaluation of Generation Requirements}

Evaluation of generation requirements includes an assessment of generation capacity requirements and generation ramping requirements. Evaluation of balancing capacity 
requirements is shown in Figure 21. The blue line corresponds to the generation schedule. The hour-ahead schedule with 1-hour resolution is considered in this example. Uncertainty ranges are calculated for each scheduling (dispatch) interval using individual statistical characteristics for a specified look-ahead horizon and taking into account the level of predicted wind generation, as it is obtained by the statistical analysis of retrospective information.

The following information is used:

- Load

O Actual load

o $1(2,3,4,5)$-hour-ahead load forecast

- Wind generation

0 Actual wind generation

o $1(2,3,4,5)$-hour-ahead wind generation forecast

- Solar generation

0 Actual wind generation

o 1(2,3,4,5)-hour-ahead wind generation forecast

- Interchange schedule

- Generation schedule.

Building the resulting uncertainty characteristics is a repetitive process. The generation schedule, load and wind generation forecasts, and statistical characteristics of the retrospective data are continuously updated. A sliding window with a user-specified refreshment rate is used to acquire continuously updated statistical information. The uncertainty ranges are also updated, taking into account changing generation schedules, load forecast, and wind generation forecast and their statistical characteristics. 
Figure 21: Evaluation of Capacity Requirements

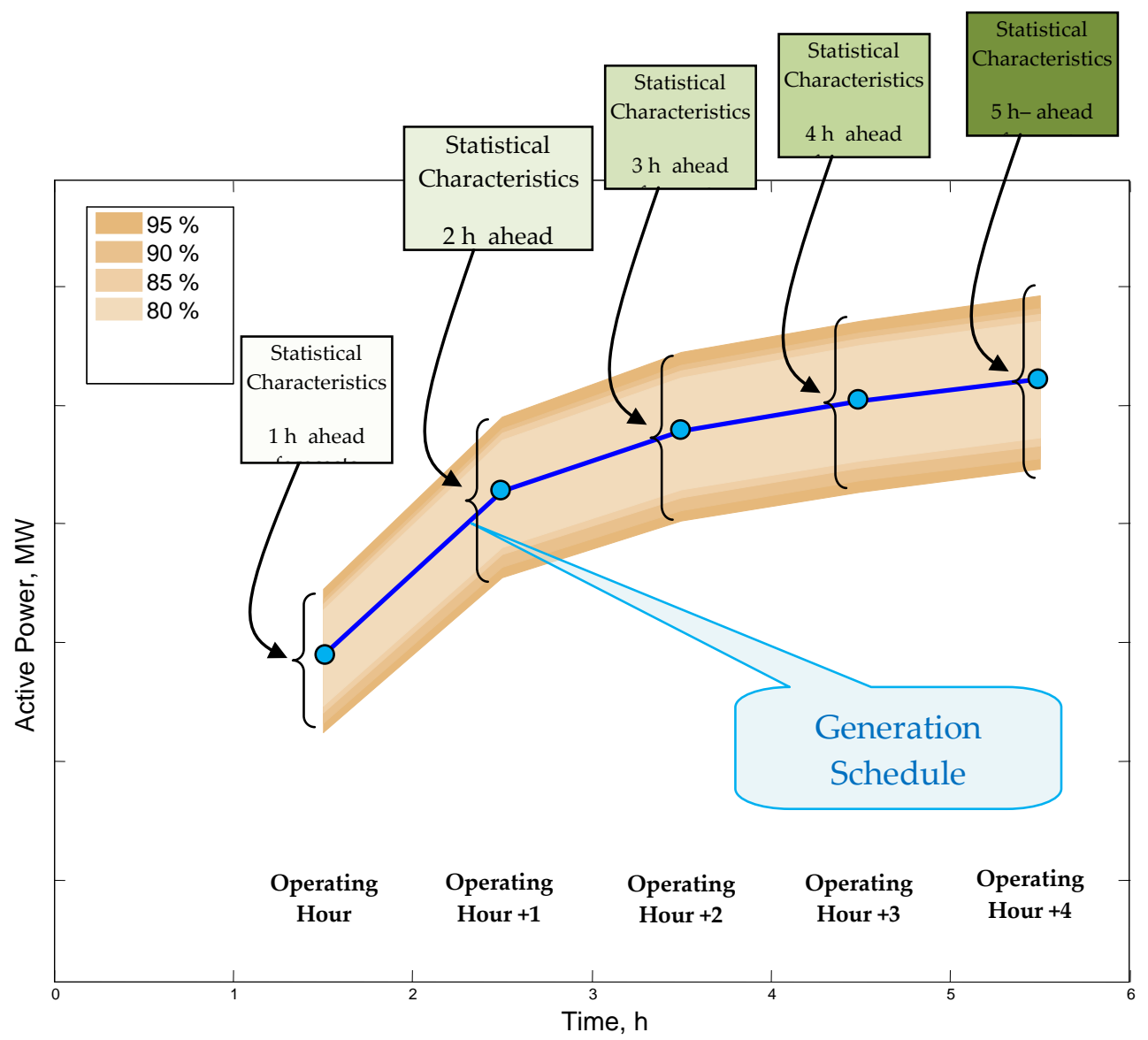

\section{Uncertainty Range Validation Approach}

To validate the accuracy of the uncertainty model of generation requirements, a validation approach has been developed.. It is based on comparing the predicted uncertainty ranges against the actually observed ranges for the same dispatch intervals. The algorithm includes the following steps.

1. Acquire retrospective statistical information using the sliding window technique. The sliding window is updated hourly (or according to some other specified refreshment rate).

2. Perform a statistical analysis of the data acquired in Step 1. The derived statistical characteristics are also updated hourly (or according to a user-specified refreshment rate).

3. Evaluate uncertainty intervals for the future generation requirements using the statistical characteristics obtained in Step 2. Uncertainty intervals are also updated according to a specified refreshment rate.

4. When the predicted dispatch interval is reached, overlay the actual generation values over the previously forecasted uncertainty intervals, as shown in Figure 22, and determine which predicted uncertainty interval the actual generation value belongs to. Put this information 
into the validation table (Table 1). Note that for different look-ahead forecasted intervals, different tables should be used.

Figure 22: Validation Procedure

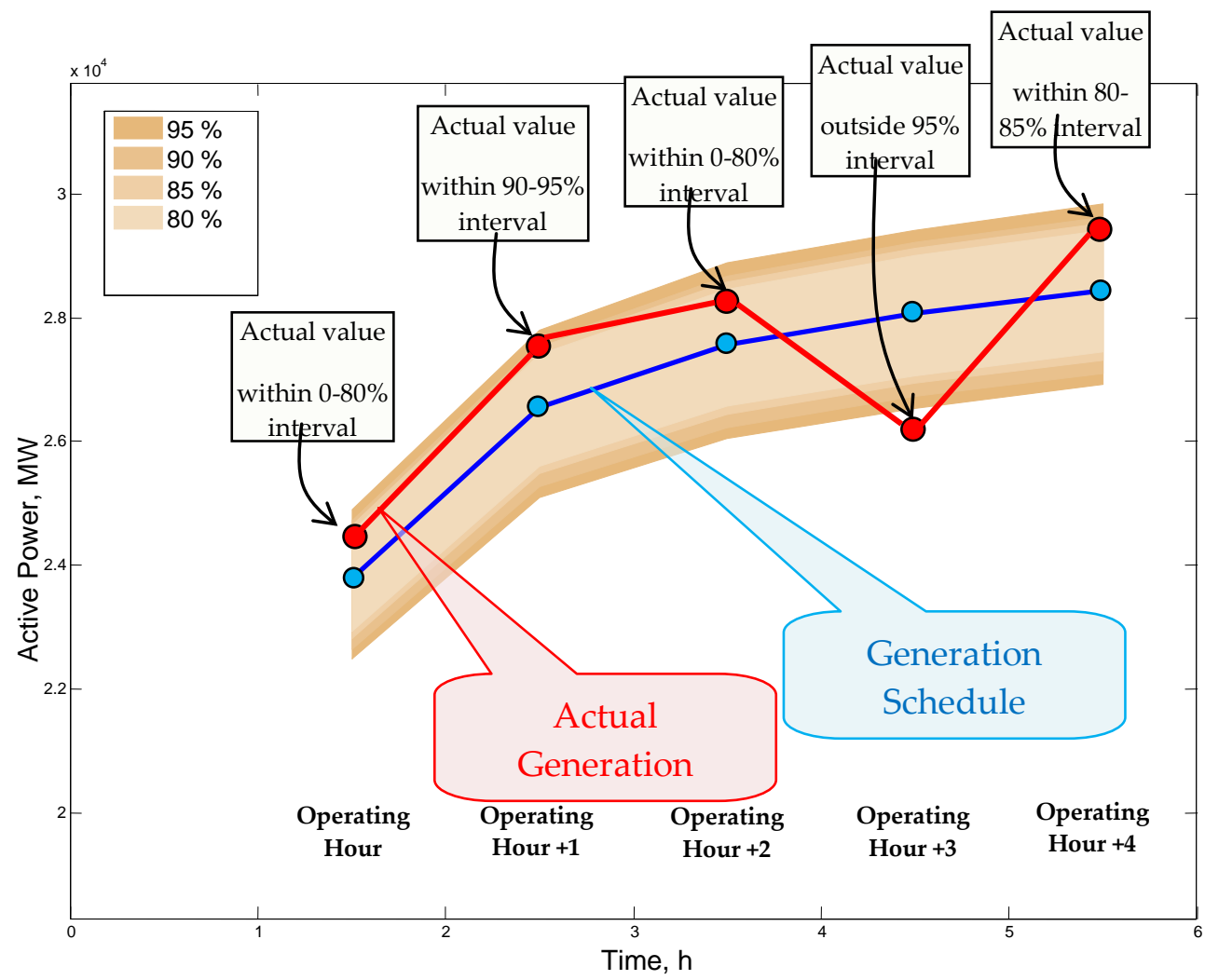

At the end of simulation, the following calculations are made:

1. Count how many points belong to a predicted interval with a specified confidence level, and calculate the percentage of points found within the interval (Table 1).

2. Compare the obtained percentages with targeted percentage values. The targeted percentages correspond to the confidence level of the interval. For example, for the 0 to $80 \%$ confidence interval, the targeted value is equal to $80 \%$, and for 80 to $85 \%$ uncertainty interval, the targeted value is equal to $5 \%$, etc. (see Table 1 ).

The uncertainty algorithm is validated if the calculated percentages and the targeted percentages are close. 
Table 1: Example of Validation Table (1-Hour Ahead Forecast)

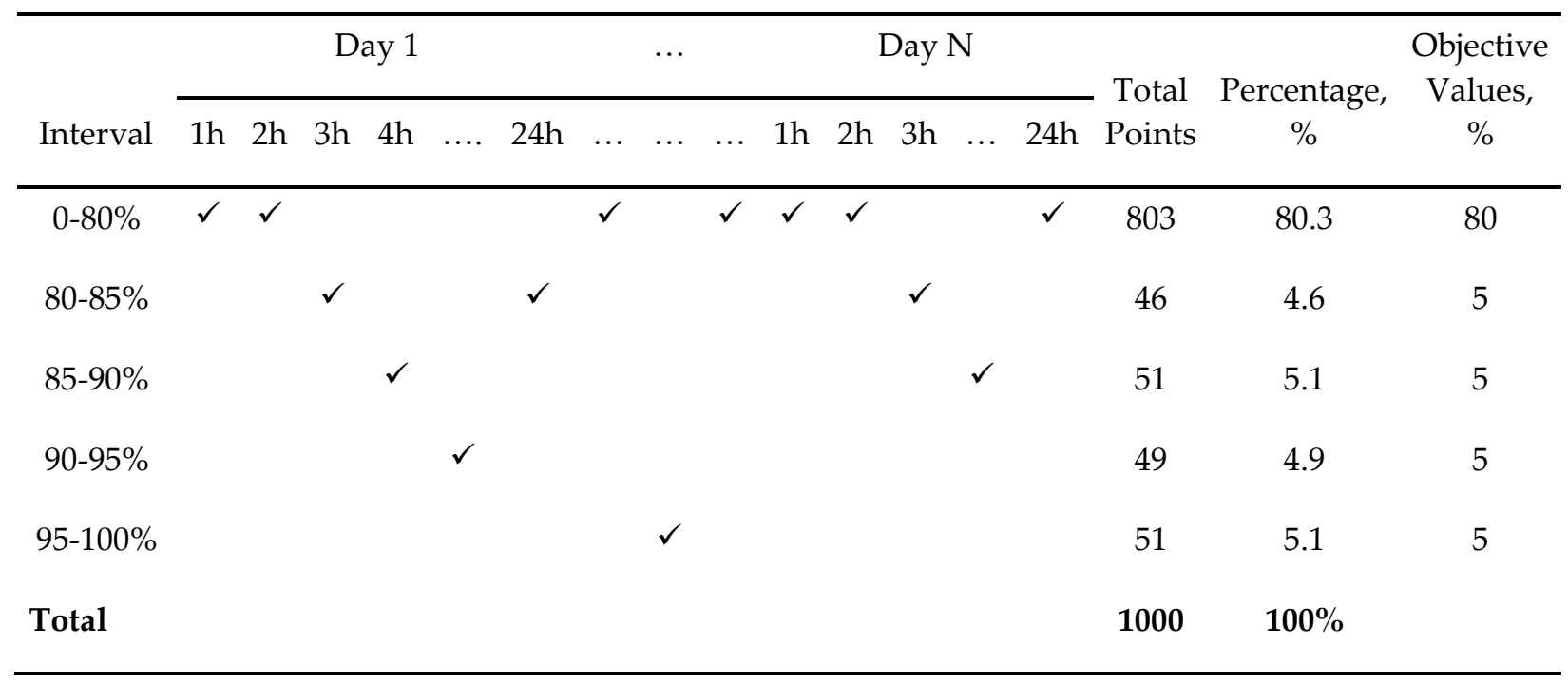

\section{Framework of Probabilistic Tool Integration}

Figure 23 shows the concepts of the three levels of integration, namely passive integration, active integration, and proactive integration.

1. Passive Integration (Level I)

Passive integration is the initial step and the simplest way of integration to bring awareness of wind and load forecast uncertainties into a control center through visualization and alarming. In passive integration, displays with look-ahead capacity and ramping requirements are provided to the real-time operators for better situational awareness. They help operators assess balancing needs and take preventive actions to mitigate potential balancing energy deficiencies. 
Figure 23: Flowchart of EMS Integration for the Uncertainty Model

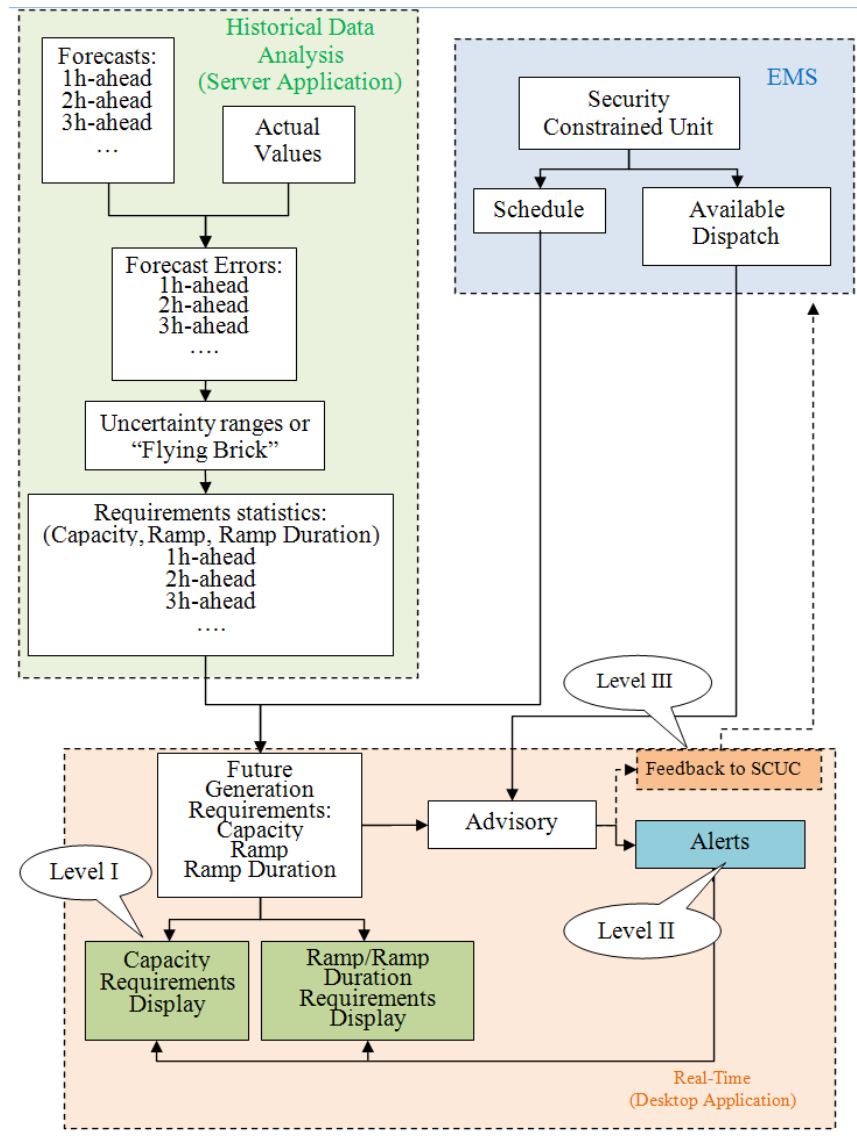

2. Active Integration (Level II)

The active integration is more comprehensive than passive integration. The active integration uses uncertainty information to re-run existing grid operation functions such as UC and economic dispatch (ED) processes for the worst-case combination of uncertainties within the specified confidence level. The tool displays warning messages about potential threats to the power system if the UC or ED procedures cannot find solutions for the worst cases. It also provides operators with advisory information regarding the actions that could be taken to avoid potential problems. The active integration does not modify the UC and ED procedures. Instead, it uses existing processes in the EMS system to check the sufficiency of balancing resources within the range of uncertain system requirements.

3. Proactive Integration (Level III)

Proactive integration is the most comprehensive level of EMS integration, because it not only interacts with UC, ED, and other applications in the EMS system, but also modifies the algorithms. New constraints based on uncertainty range evaluations are incorporated into the $\mathrm{UC}$ and ED processes. For example, the uncertainty ranges of the capacity and ramping requirements can be incorporated in the UC process as a part of reserve requirements as shown in Figure 24. 
Figure 24: Flowchart of Proactive Integration

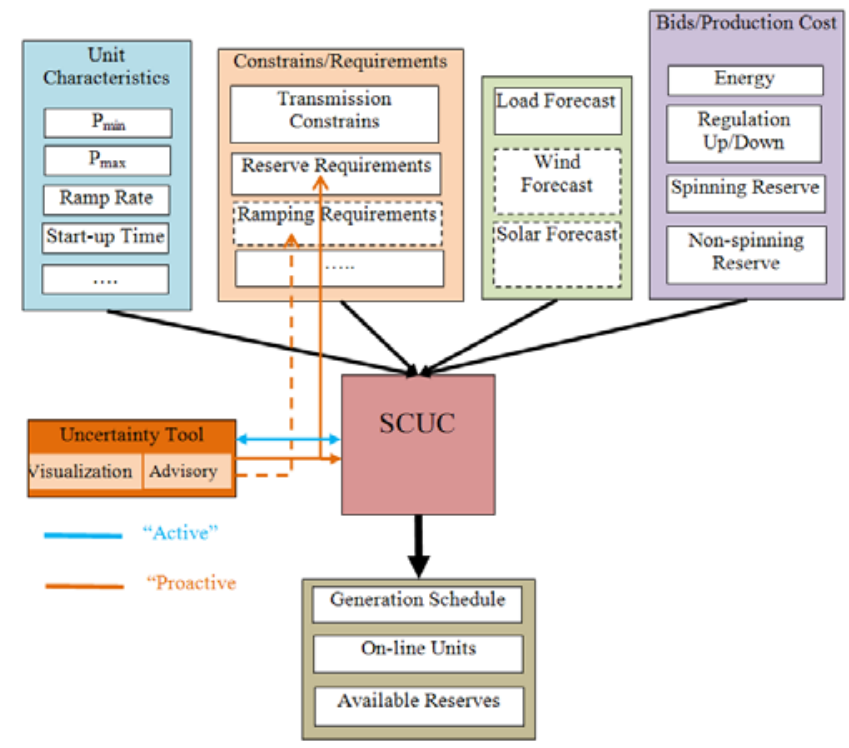

\section{Real-Time Forecast Errors}

\section{Wind and Solar Generation Forecast Errors}

Currently, the real-time wind and solar generation forecasts are not provided or included in the CAISO real-time dispatch process. Instead, the simple persistence model is implicitly used (Figure 25). Practically this means that, for example, for a 5-minute dispatch interval $[t+5$, $t+10]$, the implicit real-time wind (or solar) generation forecast is assumed to be equal to the average actual wind (or solar) generation in the interval $[t-5, t]$.

Figure 25. Simple Persistence Model Illustration

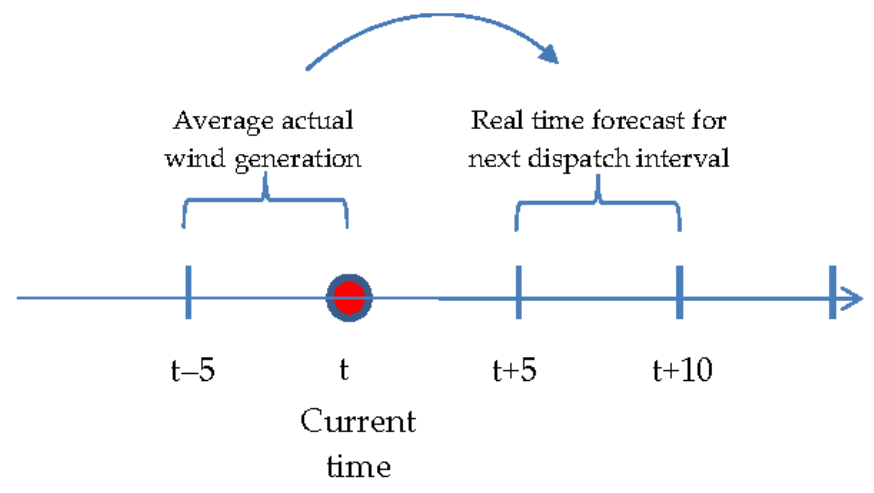

Figure 26 and Figure 27 show CAISO's real-time wind and solar generation forecast errors calculated using the persistence model. 
Figure 26: Real-Time Wind Generation Forecast

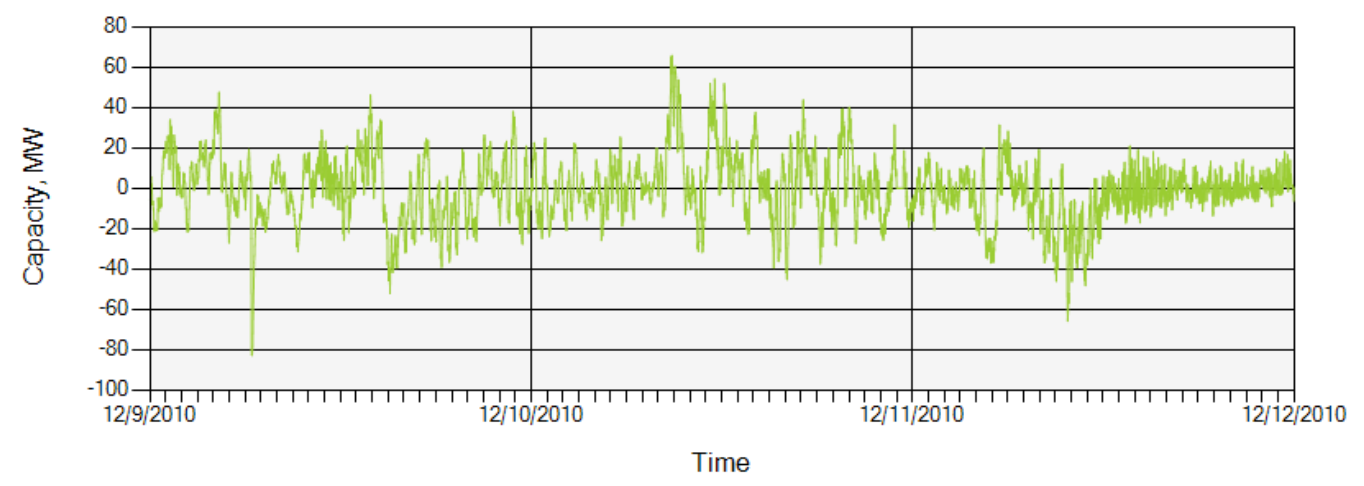

— WindForecastError

Figure 27: Real-Time Solar Generation Forecast

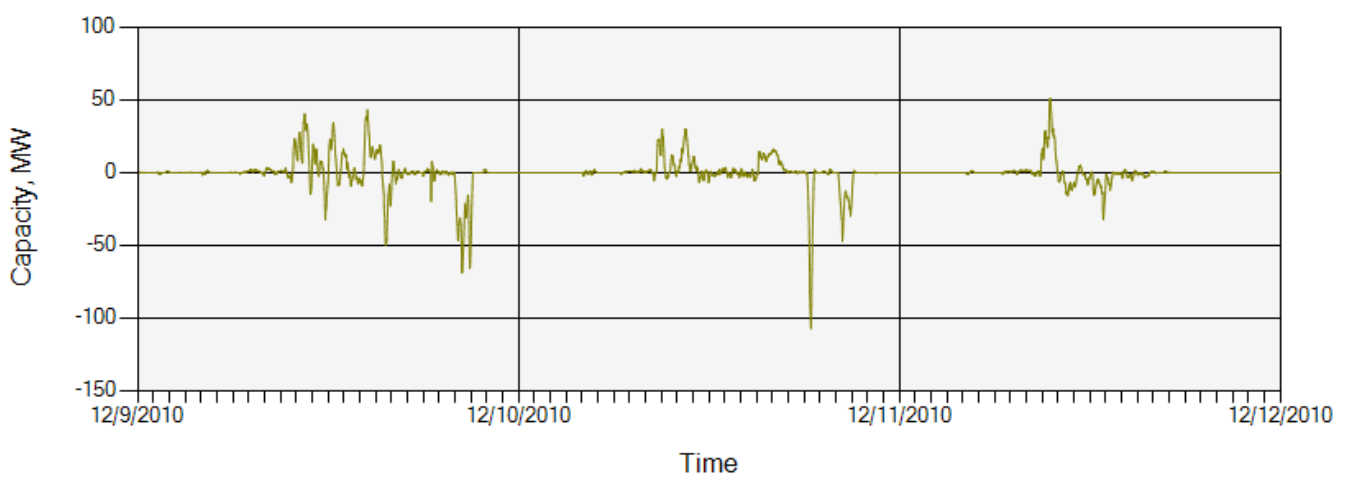

- SolarForecastError

\section{Load Forecast Errors}

CAISO uses an adaptive load forecasting system (ALFS) to predict load in real time. The forecast is updated every 5 minutes up to $65 \mathrm{~min}$ ahead. Figure 28 presents the observed CAISO real-time load forecast errors for 12/9/2010 - 12/12/2010.

Figure 28. Real-Time Load Forecast Error

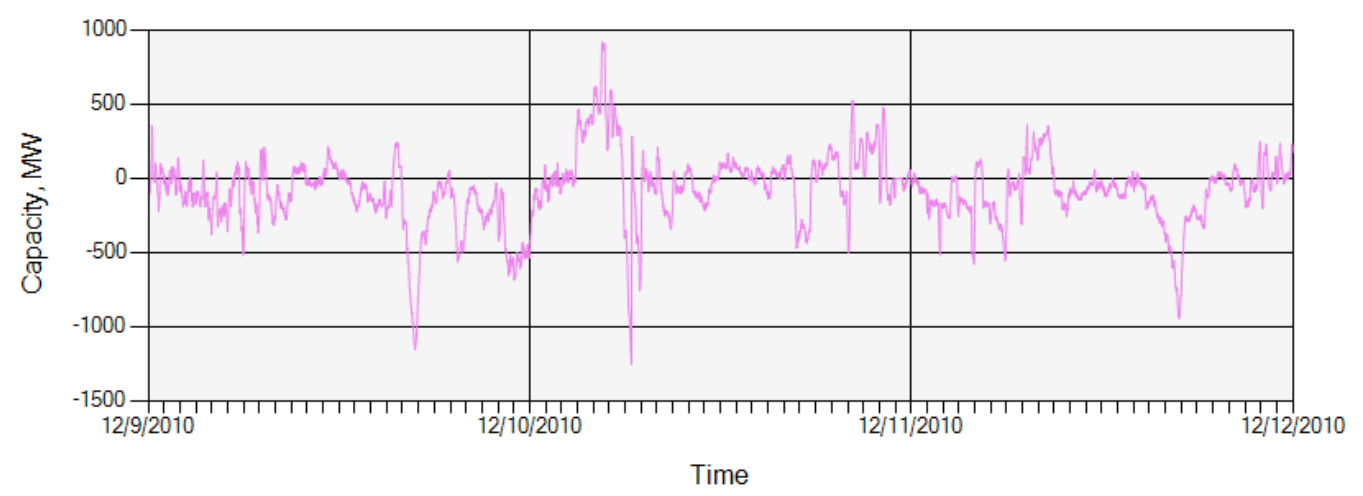

- LoadForecastError 


\section{CHAPTER 3: Review of Current Operating Practices at CAISO}

To incorporate the proposed probabilistic tool into the real EMS systems, we need to know the actual procedures for generation scheduling and dispatch used by the balancing authorities where this integration will take place. In this section, the operating practices at the CAISO are briefly reviewed.

CAISO runs different generation schedules in the day-ahead market and real-time market to make sure that the energy and reserve requirements including regulation up, regulation down, and ramping requirements are ultimately met in real-time operation.

Figure 29 shows the CAISO market timeline. The CAISO scheduling process includes dayahead market (DAM), real-time unit commitment (RTUC), short-term unit commitment (STUC), and real-time economic dispatch (RTED). Although regulation (REG) capacity is procured in the day-ahead market, it is controlled by the EMS AGC system, rather than the market software (CAISO 2006).

Figure 29: An illustration of CAISO Operating Timelines (not to scale)

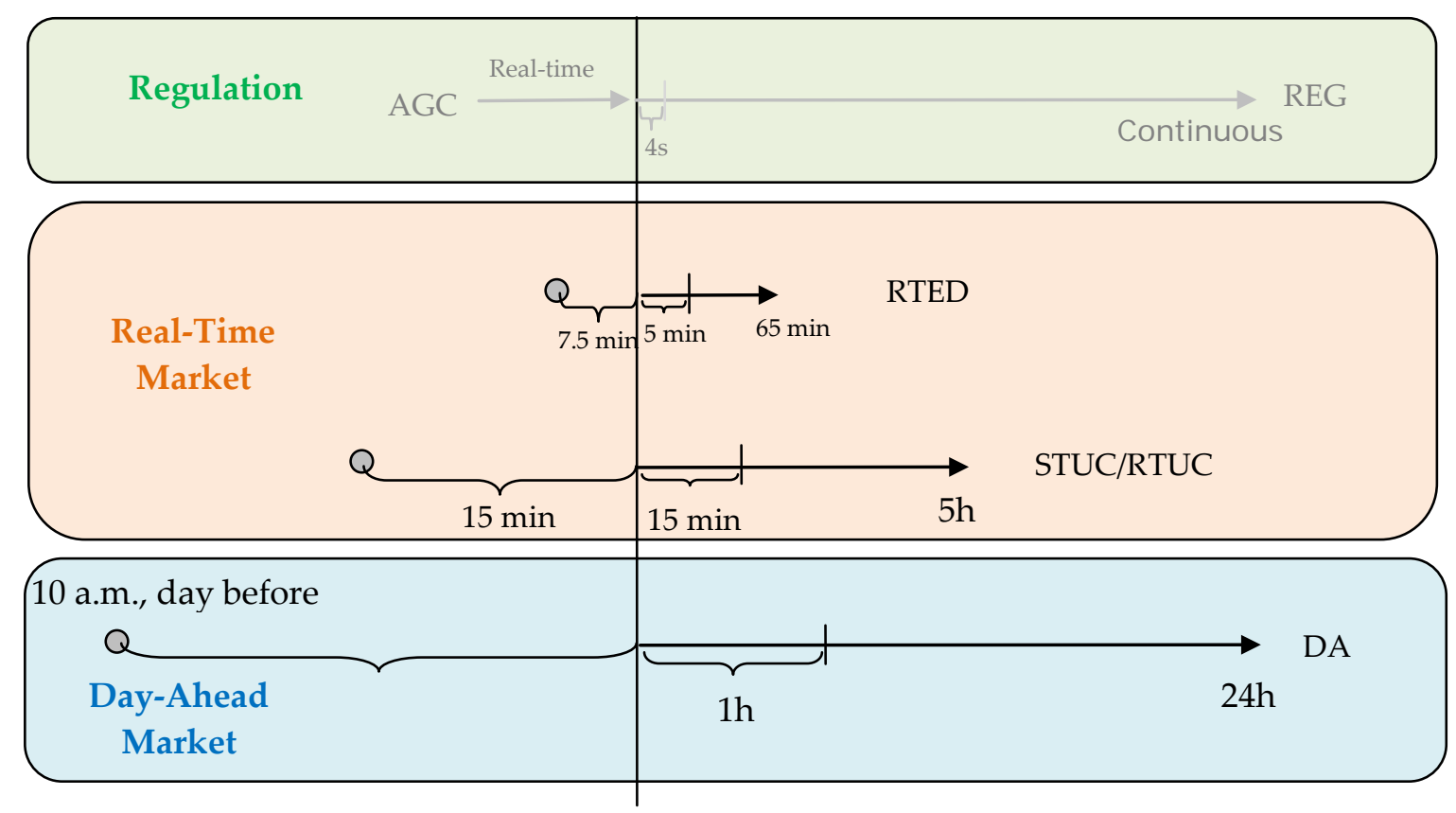

The regulation capacity is procured day-ahead for each operating hour of the next operating day. The additional ancillary services (AS) also can be procured in the real-time market (RTM) to meet additional AS requirements. The AS include: regulation-up reserve, regulation-down 
reserve, spinning reserve and non-spinning reserve. Time characteristics of the scheduling and dispatch processes are given in Table 2. A detailed picture of the CAISO market timeline is presented in Figure 30.

Table 2: Time Characteristics of the Scheduling Process at CAISO

\begin{tabular}{|c|c|c|c|c|c|}
\hline Element & Acronym & Start time & Interval & Frequency & Time horizon \\
\hline $\begin{array}{c}\text { Day-Ahead } \\
\text { Market }\end{array}$ & DAM & $\begin{array}{c}\text { Closes at 10:00 } \\
\text { on the day } \\
\text { before }\end{array}$ & $1 \mathrm{~h}$ & Every day & $24 \mathrm{~h}$ \\
\hline $\begin{array}{l}\text { Short-Term } \\
\text { Unit } \\
\text { commitment }\end{array}$ & STUC & $\sim 75$ min before & $15 \mathrm{~min}$ & hourly & up to 5 hours \\
\hline $\begin{array}{l}\text { Real-Time Unit } \\
\text { commitment }\end{array}$ & RTUC & - & $15 \mathrm{~min}$ & $15 \mathrm{~min}$ & $\begin{array}{l}4-7 \times 15 \mathrm{~min} \\
\text { interval }\end{array}$ \\
\hline $\begin{array}{l}\text { Real-Time } \\
\text { Economic } \\
\text { Dispatch }\end{array}$ & RTED & $7.5 \mathrm{~min}$ before & $5 \mathrm{~min}$ & Every $5 \mathrm{~min}$ & $65 \mathrm{~min}$ \\
\hline Regulation & REG & - & $4 \mathrm{~s}$ & $4 \mathrm{~s}$ & - \\
\hline
\end{tabular}

The CAISO RTM consists of several applications, three of which, including STUC, RTUC, and RTED, work together. The STUC and RTUC applications ensure there is enough on-line capacity to meet a 5-minute demand (Figure 31). The STUC is performed in the RTM to commit units and balance the system resources and demand while enforcing transmission constraints. STUC is run once an hour and looks out 5 hours to commit resources that have start-up times greater than 90 minutes.

The RTUC application runs every 15 minutes and looks out between four and seven 15-minute intervals to determine whether short-start and fast-start units need to be committed or decommitted.

The RTED process runs every 5 minutes to meet the imbalance energy requirements of the CAISO (Figure 32). This process looks ahead 65 minutes to ensure that enough capacity is on line to meet real-time demand. It is expected that wind variability and the lack of accurate wind forecasts could create challenges for the RTED application. RTED is the lowest granularity of dispatch in the ISO market except for regulating reserves, which is procured in the RTM, but is dispatched through the EMS AGC system every 4 seconds. 
Figure 30: A detailed illustration of CAISO Operating Timelines

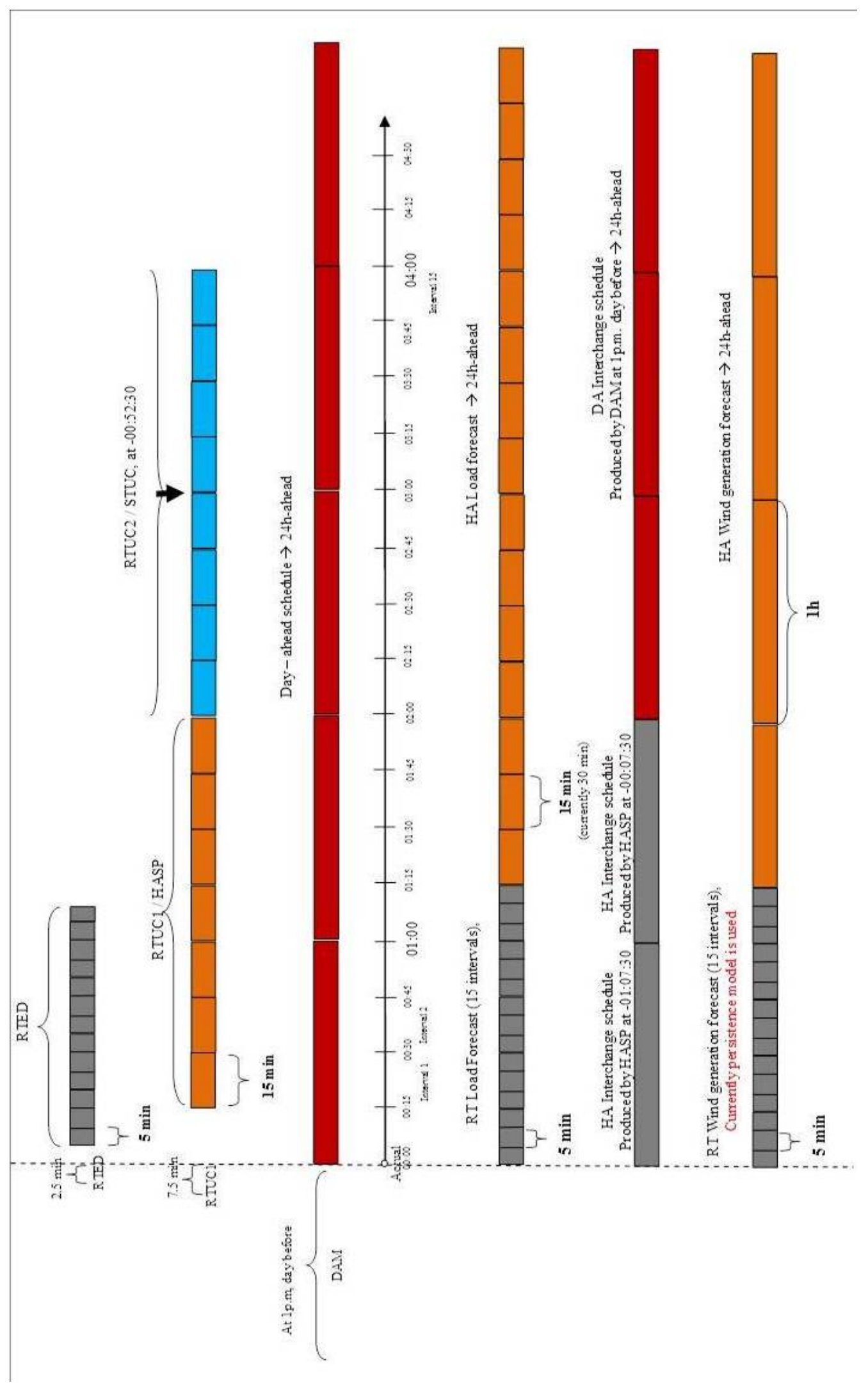


Figure 31: RTUC/STUC Timeline

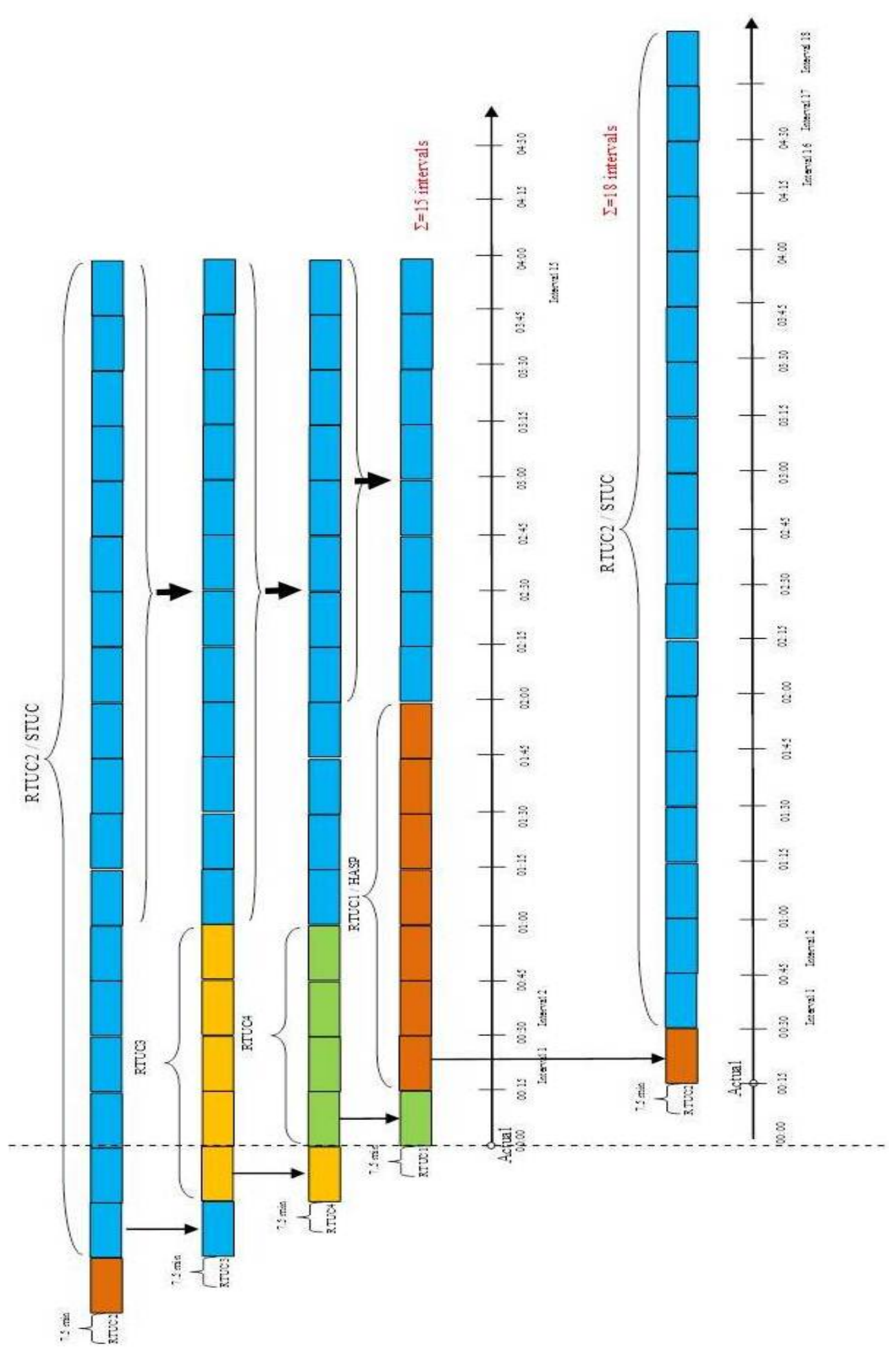


Figure 32: RTED Timeline

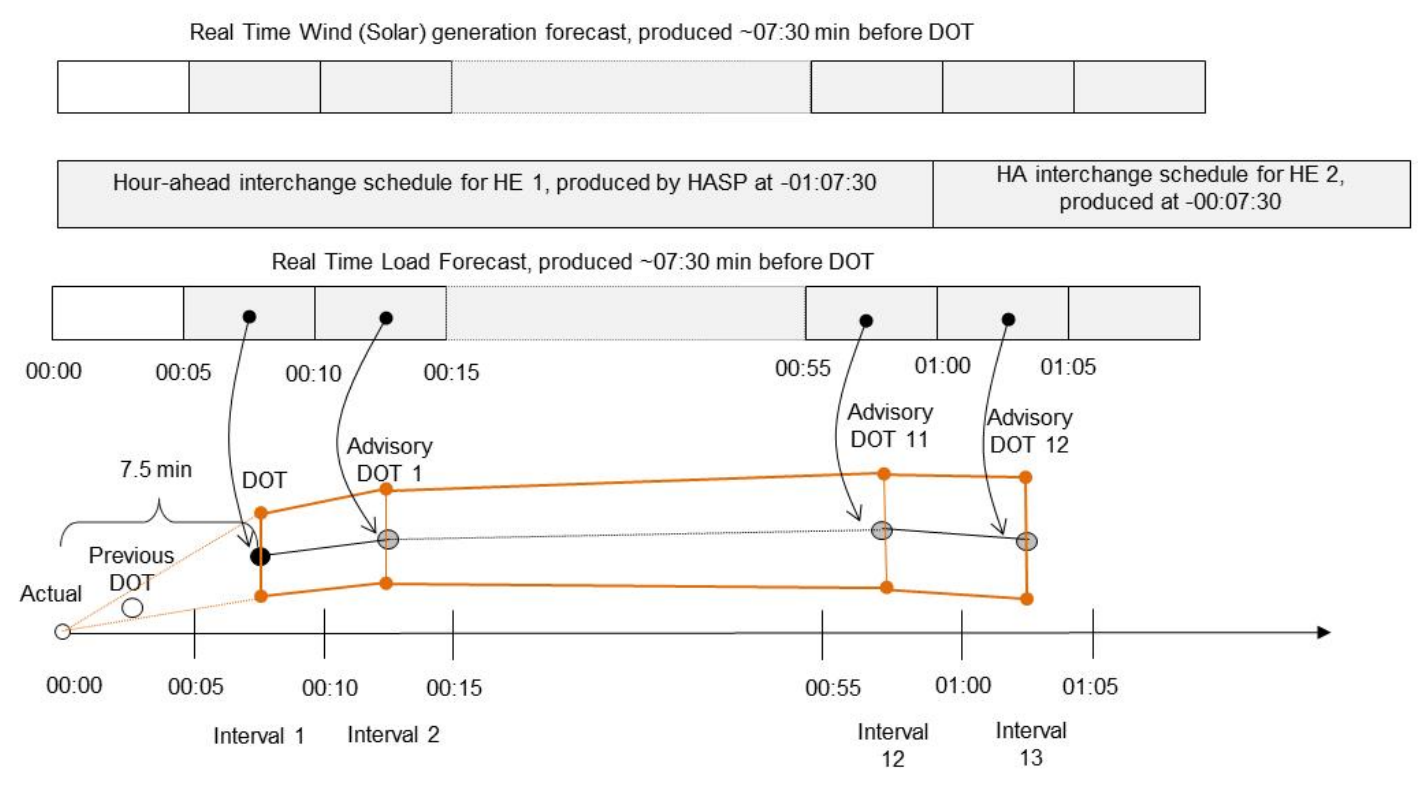

Figure 33 represents the CAISO market design generation schedules. In the day-ahead (DA) timeframe, wind and solar resources are not required to bid directly into the CAISO markets. This fact can significantly impact the unit commitment process in the DA timeframe because the CAISO must forecast the expected hourly production in the DA to ensure that enough resources are committed for the next day's operation. Similarly, the CAISO load forecast is done in the DA and real time (RT) timeframes. In the DAM, the forecast of the CAISO's hourly demand is done for three days in advance. The DA schedule is an hourly block energy schedule that includes 20-minute ramps between hours. It is provided at 10:00 a.m. the day prior to the operating day. The real-time schedule is based on STUC/RTUC timelines. RTED is provided 7.5 minutes before the dispatch operating target (DOT) and is based on real-time forecasts (Figure 32). Symmetrical ramping is used, which means that by dispatching for the average, the DOT ends in the center of the interval. In the RTM, the CAISO ALFS provides a load forecast for each 15-minute and 5-minute interval. Load and wind forecasting errors can cause the RTM application to dispatch incorrect amounts of imbalance energy needs. RTED results are 5minute dispatch instructions and advisory notices for the look-ahead timeframe.

Thus, the load-following or supplemental energy dispatches are the difference between RTED and STUC/RTUC curves. This is an instructed deviation caused by real-time dispatches. Regulation is the difference between the actual demand and the RTED curves (see Figure 33). 
Figure 33: Generation Dispatch Components

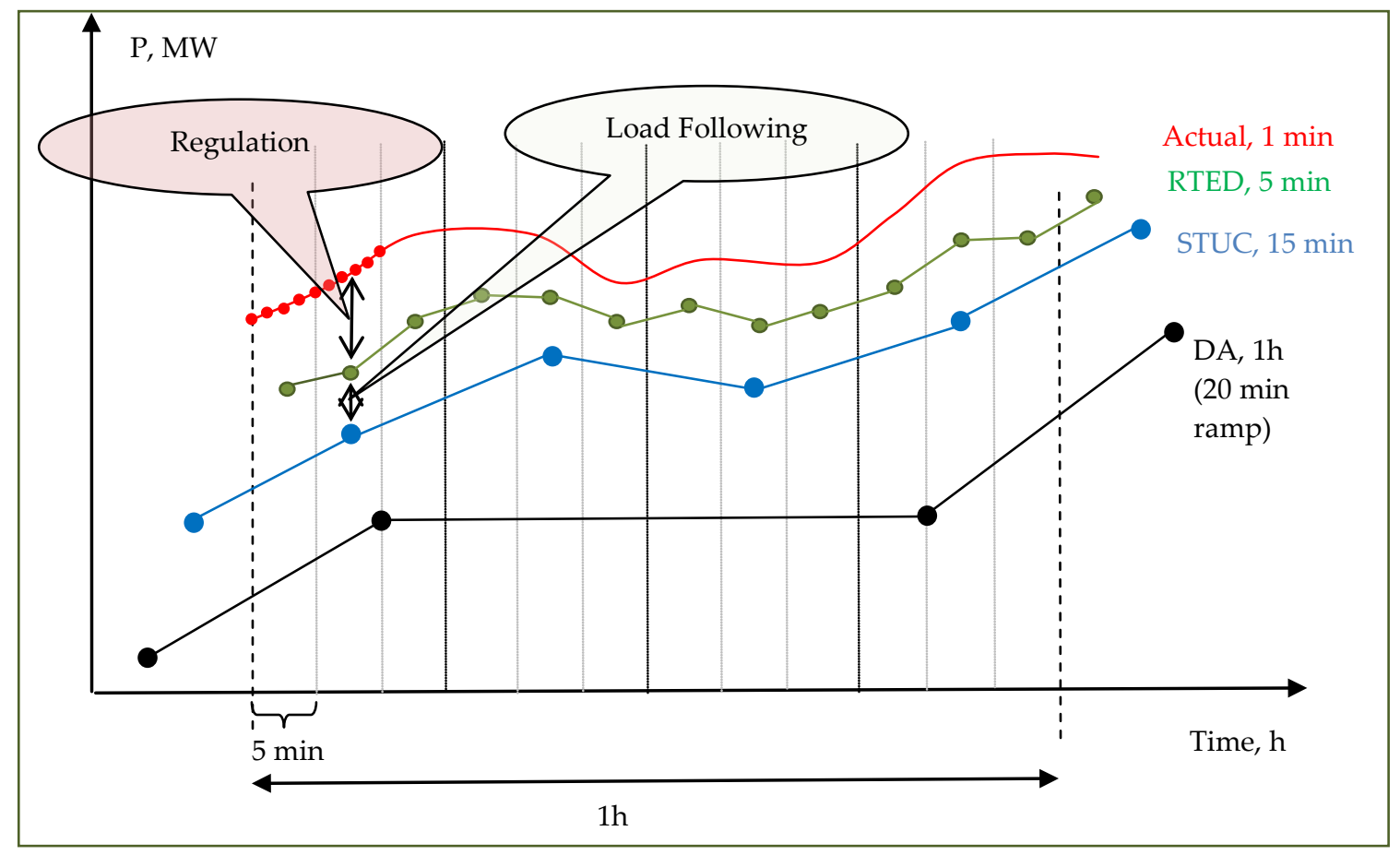




\section{CHAPTER 4: Software Tool}

A software tool for generation requirements uncertainty evaluation has been developed. The tool is based on the methodology developed in this project. The initial version was installed at the CAISO control room for testing in May 2011.

In the first phase, the plan is to operate the tool in the testing mode as a stand-alone tool. It corresponds to the passive level of integration (see Chapter 2 for details). During the testing period, the CAISO specialists will evaluate the efficiency and the usefulness of the tool. Testing will be based on analysis of retrospective data collected from different sources of information, such as CAISO's supervisory control and data acquisition/energy management system (SCADA/EMS), CAISO's market system, CAISO's master file, CAISO's wind forecast provider, etc. The prototype can help CAISO to evaluate the balancing capacity needed to mitigate negative impacts caused by unpredicted deviations of wind generation, as well as those due to inaccurate load forecast. Assuming the tool is successful in the testing phase, CAISO can decide on active or proactive integration of the tool into the CAISO's EMS system.

\section{User Interface Conceptual Design}

Information representation is an important aspect in the integration, in order to provide easy-tounderstand, real-time information to dispatchers. The design of several displays is presented in this section.

\section{Capacity Requirements Screen}

A conceptual view of the capacity requirement screen is shown in Figure 34. The capacity requirement screen contains the following information:

- generation requirements forecast (orange line)

- generation schedule to a specified time horizon (blue line)

- capacity requirements uncertainty ranges with different confidence levels associated with the generation schedule (orange ranges)

- available balancing capacity reserve in the system (gray areas)

- $\quad$ alerts and advisories.

The system would have adequate balancing reserve with a specified confidence level if the available balancing reserve (the gray area) covers the entire net load uncertainty range (the dark or light orange area). Otherwise, deficiency of balancing reserve occurs. This means that there is a certain probability that the online generation will not be able to follow the net load requirement. In this case it is necessary to commit or de-commit additional generators to achieve the desired confidence level for the balancing reserve. 
The screen is updated at a specified interval (every five minutes in real-time mode). As the screen is updated, the confidence intervals, as well as any alerts or advisories, will also be updated.

Figure 34: Capacity Requirements Display Concept

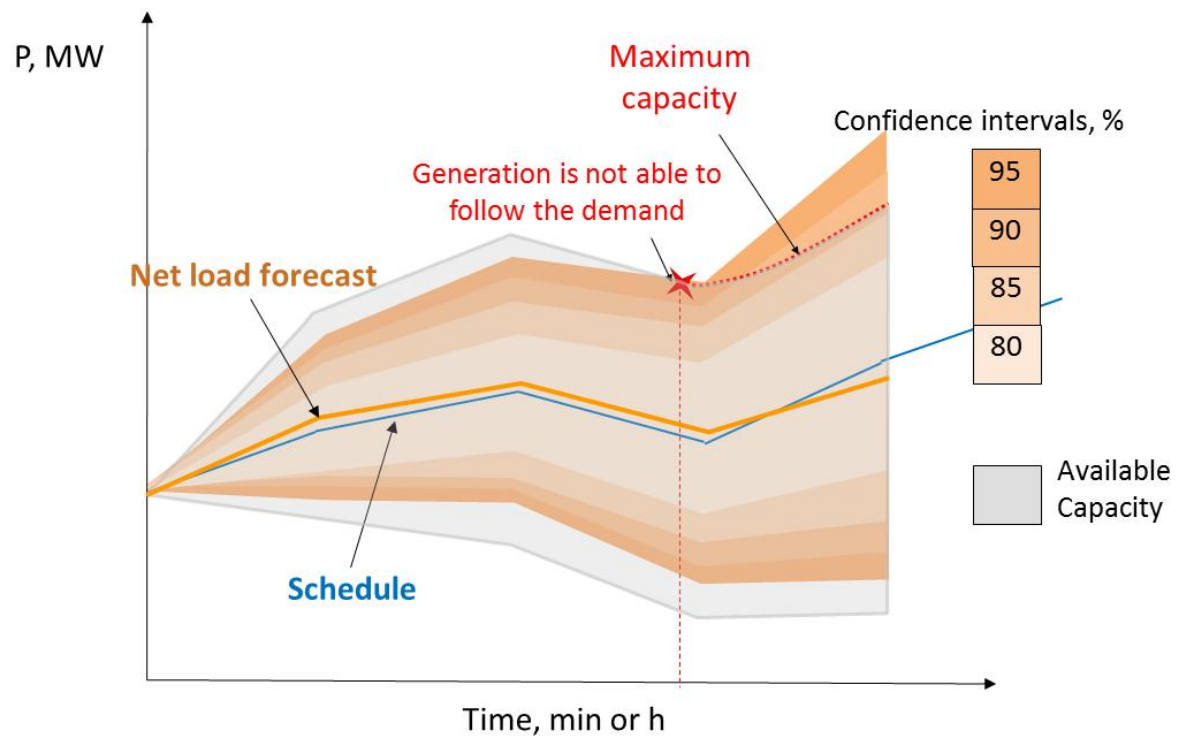

\section{Ramping Requirements Screen}

A conceptual view of the ramping requirements screen is shown in Figure 35. The ramping requirements screen contains the following information:

- Uncertainty range of ramping requirements at each operating interval for a specific time horizon (green bars).

The length of each green bar indicates the ramp rate requirements ( $\mathrm{MW} / \mathrm{min})$. The width of a bar indicates the ramp duration requirements (minutes);

- Available generation ramping capability at each operating interval for a specific time horizon (blue bars).

The length of each blue bar indicates the maximum ramp rate capability ( $\mathrm{MW} / \mathrm{min}$ ) for a given ramp duration (the width of the bar);

- Alerts and advisories (red vertical error bars).

The length of each red bar indicates the deficiency of system ramping capability in terms of ramp rate ( $\mathrm{MW} / \mathrm{min})$. 
Figure 35: Ramping Requirements Display Concept

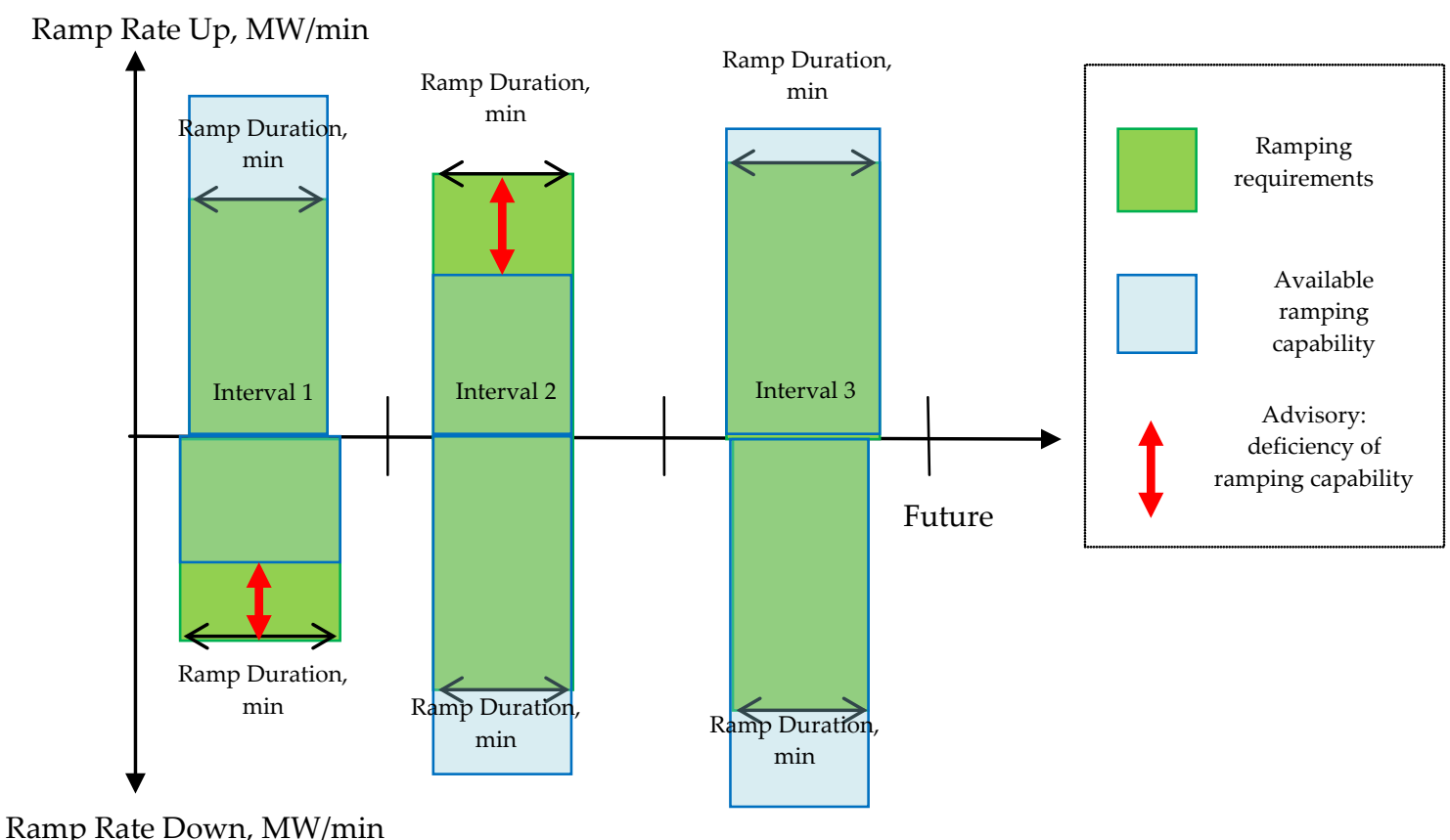

\section{Conceptual Design of Probabilistic Tool Integration}

Integration of probabilistic tools into an EMS should take into account operating practices of specific power systems. Figure 36 shows a conceptual view of the capacity requirements uncertainty evaluation based on the CAISO scheduling process. The RTED, STUC, and DAM scheduling tools (described in Chapter 3) use various forecasts, such as those that provide forecasts with different dispatch intervals for different time horizons, and those with different resolutions. Therefore, these forecasts have different accuracies, statistical characteristics, and uncertainty ranges associated with them. Figure 36 shows the uncertainty ranges as color bars for different time horizons. Different shades of each color indicate different levels of confidence. For the first 65-minute time horizon, when the scheduling is done by RTED, the uncertainty range is smaller because the forecast is more accurate compared to longer-term forecasts such as those for the 5-hour horizon and the 24-hour horizon, when the scheduling is done by STUC and DAM, respectively. RTED runs every 5 minutes, so the uncertainty needs to be evaluated at a 5-minute interval. For the 5-hour STUC and the 24-hour DAM, uncertainty can be evaluated for look-ahead intervals of 15 minutes up to hours. 
Figure 36: CAISO Capacity Requirements Uncertainty Evaluation Concept

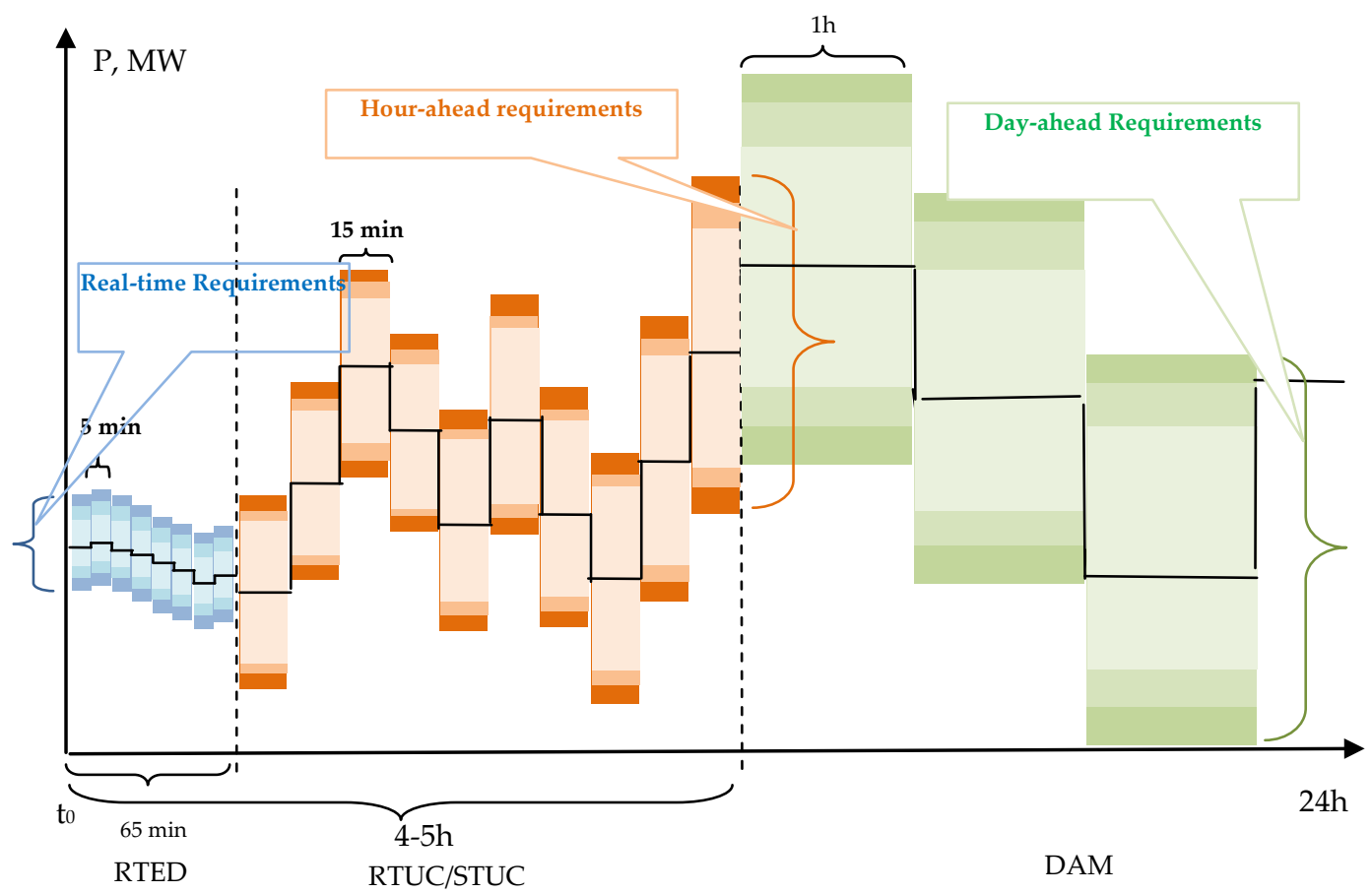

\section{Data Acquisition}

In order to model the statistical uncertainty information, large volumes of historical and realtime data are needed. As shown in Figure 37, a sliding window is used for acquiring continuous statistical information on quantities such as system load, wind and solar power generation, and generation schedules.

Data needed for the uncertainty evaluation process is stored in a database. The time-frame size and refreshment rates of sliding windows are tuned for different systems depending on their individual characteristics. This study uses a one- to two-month time frame and a $15 \mathrm{~min}$ refreshment rate for hour-ahead data and 5 min refreshment rate for real-time data.

Figure 37 represents a typical structure of the load or wind generation forecasts. The forecast resolution is the time interval between two consecutive data records. The time horizon is the length of the look-ahead interval, and the forecast update interval is the time interval between updates of the forecast. Besides the statistical information, actual measurements are also acquired. 
Figure 37: Example of Wind/Load/Solar Forecast Structure

Forecast time horizon

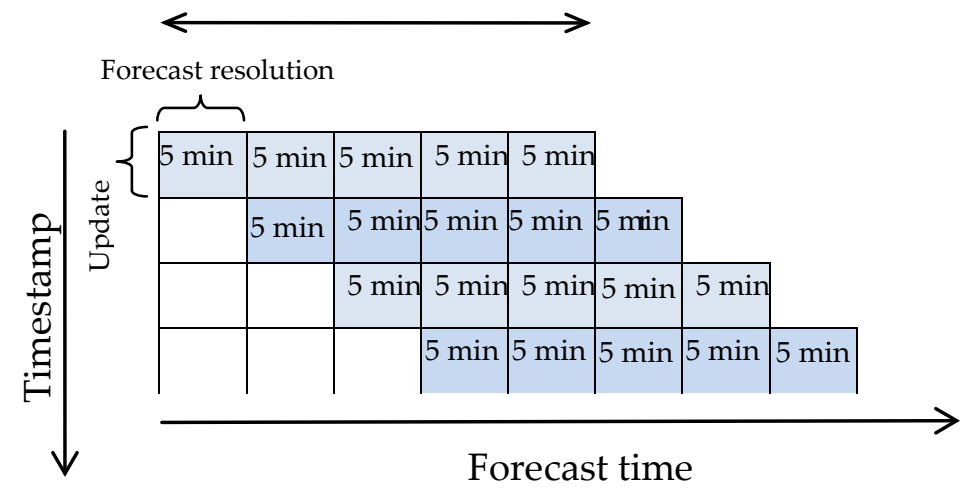

\section{Data Processing}

A visual inspection of the provided data revealed that the data sets contain instances of bad data, such as outliers, spikes and missing points. Two approaches are used to detect and eliminate bad data. The first approach identifies the bad data by detecting sudden changes. The second approach is based on a standard outlier-detection algorithm, such as the $k$-sigma criterion (sigma is the standard deviation of the examined data set).

\section{Sudden-Change Detection}

Point $x_{t}$ does not belong to time series $X$ if

Error! Objects cannot be created from editing field codes.,

where $\Delta$ max is a threshold ramp value (Figure 38).

Figure 38: Sudden-Change Detection

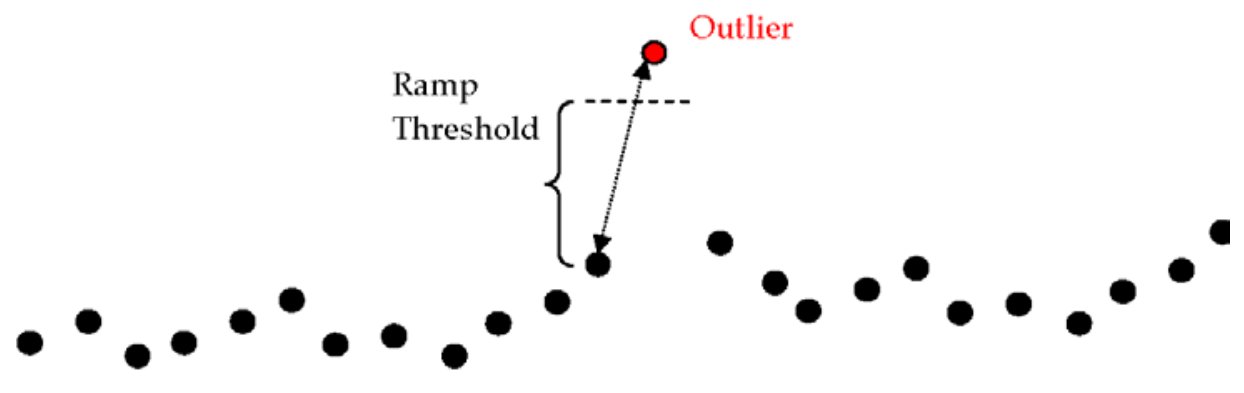




\section{Outlier Detection}

Point $x_{t}$ does not belong to time series $X$ if

$$
\left\{\begin{array}{l}
x_{t}>\mu+k \sigma \\
x_{t}<\mu-k \sigma
\end{array},\right.
$$

where

$$
\begin{aligned}
\sigma & =\text { standard deviation; } \\
\mu & =\text { mean value; } \\
k & =\text { the deviation factor (Figure 39). }
\end{aligned}
$$

Figure 39: Outlier Detection

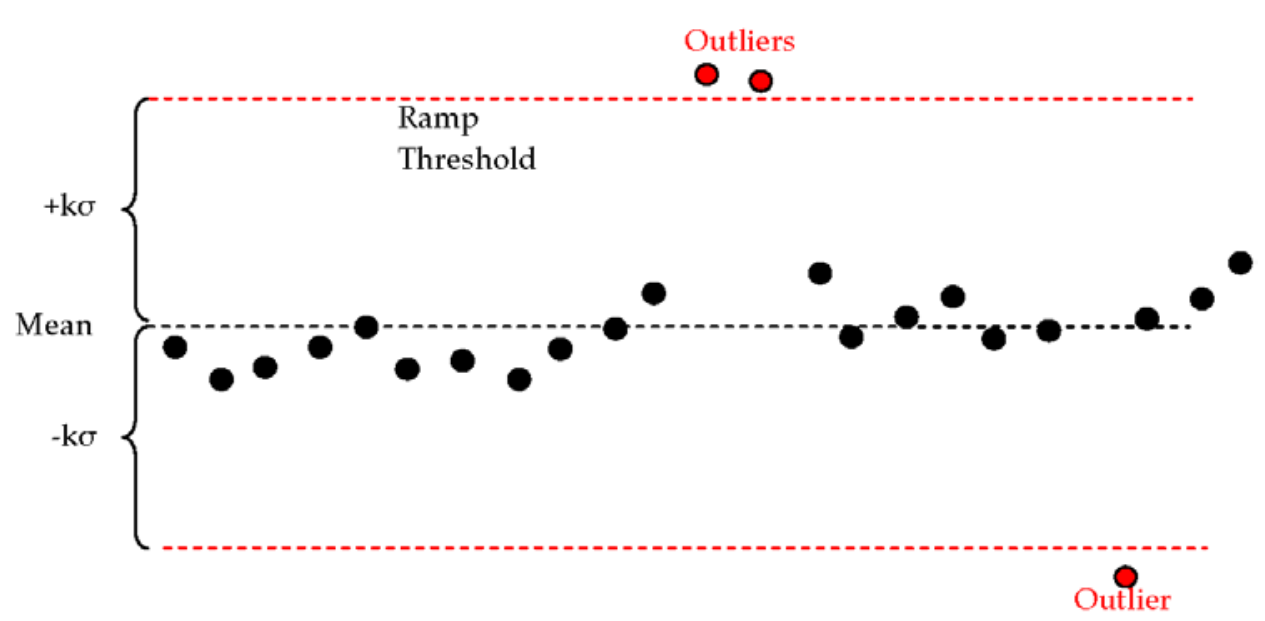

\section{Straight-Line Detection Algorithm}

To replace the missing points, the OSISoft ${ }^{\circledR}$ PI database uses an interpolation (connecting the beginning and the end of the region with missing data by a line). These points should be excluded from the statistical analysis (Figure 40).

Point $x_{t}$ does not belong to time series $X$, if

$$
x_{t}-x_{t-1}=x_{t-1}-x_{t-2}=x_{t-2}-x_{t-3}
$$

Figure 40. Straight-Line Detection

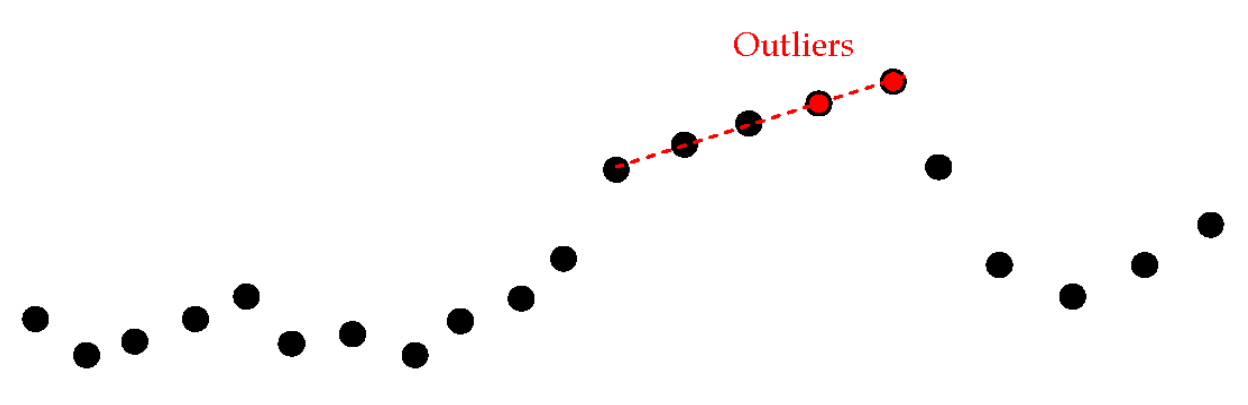




\section{Software Tool Design and User Interface}

Microsoft Visual Studio ${ }^{\circledR} 2008$ was used to develop the regulation prediction tool, which is deployed on the Microsoft Windows ${ }^{\circledR}$ platform and Microsoft ${ }^{\circledR}$.NET Framework. The prototype consists of three main modules: the database, the uncertainty evaluation module and the display for results and alerts. The database is implemented in Oracle ${ }^{\circledR} 10 \mathrm{~g}$. Examples of the software user interface are presented in Figure 41 - Figure 44.

\section{Main User Interface Screen}

The user can select any required date and time to display information on the forecasted and actual parameters in tabular or graphical form. This information includes:

- load forecast

- wind Generation forecast

- solar generation forecast

- interchange schedule

- generation requirements forecast (Load forecast minus wind \& solar generation forecasts and minus interchange schedule)

- generation schedule

- actual load

- actual wind generation

- actual solar generation

- actual interchange

- actual generation requirements

- available generation ramping capability.

The user can select the desired uncertainty ranges with any required level of confidence. The ranges reflect the uncertainty in generation requirements caused by different sources: wind, load, etc.

Screenshots shown in Figure 41-Figure 42 are examples of the capacity requirements displays. Figure 43 shows an example of ramping requirements display.

The software tool can display capacity and ramping requirements for three look-ahead horizons (modes):

1. Day-ahead mode (next day, 24-h, 1-h resolution). Figure 41 shows a screen shot of the dayahead display.

2. Real-time mode (65 $\mathrm{min}$ ahead, 5 -min resolution). Figure 42 shows a screen shot of the realtime display.

3. Hour-ahead mode (up to $5 \mathrm{~h}$ ahead, 15-min resolution). 
Figure 41: Screenshot of Day-Ahead Capacity Requirements Display

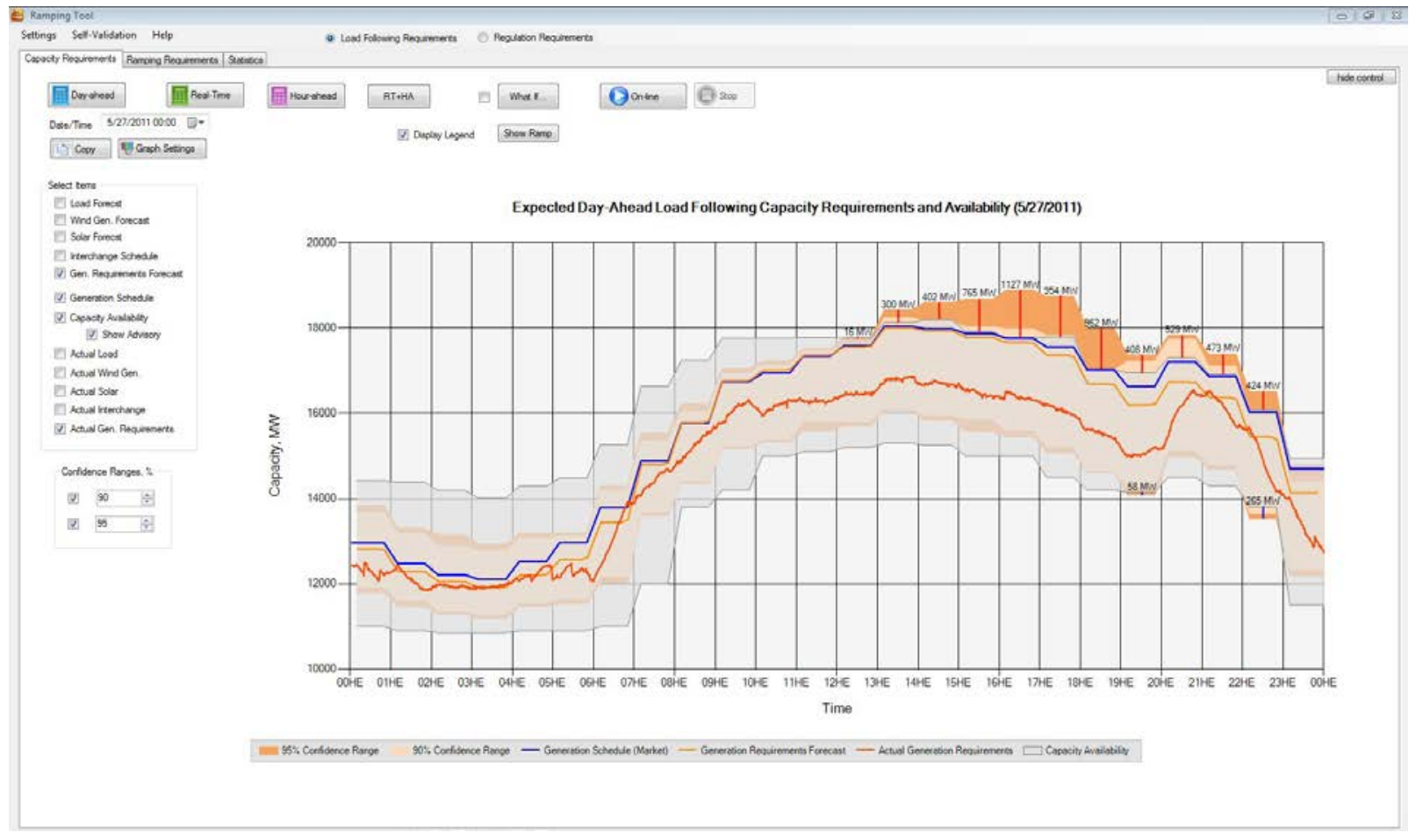

Figure 42: Screenshot of Real-Time Requirements Display

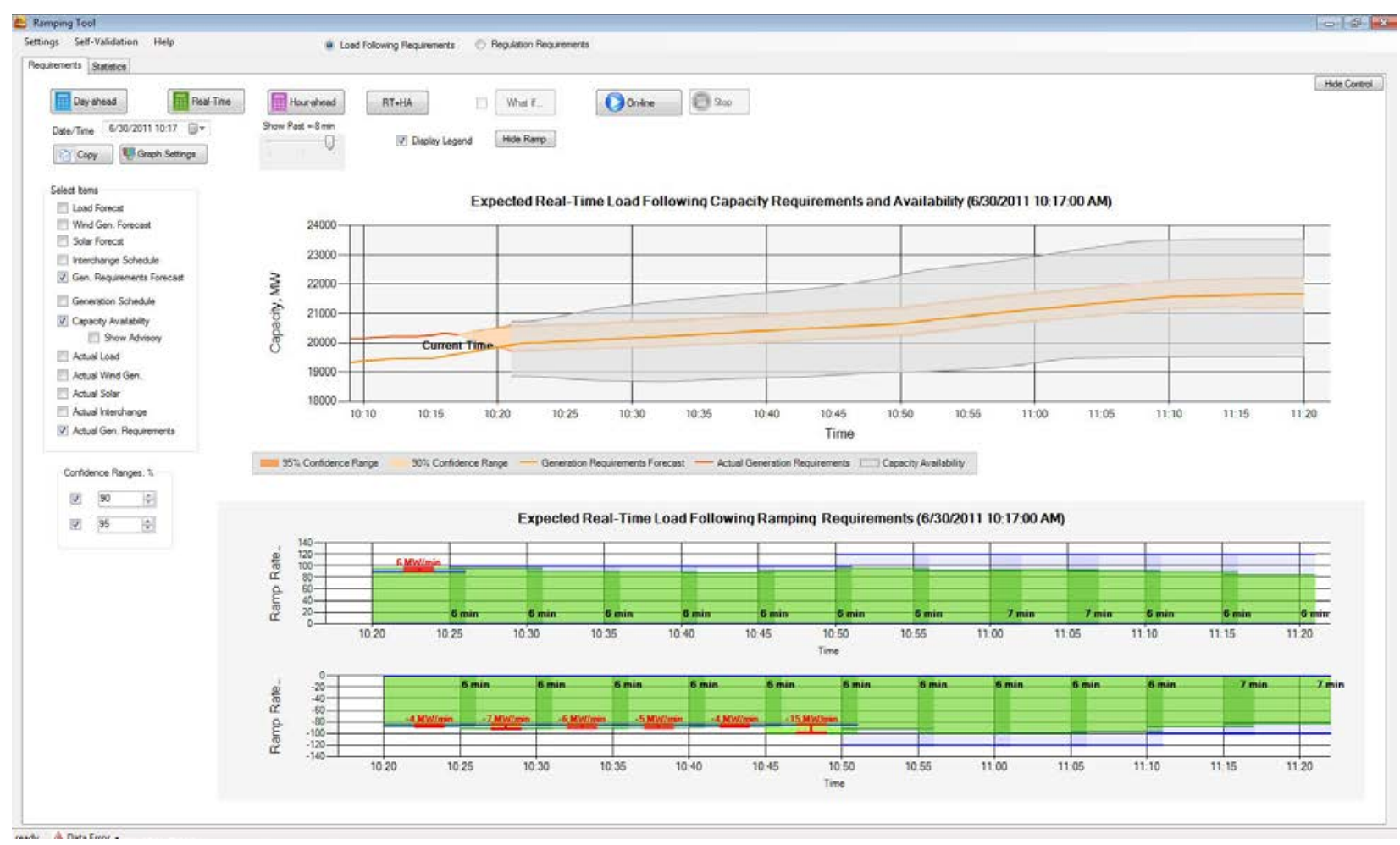


Figure 43: Screenshot of Day-Ahead Ramping Requirements Display

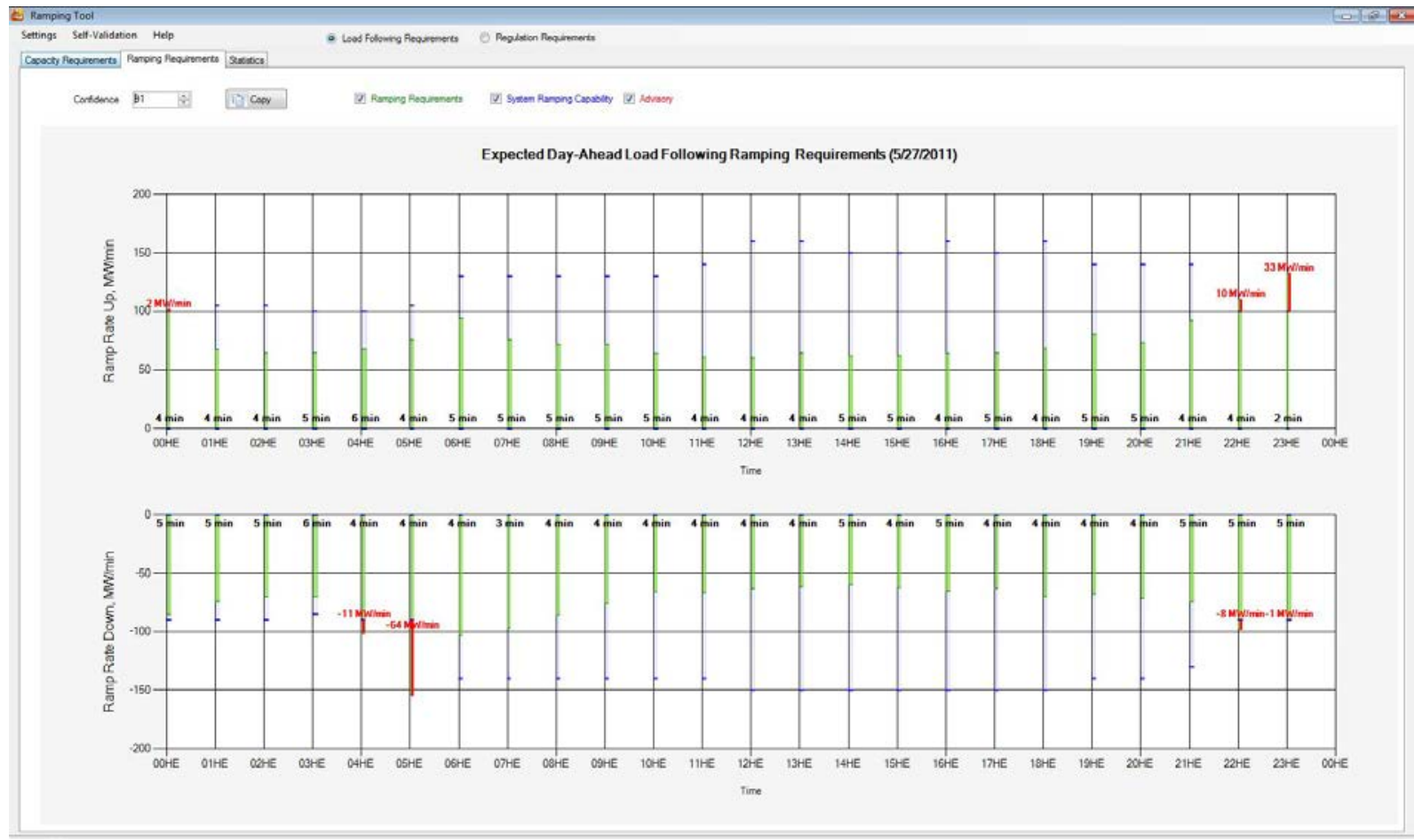

The statistical analysis display is shown in Figure 44. Using this tool, one can analyze statistical characteristics of forecast errors and plot different histograms and CDF functions.

Figure 44: Screenshot of Statistical Analysis Display

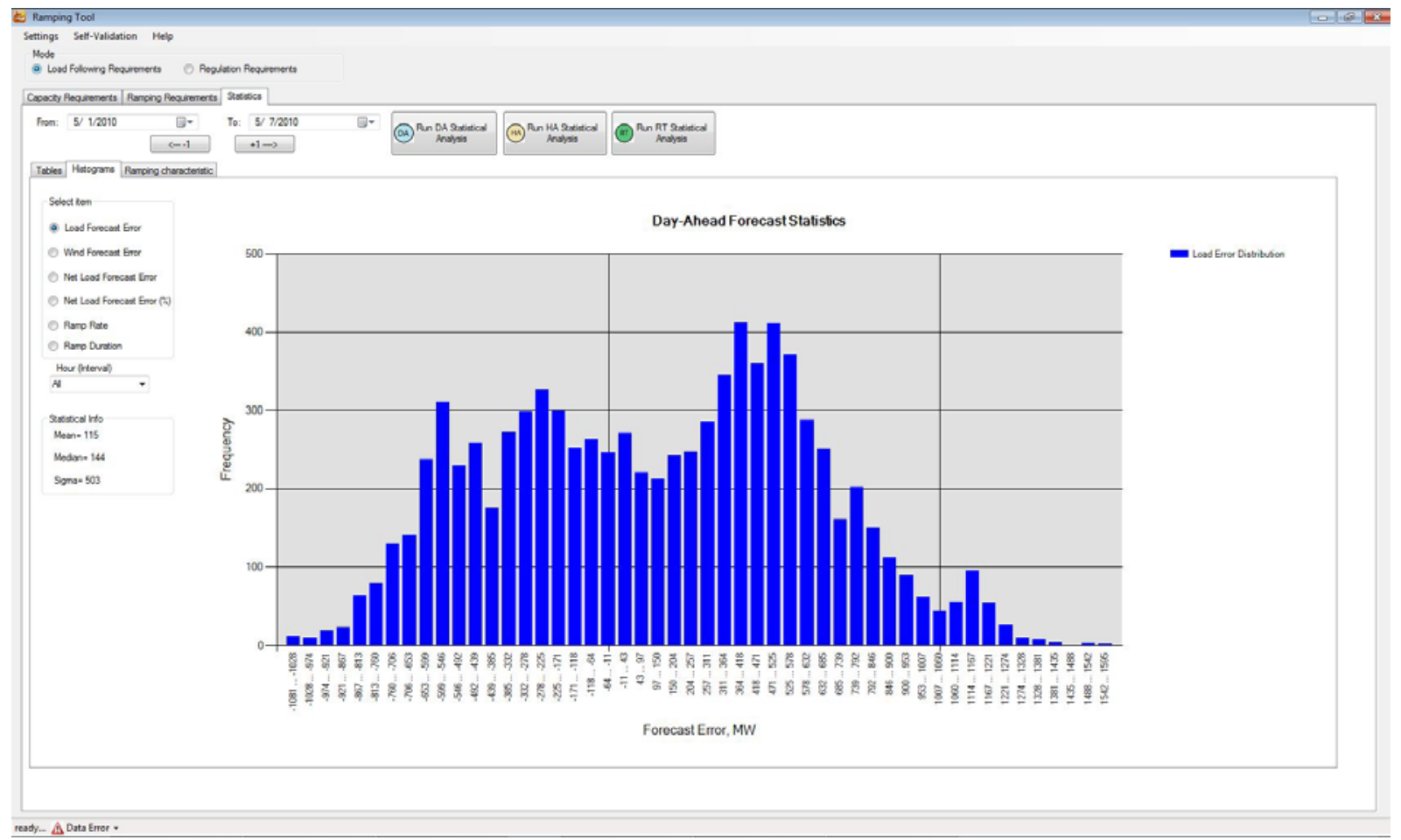




\section{Test Plan for the Ramp Tool Prototype}

A comprehensive plan to test the tool prior to release has been developed. It includes functional, requirements and performance testing of the database, database loading, analytical, and graphical user interface (GUI).

A server will be initialized by installing the test database with no data. It will then be updated using the data loading software and examined for correct loading. One or more standard personal computer workstations, configured with needed supporting software, in accordance with the Prototype Hardware and Software Requirements and Prototype Data Specification, will then be loaded and tested using procedures in the test tool.

Testing is performed using the database running on a separate workstation as a server and with the database running on the local workstation.

Items and features to be tested:

- Tool analytics test

- Tool GUI.

- Display graphical information

Verify that the GUI provides an analysis screen for selected data sets for a specified duration, and that this analysis screen includes a line graph of selected data. The user must be able to select any required date and time for which to display information in the graphical form on the forecasted and actual parameters.

o Uncertainty prediction (confidence bands) display test

Verify that the user is able to select the desired uncertainty ranges with any required level of confidence. The uncertainty ranges reflect the uncertainty in generation requirements caused by different sources such as wind, load, etc. The uncertainty prediction screen includes:

- capacity requirements

- ramping requirements.

- Advisory display test

The tool should display available generation capability in the system and notify of potential deficiency of balancing resources in the system. The following advisories must be provided:

- deficiency of capacity

- deficiency of ramping capability. 
- Oracle database performance testing.

o Connection to the Oracle server

Confirm that the software tool connects to the database server through the local network. The user should be able to specify the database internet protocol (IP) address, user name and password.

o Reading data from the Oracle server

Confirm that the software tool can read information from, and save information to, the database.

- Computational test

The purpose of these computation tests is to 1) verify that all calculations performed by the Wind Uncertainty Prediction Tool are consistent with published algorithms and 2) validate that the tool predicts system balancing requirements for the following lookahead horizons:

o $65 \mathrm{~min}$ ahead (Real-Time mode)

o 4-7 h ahead (Real-Time + Hour-Ahead mode)

o next day (Day-Ahead mode)

- All user controls

Validate functionality of miscellaneous user controls:

o graph settings screen

o "Copy to clipboard" feature

o "What-If" screen

0 on/off control for display of legend on graph

- Statistical analysis module test

To validate the statistical analysis module developed by PNNL using Visual Studio ${ }^{\circledR}$ 2008 Integrated Development Environment (IDE), results produced by this module should be compared with standard (etalon) results obtained from a professional statistical package. The MATLAB ${ }^{\circledR}$ statistical toolbox is used as the standard statistical package.

- On-line mode test

In on-line mode, the tool should use the current date and time. The tool updates the predicted capacity and ramping requirements every minute automatically. The tool also updates displayed actual and forecasted information every minute. Thus, the user will always see the latest available information in the system.

- Acceptance test (based on self-validation procedure)

To validate the accuracy of the uncertainty prediction model, a self-validation algorithm is used. The self-validation algorithm is based on comparing the predicted uncertainty 
ranges against the actual observed values for the same dispatch intervals. The algorithm counts how many points belong to the predicted intervals with a specified confidence level, and calculates the percentage of points found within the intervals. The uncertainty algorithm is validated if the calculated percentages are close to the targeted percentages. Self-validation tests must be performed for:

o Real-time mode

o Hour-ahead mode

o Day-ahead mode. 


\section{CHAPTER 5 Simulation Results}

Using the prototype tool, case studies have been run to test the uncertainty assessment approach and to demonstrate the capabilities of the tool. CAISO's actual data were used in the simulation and the tool development. The actual data used include total load, total wind generation, load forecast (day-ahead, hour-ahead and real-time forecast), and wind generation forecast.

Figure 45 shows the day-ahead uncertainty ranges for the system net load. The light and dark orange areas represent the evaluated capacity ranges for the $90 \%$ and $95 \%$ confidence levels. The blue curve is the day-ahead generation schedule. The gray area is the available balancing reserve, which is calculated from the margin of online generators. The system would have adequate balancing reserve with a specified confidence level if the available balancing reserve (the gray area) covers the entire net load uncertainty range (the dark or light orange area). Otherwise, deficiency of balancing reserve occurs; in this case, there is a certain probability that the online generation will not be able to follow the net load requirement. The deficiency of the balancing reserve is shown using the red (generation up) and blue (generation down) bars in Figure 45. It gives an indication that it is necessary to commit or de-commit additional generators to achieve the desired confidence level for the balancing reserve.

Figure 45: Day-Ahead Capacity Prediction

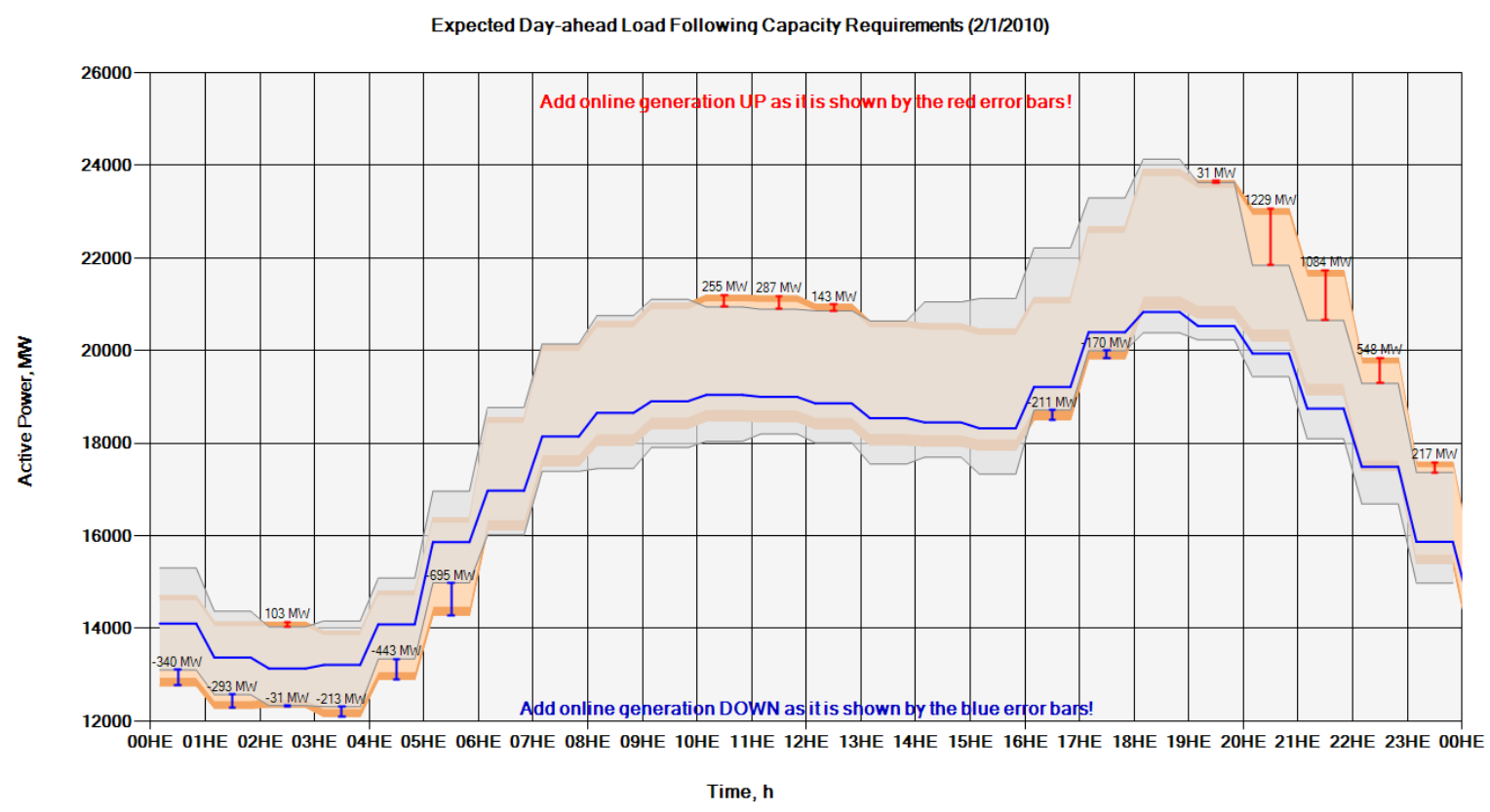


Figure 46 shows the results of the evaluation of hourly day-ahead ramping requirements. The length of each green bar indicates the ramp rate requirements $(\mathrm{MW} / \mathrm{min})$. The width of each bar indicates the ramp duration requirements (minutes). From this figure, operators can compare the ramping requirements against available system ramping capacity to see whether the system has sufficient resources to meet the ramp rate requirements. Details of the methodology for estimating system ramping capability are given in Appendix A.

Figure 46: Day-Ahead Ramping Requirements Prediction

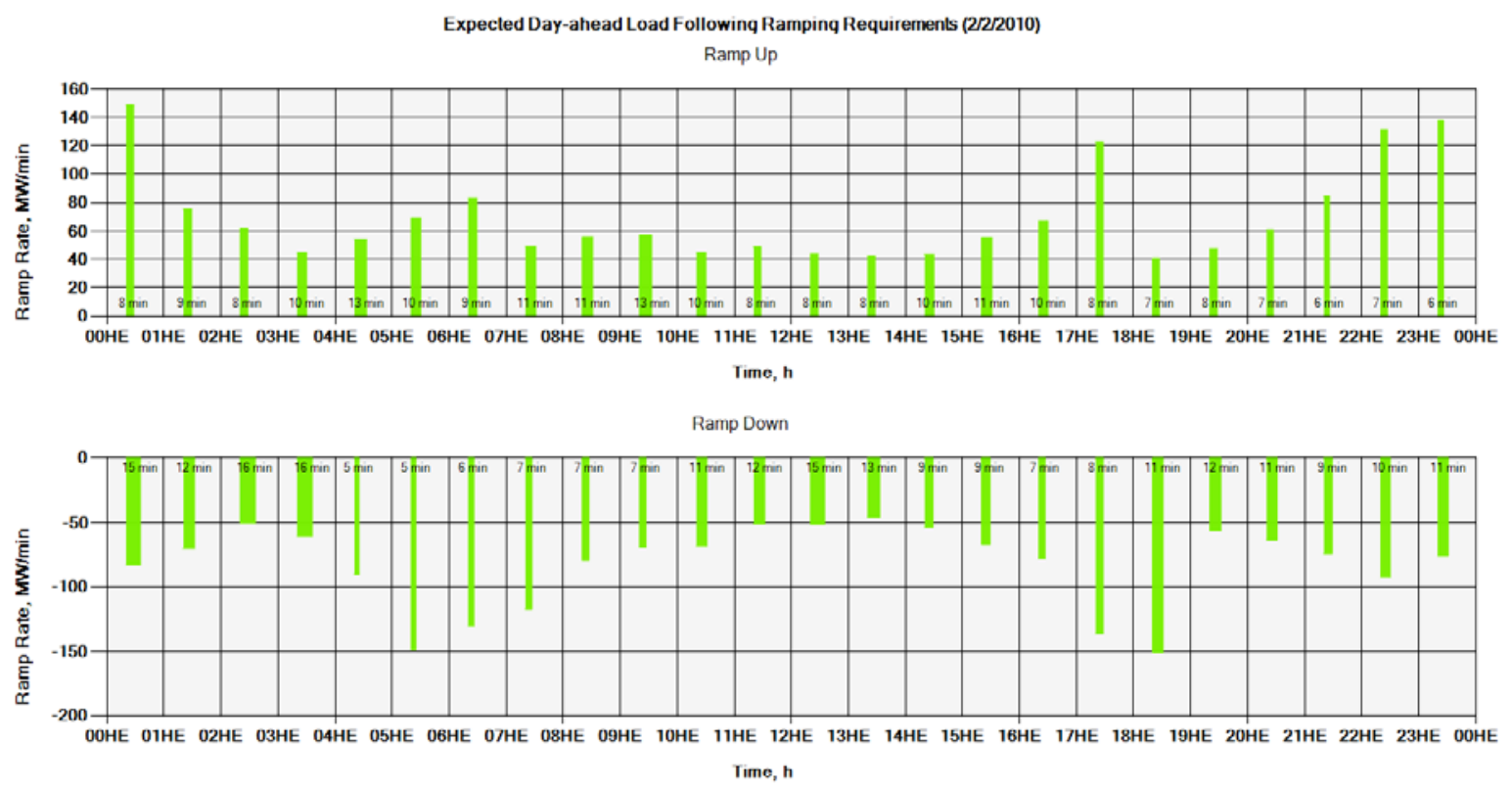

Figure 47 presents an example snapshot of the hour-ahead capacity requirements for a 7-hour time horizon. Similar to Figure 45, the light and dark orange areas represent the evaluated capacity ranges for the $90 \%$ and $95 \%$ confidence levels. The blue curve is the hour-ahead generation schedule. Also similar to Figure 45, the deficiency of the balancing reserve, shown as red (generation up) and blue (generation down) bars, provides guidance to operators for committing or de-committing additional generators to achieve the desired confidence level for the balancing reserve.

Figure 48 presents the hour-ahead ramping requirements for a 7-hour time horizon. This evaluation is based on statistical analysis of hour-ahead forecast uncertainty. The length of each green bar indicates the ramp rate requirements $(\mathrm{MW} / \mathrm{min})$. The width of a bar indicates the ramp duration requirements (minutes). This information can be used to determine whether the system would be able to meet the ramp rate requirements. 
Figure 47: Hour-Ahead Capacity Requirements Prediction

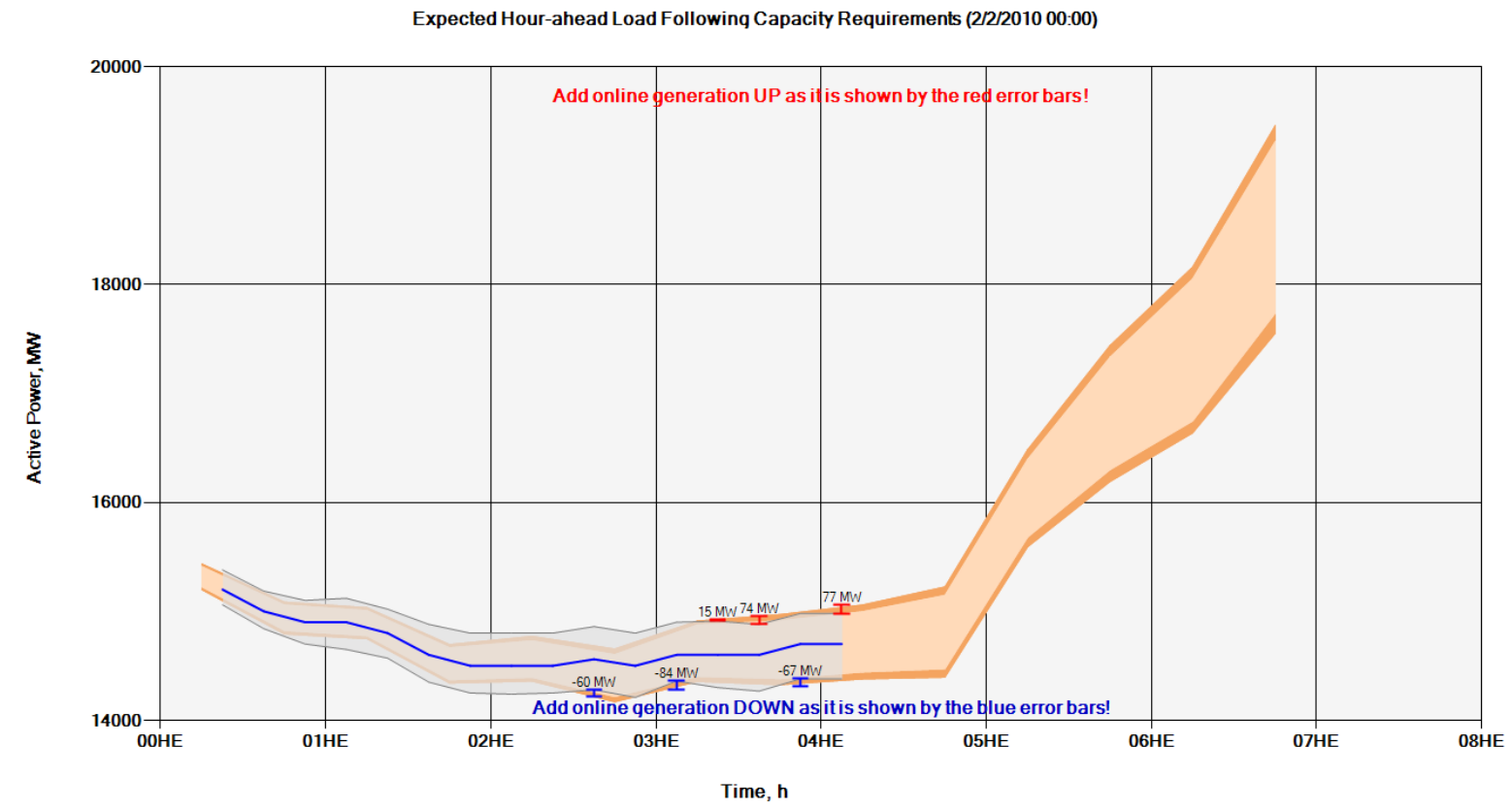

Figure 48: Hour-Ahead Ramping Requirements Prediction
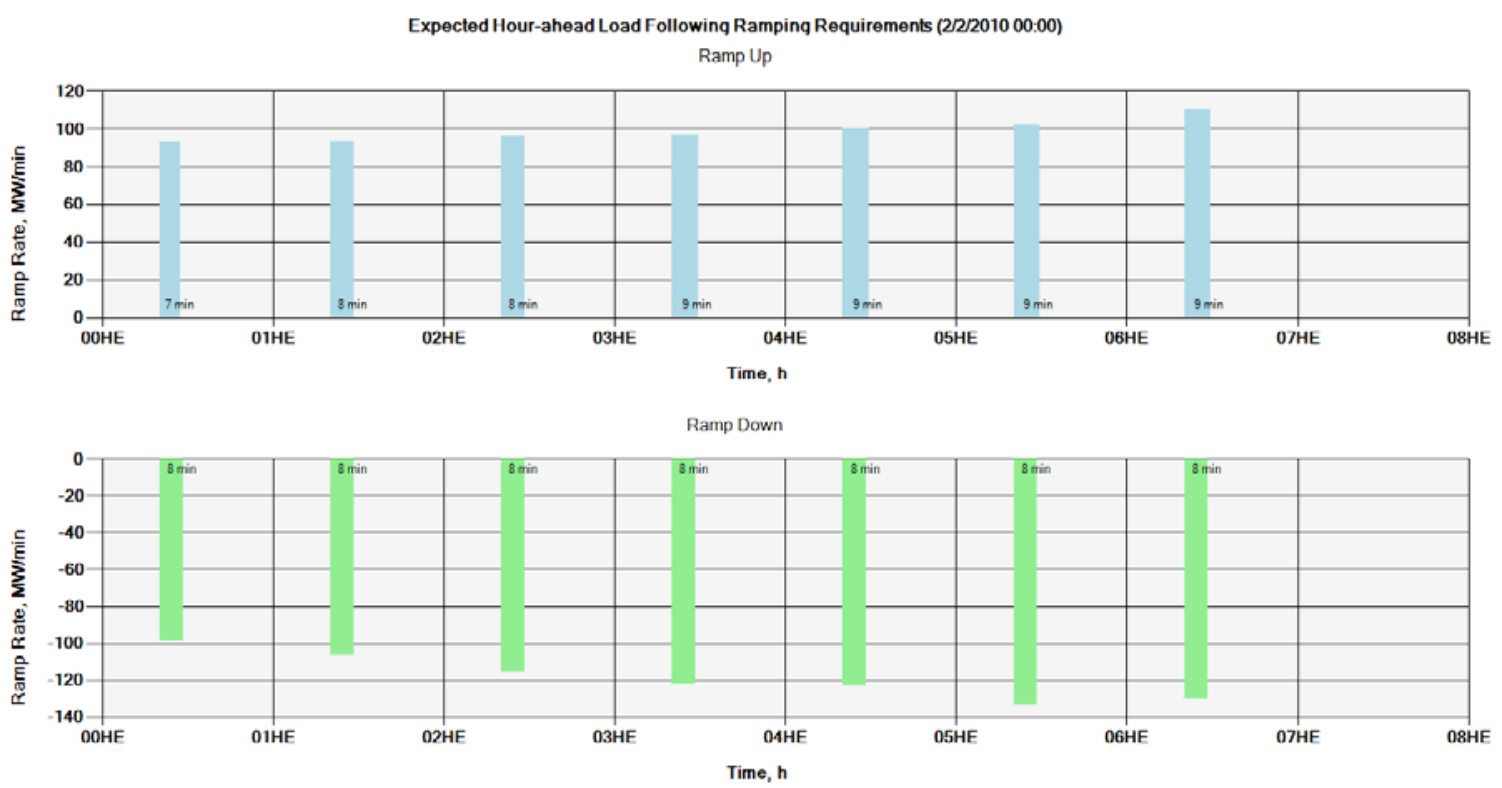

\section{Self-Validation Results}

To validate the accuracy of the uncertainty evaluation model, a self-validation test was performed. CAISO's actual statistical information for years 2010-2011 was used in this test. 


\section{Real-Time Mode}

Results of real-time mode self-validation for May-June 2011 are presented in Figure 49. One can see that the observed percentage values are very close to the targeted percentage values. For example, the percentage of the actual generation requirements points found within the $98 \%$ confidence interval is about $96.23 \%$, and the percentage within the $95 \%$ confidence interval is about $93.1 \%$. Thus the uncertainty evaluation model tests have confirmed the adequacy of the proposed uncertainty evaluation algorithm as well as the proper operation of the developed prototype tool.

Figure 49: Real-Time Mode Self-Validation Results

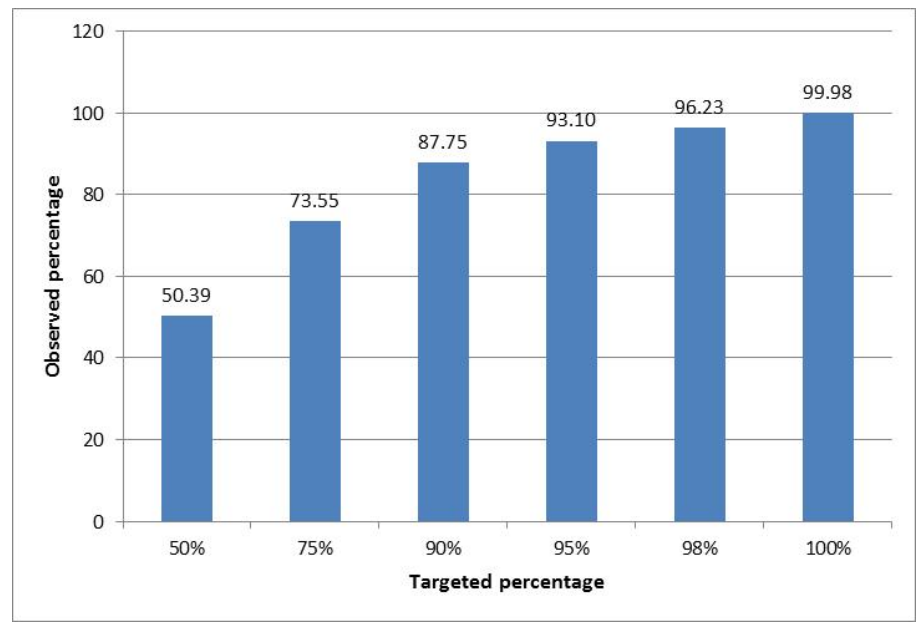

\section{Hour-Ahead Mode}

Results of hour-ahead mode self-validation for May-June 2011 are presented in Figure 50. Similar to real-time mode testing results, we can see that the percentages of observed actual values are very close to the targeted percentage values.

Figure 50: Hour-Ahead Mode Self-Validation Results

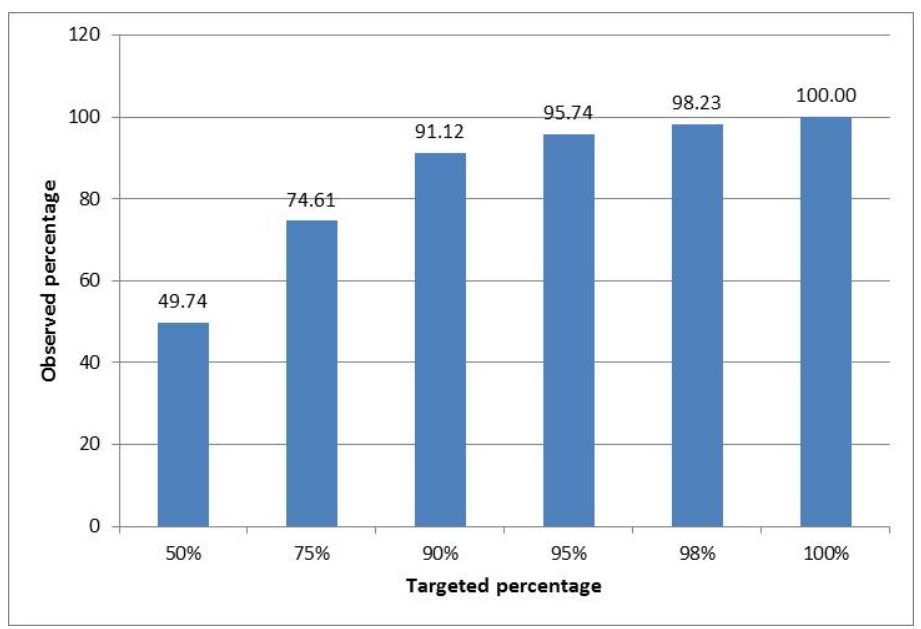




\section{Day-Ahead Mode}

Results of day-ahead mode self-validation for May-June 2011 are presented in Figure 51. One can see that the observed percentage values are very close to the targeted percentage values. Thus, the tests performed have validated the accuracy of the predictions produced by the tool.

Figure 51: Day-Ahead Mode Self-Validation Results

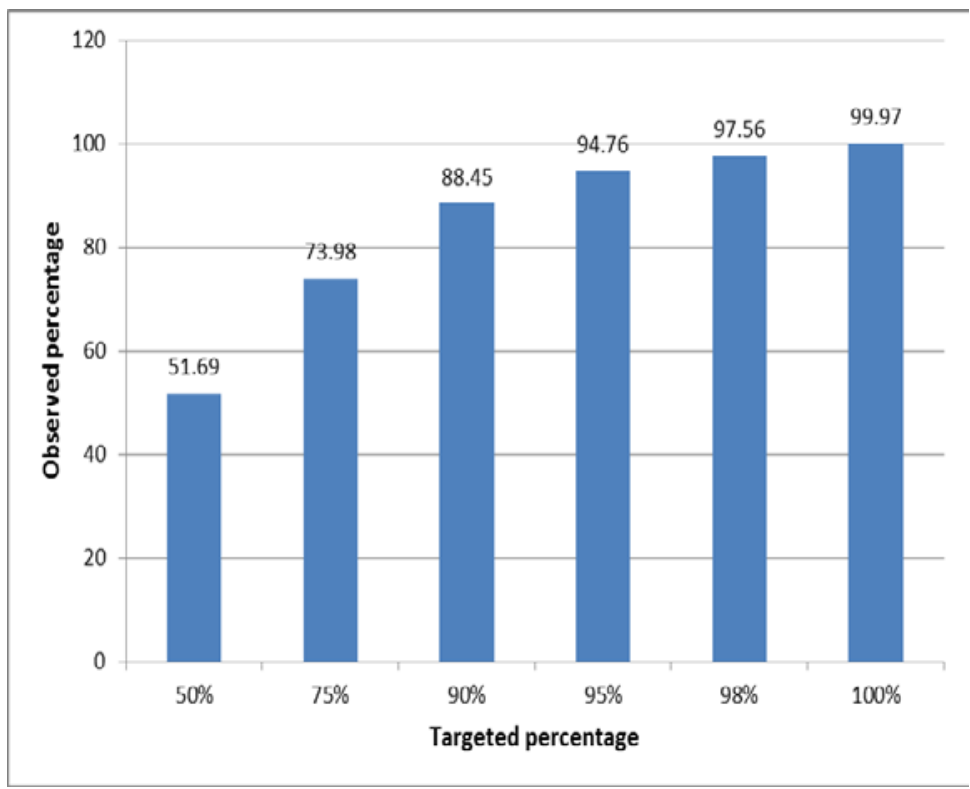

\section{Prediction of Real-Time Market Price Spikes}

Figure 52 presents an example snapshot of the real-time capacity requirements for the next operating hour. The light and dark orange areas represent the evaluated capacity ranges for the $90 \%$ and $95 \%$ confidence levels. The blue curve is the real-time generation schedule (economic dispatch). The gray area is the available balancing reserve, which is calculated from the margin of online generators. The system would have adequate balancing reserve with a specified confidence level if the available balancing reserve (the gray area) covers the entire net load uncertainty range (the dark or light orange area). Otherwise, deficiency of balancing reserve occurs. This means that there is a certain probability that the online generation will not be able to follow the net load requirement.

The deficiency of the balancing reserve, shown as red bars, provides guidance to operators for committing or de-committing additional generators to achieve the desired confidence level for the balancing reserve.

It has been found that the tool is capable of predicting intra-hour deficiency in generation capability. This deficiency of balancing resources can cause price spikes in the real-time market. One can see in Figure 52 that a more than 300 MW deficiency in the system generation capability is predicted in the 10- to 25-min look-ahead period (red error bars). Figure 53 shows 
the CAISO's real-time market price information. Price spikes occurred in the CAISO market at the same time at which generation capability deficiency was predicted by the tool.

Figure 52: Real-Time Capacity Requirements Prediction

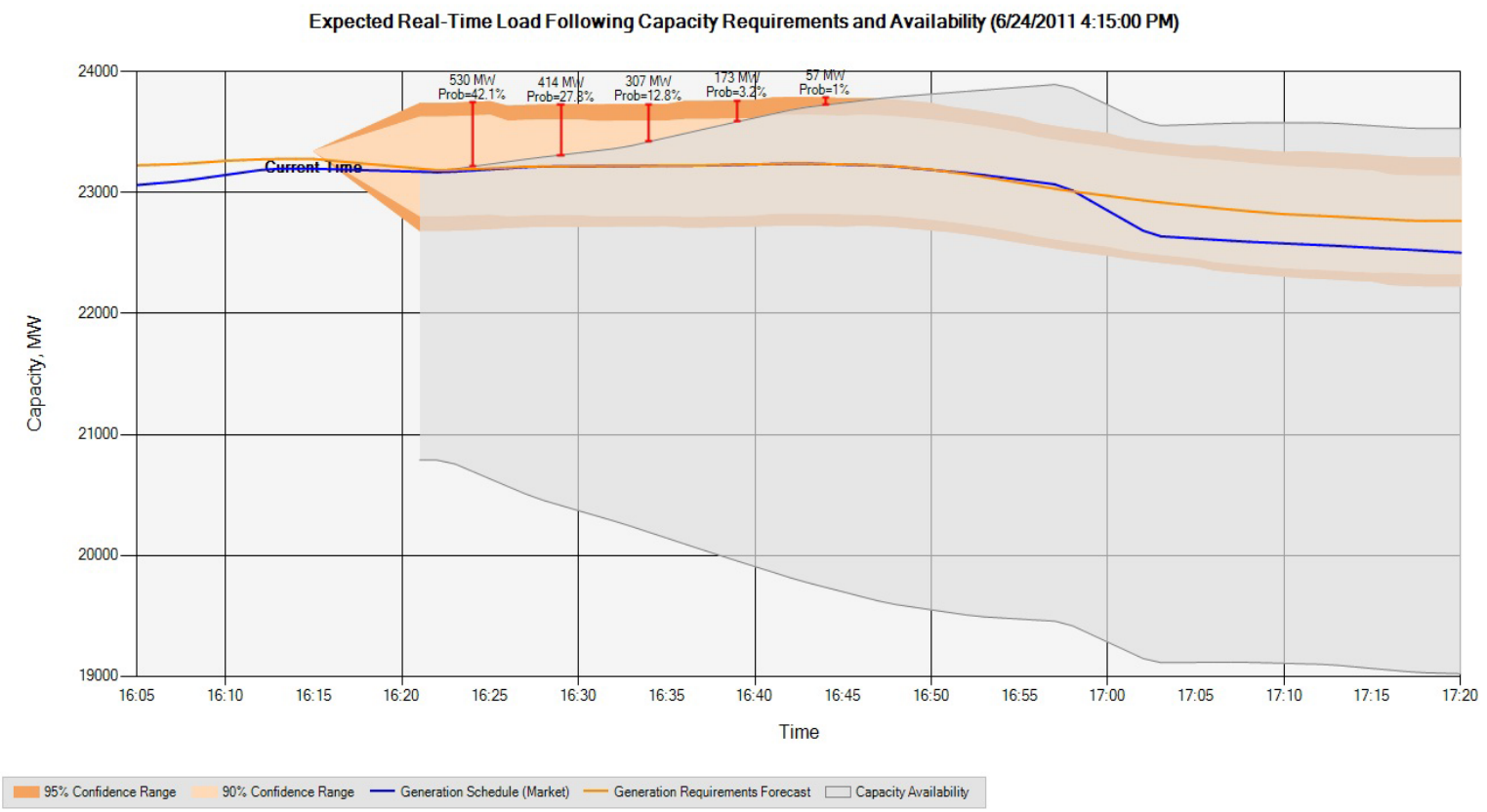

Figure 53: CAISO Real-Time Price*

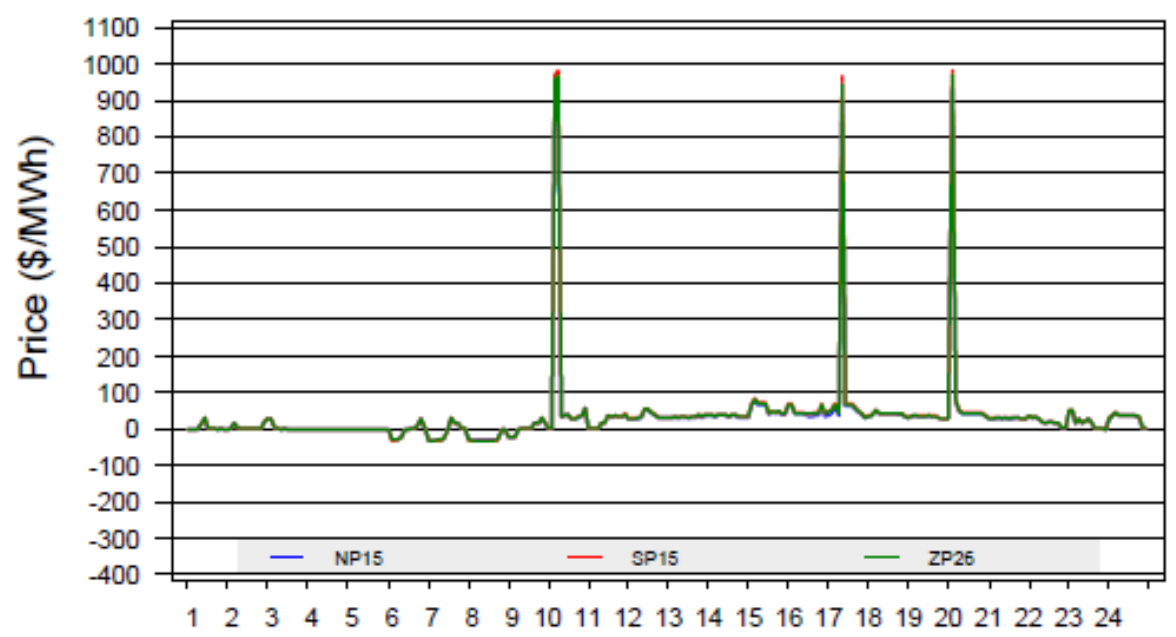

*Available at http://www.caiso.com/Documents/June\%202011/DailyMarketWatch RealTime Jun 24 2011.pdf

\section{Real-Time Ramping Requirements}

Figure 54 presents the real-time ramping requirements prediction display. This evaluation is based on statistical analysis of real-time forecast uncertainty. The length of each green bar 
indicates the ramp rate requirements $(\mathrm{MW} / \mathrm{min})$. The width of each green bar indicates the ramp duration requirements (minutes). This information can be used to determine whether the system would be able to meet the ramp rate requirements. From this display, operators can compare the ramping requirements against available system ramping capacity (blue bars) to see whether the system has sufficient resources to meet the ramp rate requirements. Methodology to estimate system ramping capability is given in Appendix A. Insufficient ramping capability is indicated using red error bars (Figure 54).

Figure 54: Real-Time Ramping Requirements
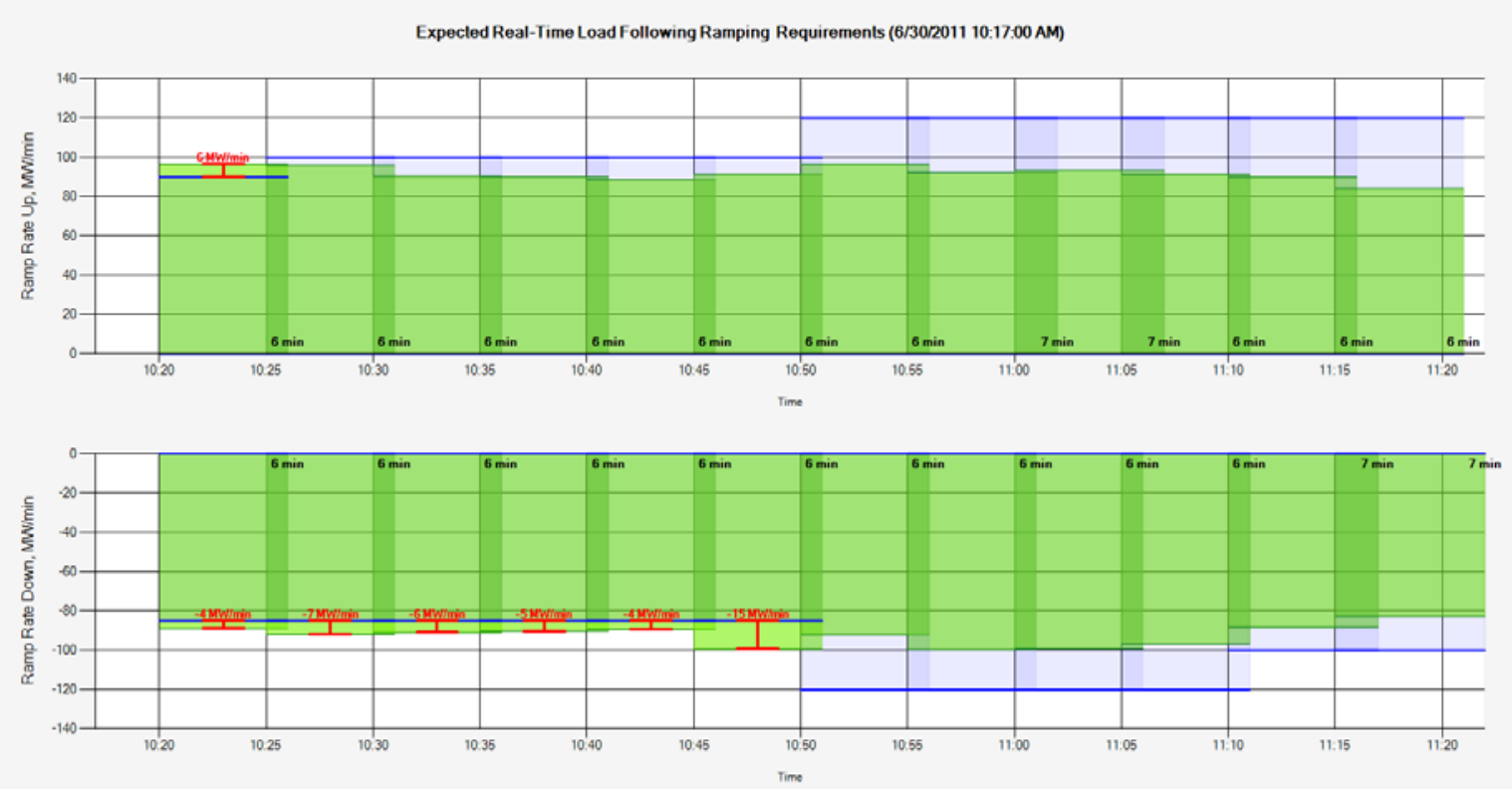


\section{CHAPTER 6: Conclusions}

A methodology capable of evaluating the impact of wind generation and load uncertainties, as well as unexpected generation outages, on balancing resource requirements has been developed, implemented in a software tool and deployed in the operating environment. The tool enables evaluation of the uncertainty ranges for the required generation performance envelope for a look-ahead period. The generation performance envelope includes the required balancing capacity, ramping capability and ramp duration capability.

The development, implementation and deployment includes the following elements:

- Evaluation of the capacity and ramping requirements using a specially developed probabilistic algorithm based on histogram analysis, and incorporating various sources of uncertainties, both continuous (wind and load forecast errors) and discrete (forced generator outages and start-up failures).

- Evaluation of the look-ahead generation performance requirements envelope using a "flying brick" technique for the worst-case scenario within a user-specified confidence level.

- A self-validation algorithm to assess the accuracy of the predicted uncertainty ranges

- A software tool based on the methodology has been developed. The operating practices at CAISO have also been incorporated. The concept of integrating the probabilistic tool into CAISO EMS environments has been developed taking into account current operating practices.

- Simulation studies using actual CAISO data have been performed. The developed software tool passed all tests performed in accordance with the tool testing specification. Study results have shown that the methodology of the generation-requirements evaluation for uncertainty management is quite accurate and efficient.

The tool is installed at a CAISO control center and has been tested by CAISO specialists. The tool received very positive feedback from CAISO. Testing of the tool performed by CAISO specialists has shown that the tool is also capable of predicting price spikes in the real-time market caused by deficiency of available on-line balancing resources in the system. CAISO expressed its interest in incorporating the tool into CAISO market and scheduling procedures.

\section{Next Steps (Technical Area)}

The proposed steps in the technical area will increase the robustness and performance of the tool while handling the information typically available in control centers, improving the probabilistic models to further reduce predicting range requirements, and adding additional model components reflecting new sources of uncertainty. 
The following specific steps are suggested:

- Integrate the tool with the wind/solar forecast service providers' information to improve the accuracy of the balancing requirements prediction.

- Develop proactive integration methodology to incorporate the tool into CAISO market and unit commitment procedures.

- Develop and implement a new generation of statistical methods to address nonstationary characteristics of forecast errors. Further improve the accuracy and robustness of the tool.

- Perform probabilistic modeling of uninstructed deviation of generating units.

- Develop a decision support system using the tool to help dispatchers addressing potential issues of the system balancing process.

- Incorporate consideration of the new BAAL standard into balancing requirements assessment methodology.

- Address data interpretation and quality issues to enhance robustness of the tool.

\section{Next Steps (Commercialization)}

The purposes of the planned commercialization activities include a wide dissemination and technology transfer effort with the ultimate objective to install the tool in several control centers in California. Among these activities the highest priority tasks will include a proactive integration of the software tool in a CAISO control center, its comprehensive testing and final adjustments. The results of this effort will create a platform for a wider reach in California by serving as an example for other control areas and utilities in the state. In parallel with the California activities, the results of this project will be widely distributed via Web seminars organized for the industry and by presenting them at the industry forums, including Utility Wind Integration Group, WECC groups, NERC subcommittees, and conferences. It is expected that through these activities (supported by DOE), the impact of this project will be extended from the statewide level to the nationwide level. 


\section{CHAPTER 7: References}

CAISO - California Independent System Operator. 2006. “Market Redesign and Technology Update Tutorial for Market Participants," CAISO, Folsom California.

CAISO - California Independent System Operator. 2007. "Integration of Renewable Resources Report," CAISO, November 2007. [Online.] Available: http://www.caiso.com/1ca5/1ca5a7a026270.pdf.

Constantinescu, E. M., V. M. Zavala, M. Rocklin, S. Lee, and M. Anitescu. 2011. “A computational framework for uncertainty quantification and stochastic optimization in unit commitment with wind power generation." IEEE Trans. Power Syst. 26(1):431-441.

Energy \& Meteo Systems. 2007. “Wind power prediction Previento,” Energy \& Meteo Systems. [Available online]: http://energymeteo.de/media/PrevientoENG.pdf .

Kariniotakis, G. 2008. “ANEMOS, leading European Union research on wind power forecasting." In Proc. International Wind Forecast Techniques and Methodologies Workshop, July 24-25, 2008. [Available online]:

http://www.bpa.gov/corporate/business/innovation/docs/2008/BPA_California\%20ISO\% 20ANEMOS\%20Presentation.pdf.

Kehler, J., M. Hu, M. McMullen, and J. Blatchford. 2010. “ISO perspective and experience with integrating wind power forecasts into operations." In Proc. IEEE General Meeting, July 25-29, 2010, Minneapolis, Minnesota.

Lerner, J., M. Grundmeyer, and M. Garvert. 2009. “The role of wind forecasting in the successful integration and management of an intermittent energy source." Energy Central, Wind Power, vol. 3, no. 8, July 2009.

Luig, A., S. Bofinger, and H. G. Beyer. 2001. "Analysis of confidence intervals for the prediction of the regional wind power output." In Proc. 2001 European Wind Energy Conference, July 2-6, 2001, Copenhagen, Denmark.

Maggio, D., C. D’Annunzio, S.-H. Huang, and C. Thompson. 2010. “Utilization of forecasts for wind-powered generation resources in ERCOT operations." In Proc. IEEE PES General Meeting, July 25-29, 2010, Minneapolis, Minnesota.

Makarov, Y. V., C. Loutan, J. Ma, and P. De Mello. 2009. “Operational Impacts of Wind Generation on California Power Systems.” IEEE Trans. on Power Syst. 24:1039-1050.

Makarov, Y., P. Etingov, J. Ma, Z. Huang, and K. Subbarao. 2011. “Incorporating Uncertainty of Wind Power Generation Forecast into Power System Operation, Dispatch, and Unit Commitment Procedures." IEEE Transactions on Sustainable Energy 2(4):433-442. 
MathWorks $^{\circledR}$. 2010. “MATLAB Statistical toolbox,” User's Guide. The MathWorks, Inc, Natick, Massachusetts.

Pinson, P., G. Kariniotakis, H. A. Nielsen, T. S. Nielsen, and H. Madsen. 2006. "Properties of quantile and interval forecasts of wind generation and their evaluation." In Proc. European Wind Energy Conference E Exhibition, February 2-March 2, 2006, Athens, Greece.

Ummels, B. C., M. Gibescu, E. Pelgrum, W. L. Kling, and A. J. Brand. 2007. “Impacts of Wind Power on Thermal Generation Unit Commitment and Dispatch." IEEE Trans. Energy Conversion 22(1):44-51.

Wang, J., M. Shahidehpour, and Z. Li. 2008. “Security-constrained unit commitment with volatile wind power generation." IEEE Trans. Power Syst. 23(3):1319-1327.

WECC - Western Electricity Coordinating Council. 2007. “WECC Standard BAL-STD-002-0 Operating Reserves." [Online.] Available: http://www.nerc.com/files/BAL-STD-002$0 . p d f$.

WSCC - Western Systems Coordinating Council. 1988. “WSCC Operating Reserve White Paper." [Online.] Available: http://www.wecc.biz/library/Library/White\%20Papers/Operating\%20Reserves.pdf.

Zack, J. 2006. "An analysis of the errors and uncertainty in wind power production forecasts." In Proc. WINDPOWER Conference and Exhibition 2006, June 4-7, 2006, Pittsburgh, Pennsylvania. 


\section{CHAPTER 8: Glossary}

AGC

ALFS

AS

BA

BAAL

CAISO

$\mathrm{CDF}$

CEC

COPT

DA

DAM

DOT

ED

EFDH

EFORd

EMS

$\mathrm{FOH}$

FOP

FOR

FORd

GADS

GUI

HASP

ISO

MTTF

MTTR

MW

NERC

PDF

PIER automatic generation control

adaptive load forecasting system

ancillary services

balancing authority

Balancing Authority Area Control Error Limit

California Independent System Operator

cumulative distribution function

California Energy Commission

capacity outage probability table

day ahead

day-ahead market

dispatch operating target

economic dispatch

equivalent forced derating hours

equivalent forced outage rate demand

energy management system

forced outage duration within a year, (hours)

full outage probability

forced outage rate

forced outage rate demand

Generating Availability Data System

graphical user interface

hour-ahead scheduling process

Independent System Operator

mean time to failure

mean time to repair

megawatt

North American Electric Reliability Corporation

probability density function

Public Interest Energy Research 
PNNL

RD\&D

REG

RT

RTED

RTM

RTUC

SCADA

SCUC

$\mathrm{SH}$

STUC

TND

$\mathrm{UC}$

UWIG

WECC
Pacific Northwest National Laboratory

research, development, and demonstration

regulation

real time

real-time economic dispatch

real-time market

real-time unit commitment

supervisory control and data acquisition

security-constrained unit commitment

service hours

short-term unit commitment

truncated normal distribution

unit commitment

Utility Wind Integration Group

Western Electricity Coordinating Council 


\section{APPENDIX A: \\ Generation Fleet Ramping Capability}

Each generating unit has specific physical characteristics:

- Minimum generation $-P_{\min }(\mathrm{MW})$

- Maximum generation $-P_{\max }(\mathrm{MW})$

- Maximum ramp rate $-R(\mathrm{MW} / \mathrm{min})$

Those characteristics can be segmented depending on incremental blocks of power output provided by them.

Other characteristics are:

- $\quad$ Scheduled operating point - $P_{\text {sched }}(\mathrm{MW})$

- Regulation reserve (upward and downward) - $P_{R E G_{-} U P,} P_{R E G_{-} D N}(\mathrm{MW})$

- $\quad$ Contingency reserve $-P_{\text {cont }}(\mathrm{MW})$

A simplified ramping characteristic of a generation unit is shown in Figure A.1. Some units can have segments with different maximum ramp rates, but for simplicity the linearized ramping characteristic is used in Figure A.1.

Figure A.1: Generation Unit Ramping Characteristic

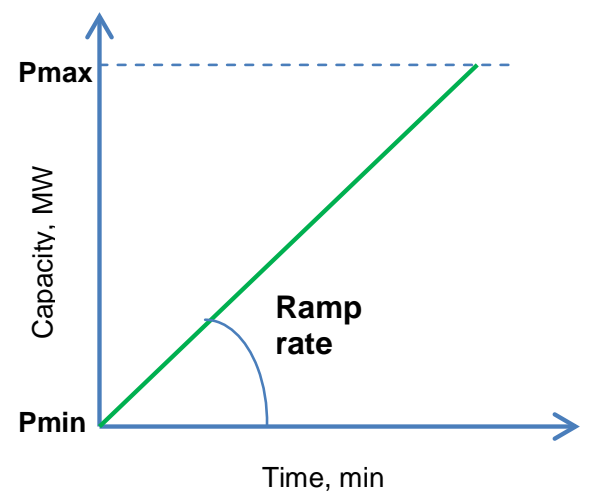

The ramping capability characteristic of unit $i$ at time $t$ starting from an operating point $P_{\text {sched, } i \text { is }}$ as follows:

$$
\begin{aligned}
& P_{\mathrm{UP}, t}^{i}=P_{\text {sched }, i}+R_{i} \cdot t, \\
& P_{\mathrm{DN}, t}^{i}=P_{\mathrm{sched}, i}-R_{i} \cdot t, \\
& t=0 \ldots . . T,
\end{aligned}
$$


where $P_{U P, t}^{i}$ is the maximum available upward capacity of the unit $i$ at time $t ; P_{D N, t}^{i}$ is the maximum available downward capacity of the unit $i$ at time $t$, and $T$ is the duration of the ramping characteristic.

The upward and downward capacity is also limited by the following constraints:

$$
\begin{aligned}
& P_{\mathrm{vP}, t}^{i} \leq P_{\max , i}, \\
& P_{\mathrm{DN}, t}^{i} \geq P_{\min , i}
\end{aligned}
$$

Figure A.2 shows an illustration of the simplified unit ramping capability characteristic. Assume that initially the unit is operated at the scheduled generation point $P_{\text {sched. }}$ The unit cannot change the generation with the ramp rate faster than its maximum ramp rate, $R$. The maximum upward and downward capability of the generation unit is shown using green lines in Figure A.2. The maximum and minimum generation of the unit is also limited by the $P_{\min }$ and $P_{\max }$ constraints (dotted lines in Figure A.2).

Figure A.2: Generation Unit Ramping Capability

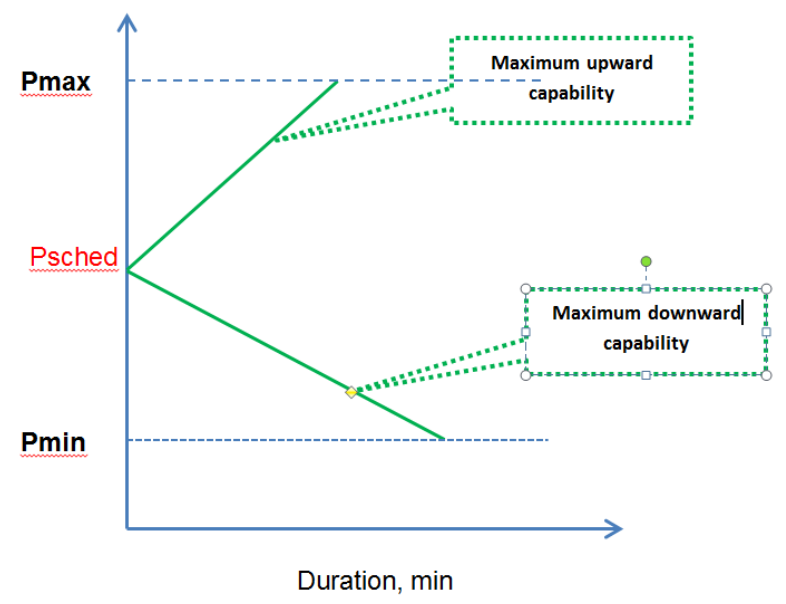

\section{Ramping Capability of the Generation Fleet}

The individual ramping capabilities of generation units should be combined to calculate the total system ramping capability of the online units:

$$
\begin{aligned}
& P_{U P_{\Sigma}, t}=\sum_{i=1}^{N} P_{U P, t}^{i}, \\
& P_{D N_{\Sigma}, t}=\sum_{i=1}^{N} P_{D N, t}^{i},
\end{aligned}
$$

where $\mathrm{N}$ is the number of online units available in the system. 
Figure A.3 shows an illustration of generation fleet ramping capability in terms of its changing capacity. To calculate the maximum available system capability in terms of time and ramp rate, the following equation can be used:

$$
\begin{aligned}
& R_{U P_{\Sigma}, t}=\frac{P_{U P_{\Sigma}, t}}{t}, \\
& R_{D N_{\Sigma}, t}=\frac{P_{D N_{\Sigma}, t}}{t}, \\
& t=1 \ldots T
\end{aligned}
$$

Figure A.3: Illustration of Generation Fleet Ramping Capability (capacity vs. duration)

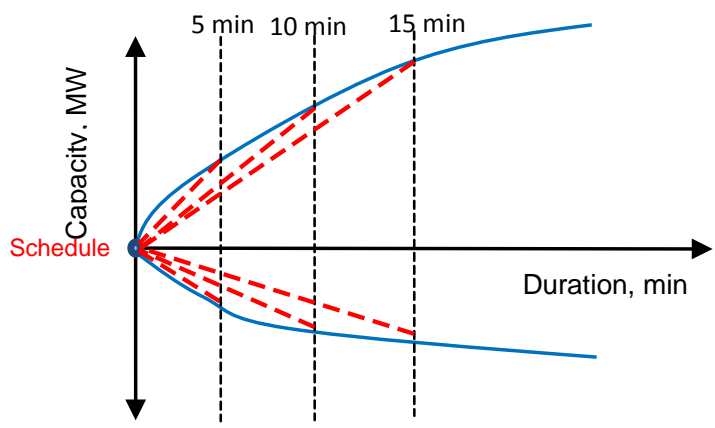

An illustration of the relationship between generation fleet ramp rate capability and maximum ramp duration capability is presented in Figure A.4. Five-minute intervals for the ramp duration are used in this example. During first 5-minute interval, the maximum generation fleet ramp rate capability cannot be higher than $R_{5}$ (Figure A.4). After 5 minutes, the fastest units achieve their maximum capacity limit and maximum system ramp rate capability starts decreasing. Thus, for 10- and 15-minute ramp duration intervals, the generation fleet ramping capability is limited by $R_{10}$ and $R_{15}$, correspondently (Figure A.4).

Figure A.4: Illustration of Generation Fleet Ramping Capability (ramp rate vs. duration)

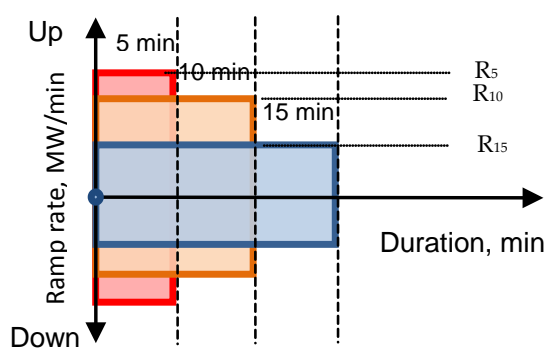




\section{Adequacy of Ramping Capability}

Generation fleet ramping capability is compared against the ramping requirements.

Figure A.5 illustrates a comparison of system ramping capability (blue curve) against ramping requirements (red curve) for some operating hours. System ramping capability is calculated using (3). Ramping requirements are calculated using the swinging door algorithm.

An area with insufficient system ramping capability is highlighted in red (Figure A.5); in other words, there is certain probability that the generation fleet does not have sufficient ramping up capability to meet the demand.

Figure A.5: Ramping Requirements vs. System Capability

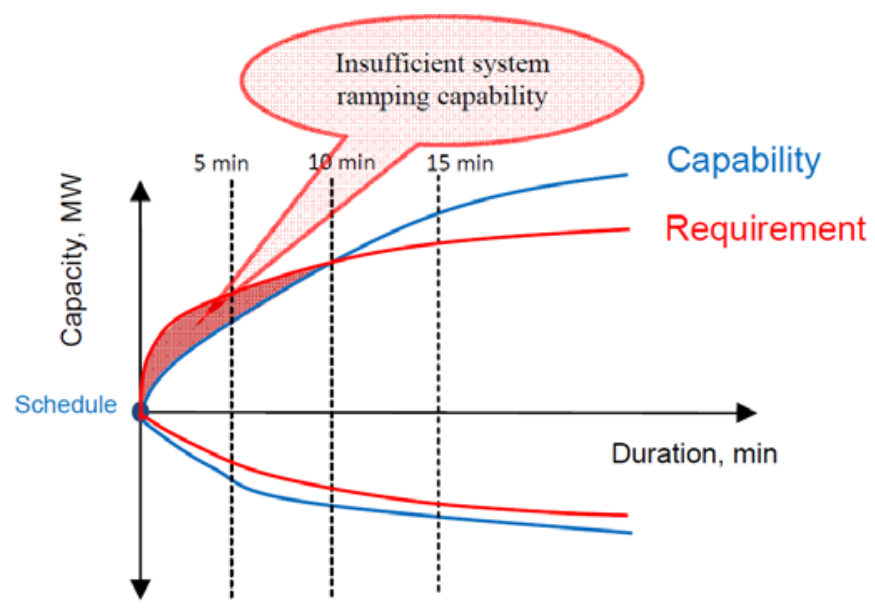




\section{APPENDIX B: Generator Forced Outage Model}

The term "generator forced outage" usually refers to the shutdown of a generating unit for emergency reasons or a condition in which the generator unit is unavailable for supplying the load because of an unanticipated breakdown. Generator outage is a discrete event and may or may not happen in any given dispatch interval. This characteristic contrasts with the continuous nature of wind and load variations. Also, the size of the power mismatch caused by a forced outage depends on the generator that is disconnected and the generators' load at the moment of the event. Any of the generators that are online within a dispatch interval could be forced out. The main challenge that has been overcome in this development was to combine the uncertainty information on continuous parameters (such as the generation capacity requirement) with discrete information (such as forced generation outages). This challenge has been successfully met in this project.

Forced outages of system generators cause temporary imbalances that must be eliminated within 10 minutes by activating the contingency reserve. Within this 10-minute interval, the system is exposed to an imbalance that can be as much as $1000 \mathrm{MW}$ (the size of the largest generation unit in the system). The system inertia, governor response, and automatic generation control act to minimize the system power mismatch during the first seconds and minutes after the disturbance. Therefore, the generation controls and generation characteristics needed to balance the system must be sufficient to mitigate these possible mismatches. Again, there is an uncertainty associated with this process because the timing and the size of the forced outages are not known ahead of time and the contingency reserve activation process is not a deterministic process (for example, it depends on the characteristics of activated generators and type of activated reserve - spinning or non-spinning).

A methodology has been developed that evaluates additional uncertainty caused by forced generator outages and incorporates this information into the overall framework. This advanced feature constitutes a significant step forward in handling the uncertainty information in the modern energy management systems (EMSs). As a result, the system reliability and control performance can be additionally improved.

Generator forced outages are stochastic events. Modeling statistical characteristics of generator forced outages is important for a correct evaluation of the future generation requirement. In the following sections, two types of generator forced outage models, i.e., the two-state Markov model and four-state Markov model, are described. The capacity outage probability table (COPT) and an example of COPT calculation are provided. Simulation results on forced outage model are also provided.

\section{Forced Outage Rate Calculation}

A generator outage is a discrete event and may or may not happen in any given hour. This feature contrasts with the continuous nature of the wind and load variations (Doherty and O’Malley 2005). 
The simplest type of the generation unit model is a two-state Markov model as shown in Figure B.1. Here the unit is assumed to always be in one of the two states: "up" - fully available, running, and subject to a possible failure; or "down" - totally unavailable, not running, and undergoing repair (Billinton and Allan 1996; Billinton and Ge 2004).

Figure B.1: Two-State Markov Model

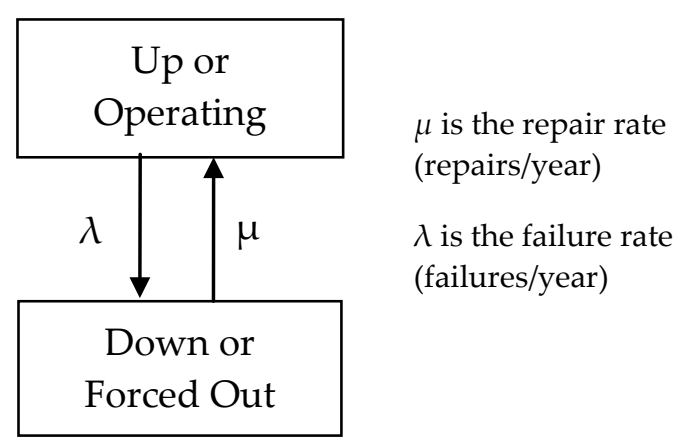

Here, $\mu$ (repairs/year) is the repair rate, $r=1 / \mu$ (years) is the mean downtime due to a forced outage (mean time to repair - MTTR), $\lambda$ is the failure rate, (failures/year), and $m=1 / \lambda$ is the mean up time between failure events (mean time to failure - MTTF). The unit's forced outage rate (FOR) is the probability that the unit is down:

$$
F O R=\frac{\lambda}{\lambda+\mu}=\frac{r}{m+r}=\frac{F O H}{S H+F O H}
$$

where $\mathrm{FOH}$ is the forced outage duration within a year, (hours), and $\mathrm{SH}$ is the service hours within a year.

The two-state model is a valid representation for base load units but does not adequately represent intermittent operating units used to meet peak load conditions. The two-state model for a base load unit has been extended to the four-state peaking unit model shown in Figure B.2, which is widely used in practice (Billinton and Ge 2004; Patton 1993). The model assumes that the generating unit is either fully available or totally unavailable, but also considers that the unit may be either needed or not needed (Billinton and Ge 2004). 
Figure B.2: IEEE Four-State Markov Model

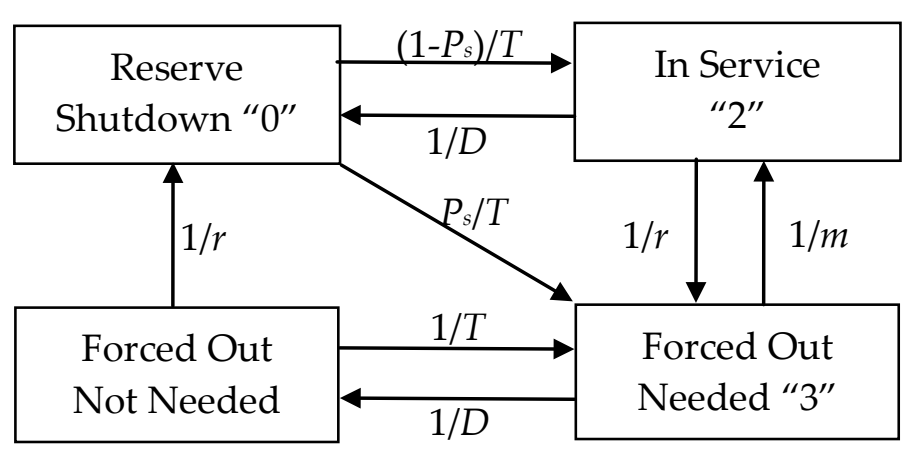

$D$ is the average in-service time per occasion of demand;

$T$ is the average reserve shutdown time between periods of need;

$r$ is the average repair time per forced outage occurrence;

$m$ is the average in-service time between occasions of forced outage when needed;

$P_{s}$ is the probability of a starting failure resulting in inability to serve load during all or part of a demand period.

The frequency balance equations for the four-state model shown in Figure B.2 are as follows (Billinton and Allan 1996; Billinton and Ge 2004):

$$
\left\{\begin{array}{l}
P_{2} \times\left(\frac{1}{D}+\frac{1}{m}\right)=P_{0} \times \frac{1-P_{s}}{T}+P_{3} \times \frac{1}{r} \\
P_{3} \times\left(\frac{1}{r}+\frac{1}{D}\right)=P_{2} \times \frac{1}{m}+P_{1} \times \frac{1}{T}+P_{0} \times \frac{P_{S}}{T} \\
P_{1} \times\left(\frac{1}{T}+\frac{1}{r}\right)=P_{3} \times \frac{1}{D} \\
P_{0}+P_{1}+P_{2}+P_{3}=1
\end{array}\right.
$$

where $P_{i}$ is the probability of the state $i, i=0 \ldots 3$.

According to Billinton and Allan (1996), $P_{1}$ and $P_{3}$ can be calculated using the following equations:

$$
\begin{gathered}
P_{1}=\frac{r^{2} T\left(D+m P_{s}\right)}{M} \\
P_{3}=\frac{r D(D+r)\left(D+m P_{s}\right)}{M}
\end{gathered}
$$

Demand factor $f$ can be expressed as the function of the parameters given in Figure B.2 as follows (Billinton and Ge 2004):

$$
f=\frac{P_{3}}{P_{3}+P_{1}}=\frac{\frac{1}{r}+\frac{1}{T}}{\frac{1}{D}+\frac{1}{r}+\frac{1}{T}}
$$

The factor $f$ serves to weight the $\mathrm{FOH}$ to reflect the time the unit was actually on forced outage when in demand by the system (Billinton and Allan 1996). 
Forced outage rate demand (FORd) can be evaluated as follows:

$$
F O R d=\frac{f \times F O H}{f \times F O H+S H}
$$

FORd is the probability that a generating unit will not be available when required.

Equivalent forced outage rate demand (EFORd) (Billinton and Allan 1996):

$$
\text { EFORd }=\frac{f \times F O H+f_{p} \times E F D H}{f \times F O H+S H}
$$

where $f_{p}$ is the partial outage factor and $\mathrm{EFDH}$ is the equivalent forced derating hours.

EFORd can be found in the NERC Generating Availability Data System (GADS) (NERC 2007; Curley 2006). The difference between EFORd and FORd is that EFORd also includes derated states of the generator.

The full outage probability (FOP) of a unit is the probability that the unit will stop providing all of its current output in an hour period. Here, it is assumed that the trip causes the unit's output to be instantaneously unavailable. The hourly FOP of a unit can be related to the FOR and MTTR as follows (Doherty and O'Malley 2005):

$$
F O P_{i}=\frac{F O R_{i}}{M T T R_{i}}
$$

In the case of peaking units, EFORd can be used instead of FOR in (B.8).

\section{Capacity Outage Probability Table}

The capacity adequacy evaluation of generation systems requires the creation of a generation capacity model, known as the capacity outage probability table (COPT). COPT gives the probability of occurrence for each possible outage capacity level (Billinton and Allan 1996).

Let us assume that the system has $n$ independent generating units and that unit $i$ has $m_{i}$ discrete states with outage capacity $C_{i j}$ and individual probability $p_{i j}=p\left(X_{i}=C_{i j}\right)$, where $j=1 \ldots m_{i}$ (Morrow and Gan 1993). Outage states of unit $i$ are arranged in ascending order. The COPT contains $N+1$ discrete states, where $N=C_{\max } / \Delta, C_{\max }$ is the installed capacity of the system and $\Delta$ is the resolution of the COPT. The new individual state probabilities, after unit $i$ is added to the system, can be calculated using the following recursive algorithm (Morrow and Gan 1993):

$$
p(k)=\sum_{j=1}^{m_{i}} p_{i j} p^{\prime}\left(k-\frac{c_{i j}}{\Delta}\right), \quad k=0,1,2, \ldots, N
$$

where $p(\cdot)$ is individual state probabilities after unit $i$ is added; $p^{\prime}(\cdot)$ is individual state probabilities before unit $i$ is added; and $k$ is an index of discrete state. 
The recursive convolution process starts with the initial values: $p(0)=1$ and $p(k)=0, k=1,2, \ldots N$. Note that $p(k)=0$ if $k<0$.

In summary, the recursive convolution procedure for building a COPT has the following basic steps (Morrow and Gan 1993):

- Read unit data, determine $\Delta$ and $N=C_{\max } / \Delta$;

- Set initial values: $p(0)=1$ and $p(k)=0, k=1,2, \ldots N$;

- Add unit $i$ to the system, calculate $p(k), k=0,1,2, \ldots, N$ using (B.8);

- $\quad$ Repeat Step 3 for all the units.

Usually, the table obtained by (B.8) is simplified by rounding the COPT to selected discrete capacity levels. The size of the round-off increment depends on the desired accuracy.

The cumulative probability of having $k \Delta \mathrm{MW}$ to be forced out can be calculated using the following equation:

$$
P(k)=\sum_{s=0}^{k} p(s)
$$

\section{Example of COPT Calculation}

Let the system consist of two generators. The first generator has a capacity of $100 \mathrm{MW}$ and outage probability $10 \%$, and the second generator has a capacity of $50 \mathrm{MW}$ and outage probability $20 \%$. Assume that generating units has only two states: operating state and forced out state.

Then, the capacity matrix is:

$$
C=\left[\begin{array}{ll}
C_{11} & C_{12} \\
C_{21} & C_{22}
\end{array}\right]=\left[\begin{array}{cc}
0 & 100 \\
0 & 50
\end{array}\right]
$$

where $c_{11}=0$ and $c_{21}=0$ - correspond to operating states of generators one and two (no forced outage) and $c_{12}=100$ and $c_{22}=50$ - correspond to forced out states (nominal generator capacity).

Individual probability matrix is defined as:

$$
P=\left[\begin{array}{ll}
p_{11} & p_{12} \\
p_{21} & p_{22}
\end{array}\right]=\left[\begin{array}{ll}
0.9 & 0.1 \\
0.8 & 0.2
\end{array}\right],
$$

where $p_{11}=0.9$ and $p_{21}=0.8$ are probabilities of operating state of generators one and two; and $p_{12}=0.1$ and $p_{22}=0.2$ are probabilities of the forced out state.

The installed system capacity is $C_{\max }=150 \mathrm{MW}$, and the COPT resolution is $\Delta=50 \mathrm{MW}$. Therefore, COPT contains four discrete states.

Let us set initial probability values $p(k)$ in the COPT (Table B.1). 
Table B.1. COPT (Initial Values)

\begin{tabular}{lll}
\hline State, $k$ & Capacity, $c(k)(\mathrm{MW})$ & Probability, $p(k)$ \\
\hline 0 & 0 & 1 \\
1 & 50 & 0 \\
2 & 100 & 0 \\
3 & 150 & 0
\end{tabular}

Now we will add unit one to the system and calculate new capacity outage probabilities using (B.8) - see Table B.2

$$
\begin{aligned}
k=0: \quad p(0) & =p_{11} \cdot p^{\prime}\left(k-\frac{c_{11}}{\Delta}\right)+p_{12} \cdot p^{\prime}\left(k-\frac{c_{12}}{\Delta}\right)=0.9 \cdot p^{\prime}\left(0-\frac{0}{50}\right)+0.1 \cdot p^{\prime}\left(0-\frac{100}{50}\right)= \\
= & 0.9 \cdot p^{\prime}(0)+0.1 \cdot p^{\prime}(-2)=0.9 \cdot 1+0.1 \cdot 0=0.9 \\
k=1: \quad p(1) & =p_{11} \cdot p^{\prime}\left(k-\frac{c_{11}}{\Delta}\right)+p_{12} \cdot p^{\prime}\left(k-\frac{c_{12}}{\Delta}\right)=0.9 \cdot p^{\prime}\left(1-\frac{0}{50}\right)+0.1 \cdot p^{\prime}\left(1-\frac{100}{50}\right)= \\
& =0.9 \cdot p^{\prime}(1)+0.1 \cdot p^{\prime}(-1)=0.9 \cdot 0+0.1 \cdot 0=0 \\
k=2: \quad p(2) & =p_{11} \cdot p^{\prime}\left(k-\frac{c_{11}}{\Delta}\right)+p_{12} \cdot p^{\prime}\left(k-\frac{c_{12}}{\Delta}\right)=0.9 \cdot p^{\prime}\left(2-\frac{0}{50}\right)+0.1 \cdot p^{\prime}\left(2-\frac{100}{50}\right)= \\
& =0.9 \cdot p^{\prime}(2)+0.1 \cdot p^{\prime}(0)=0.9 \cdot 0+0.1 \cdot 1=0.1 \\
k=3: \quad p(3) & =p_{11} \cdot p^{\prime}\left(k-\frac{c_{11}}{\Delta}\right)+p_{12} \cdot p^{\prime}\left(k-\frac{c_{12}}{\Delta}\right)=0.9 \cdot p^{\prime}\left(3-\frac{0}{50}\right)+0.1 \cdot p^{\prime}\left(3-\frac{100}{50}\right)= \\
& =0.9 \cdot p^{\prime}(3)+0.1 \cdot p^{\prime}(1)=0.9 \cdot 0+0.1 \cdot 0=0
\end{aligned}
$$

Table B.2: COPT (Unit One Added)

\begin{tabular}{lll}
\hline State, $k$ & Capacity, $c(k)(\mathrm{MW})$ & Probability, $p(k)$ \\
\hline 0 & 0 & 0.9 \\
1 & 50 & 0 \\
2 & 100 & 0.1 \\
3 & 150 & 0 \\
\hline
\end{tabular}


The next step is adding the unit two and update values of COPT (Table B.3):

$$
\begin{aligned}
k=0: \quad p(0) & =p_{21} \cdot p^{\prime}\left(k-\frac{c_{21}}{\Delta}\right)+p_{22} \cdot p^{\prime}\left(k-\frac{c_{22}}{\Delta}\right)=0.8 \cdot p^{\prime}\left(0-\frac{0}{50}\right)+0.2 \cdot p^{\prime}\left(0-\frac{50}{50}\right)= \\
= & 0.8 \cdot p^{\prime}(0)+0.2 \cdot p^{\prime}(-1)=0.8 \cdot 0.9+0.2 \cdot 0=0.72 \\
k=1: \quad p(1) & =p_{21} \cdot p^{\prime}\left(k-\frac{c_{21}}{\Delta}\right)+p_{22} \cdot p^{\prime}\left(k-\frac{c_{22}}{\Delta}\right)=0.8 \cdot p^{\prime}\left(1-\frac{0}{50}\right)+0.2 \cdot p^{\prime}\left(1-\frac{50}{50}\right)= \\
& =0.8 \cdot p^{\prime}(1)+0.2 \cdot p^{\prime}(0)=0.8 \cdot 0+0.2 \cdot 0.9=0.18 \\
k=2: \quad p(2) & =p_{21} \cdot p^{\prime}\left(k-\frac{c_{21}}{\Delta}\right)+p_{22} \cdot p^{\prime}\left(k-\frac{c_{22}}{\Delta}\right)=0.8 \cdot p^{\prime}\left(2-\frac{0}{50}\right)+0.2 \cdot p^{\prime}\left(2-\frac{50}{50}\right)= \\
& =0.8 \cdot p^{\prime}(2)+0.2 \cdot p^{\prime}(1)=0.8 \cdot 0.1+0.2 \cdot 0=0.08 \\
k=3: \quad p(3) & =p_{21} \cdot p^{\prime}\left(k-\frac{c_{21}}{\Delta}\right)+p_{22} \cdot p^{\prime}\left(k-\frac{c_{22}}{\Delta}\right)=0.8 \cdot p^{\prime}\left(3-\frac{0}{50}\right)+0.2 \cdot p^{\prime}\left(3-\frac{50}{50}\right)= \\
& =0.8 \cdot p^{\prime}(3)+0.2 \cdot p^{\prime}(2)=0.8 \cdot 0+0.2 \cdot 0.1=0.02
\end{aligned}
$$

Table B.3: COPT (Unit Two Added)

\begin{tabular}{lll}
\hline State, $k$ & Capacity, $c(k)(\mathrm{MW})$ & Probability, $p(k)$ \\
\hline 0 & 0 & 0.72 \\
1 & 50 & 0.18 \\
2 & 100 & 0.08 \\
3 & 150 & 0.02 \\
\hline
\end{tabular}

Figures B.3-B.4 show the capacity discrete outage probability density function (PDF) and cumulative distribution function (CDF) based on the calculated COPT. 
Figure B.3: Discrete Probability Density Function

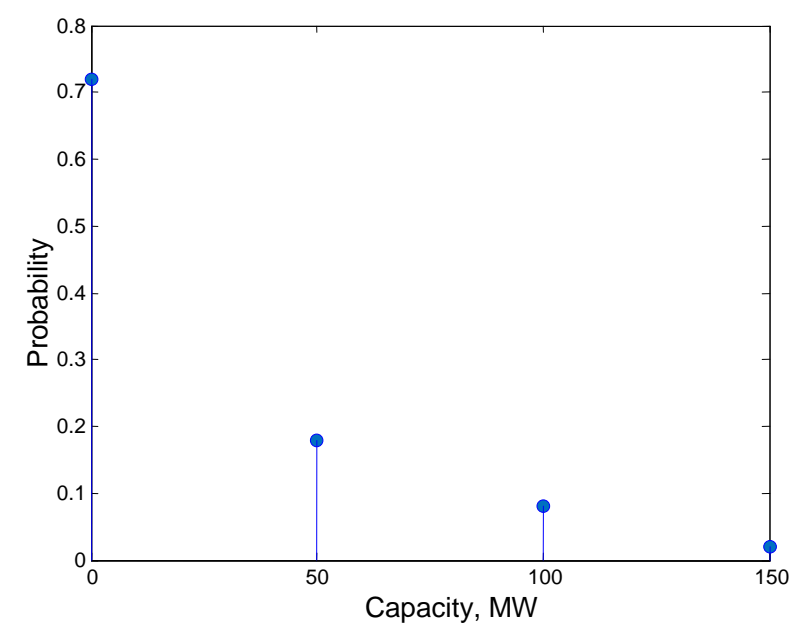

Figure B.4: Cumulative Distribution Function

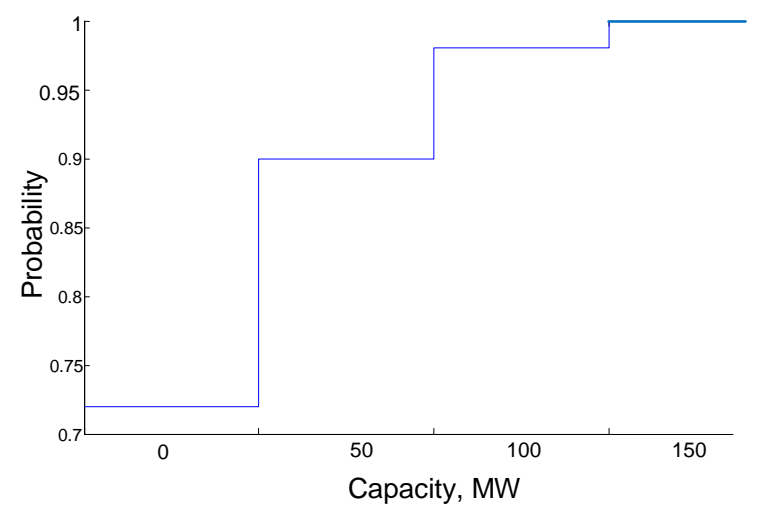

\section{Simulation Results (Forced Outage Model)}

An example of a California Independent System Operator (CAISO) generation schedule is presented in Table B.4, and generation unit performance statistical characteristics taken from GADS (NERC 2007) are presented in Table B.5.

COPTs are calculated to each hour, taking into account the generators' schedule. Figures B.5-B.6 show the capacity outage PDF and CDF functions for a 1-hour look-ahead period. 
Table B.4: Generation Schedule

\begin{tabular}{llllllll}
\hline Number & UNIT_ID & Unit Type & $1 \mathrm{~h}$ & $2 \mathrm{~h}$ & $3 \mathrm{~h}$ & $4 \mathrm{~h}$ & $5 \mathrm{~h}$ \\
\hline 1 & Unit1 & STUR & 16 & 16 & 16 & 16 & 16 \\
2 & Unit2 & STUR & 20 & 20 & 20 & 20 & 20 \\
3 & Unit3 & HYDR & 16 & 16 & 16 & 16 & 16 \\
4 & Unit4 & GTUR & 0 & 0 & 0 & 0 & 0 \\
$\ldots \ldots$ & $\ldots$ & $\ldots \ldots$ & $\ldots$ & $\ldots$ & $\ldots$ & $\ldots$ & $\ldots$ \\
516 & Unit516 & STUR & 3 & 3 & 3 & 3 & 3 \\
517 & Unit517 & WIND & 10 & 10 & 10 & 10 & 10 \\
& Total Generation & & 17792.9 & 16512.06 & 16113.22 & 15813.15 & 15811.15 \\
& Wind & 1344 & 1310.28 & 1313.55 & 1299.14 & 1256.3 \\
\hline GTUR & $=$ Gas turbine & & & & & \\
HYDR $=$ Hydro & & & & & & \\
STUR $=$ Stem turbine & & & & & & & \\
WIND $=$ Wind & & & & & & &
\end{tabular}

Table B.5: Annual Unit Performance Statistic

\begin{tabular}{llllll}
\hline GEN_TYPE & GEN_TECH & FUEL_TYPE & FOR & Service Hours & Number of Occurrences \\
\hline $\mathrm{T}$ & STUR & GEOT & 0.5 & 8500 & 3.6 \\
$\mathrm{~T}$ & GTUR & GAS & 46.33 & 270 & 3 \\
$\mathrm{~T}$ & STUR & GAS & 8.29 & 2750 & 4 \\
$\mathrm{H}$ & HYDR & WATR & 4.92 & 4981 & 3 \\
$\mathrm{~T}$ & WIND & WIND & - & - & - \\
$\mathrm{T}$ & CCYC & GAS & 7.33 & 3673 & 9 \\
$\mathrm{H}$ & PTUR & WATR & 3.71 & 2634 & 3.86 \\
\hline
\end{tabular}

CCYC = Combined cycle

GAS $=$ Gas

GEOT $=$ Geothermal

GTUR $=$ Gas turbine

HYDR = Hydro

STUR = Steam turbine

WATR $=$ Water

WIND $=$ wind 
Figure B.5: Capacity Outage Discrete PDF

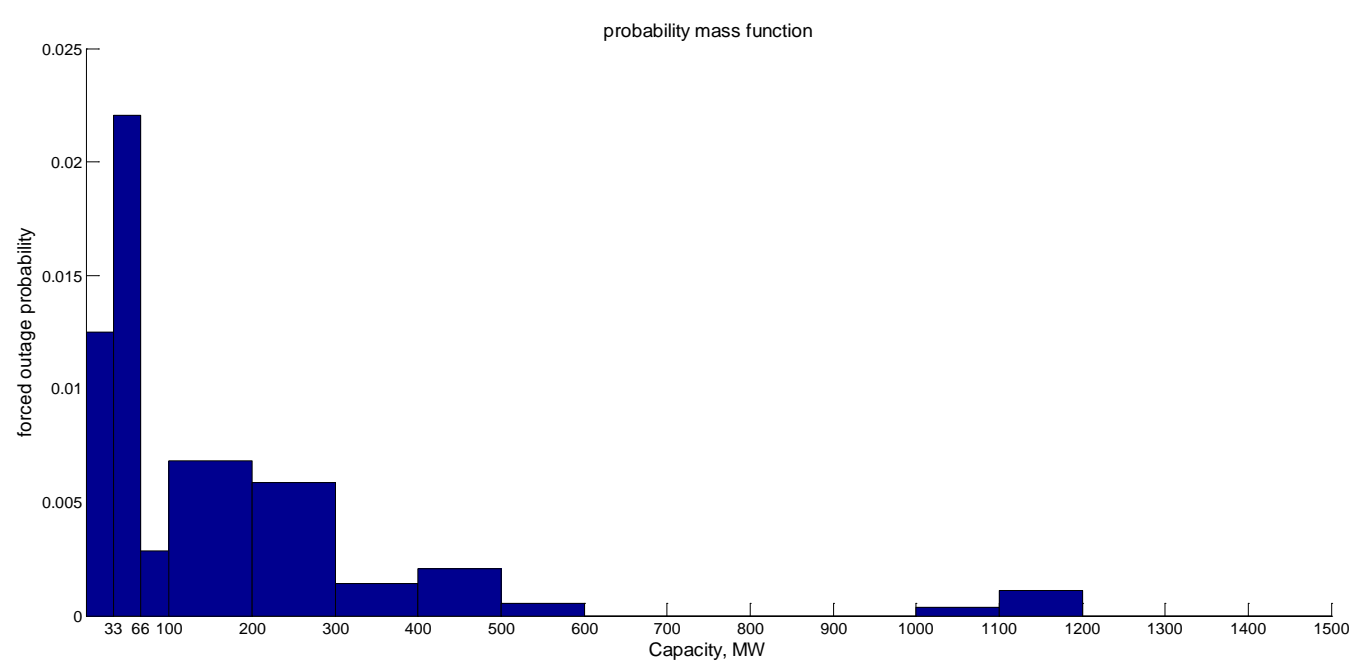

Figure B.6: Capacity Outage CDF

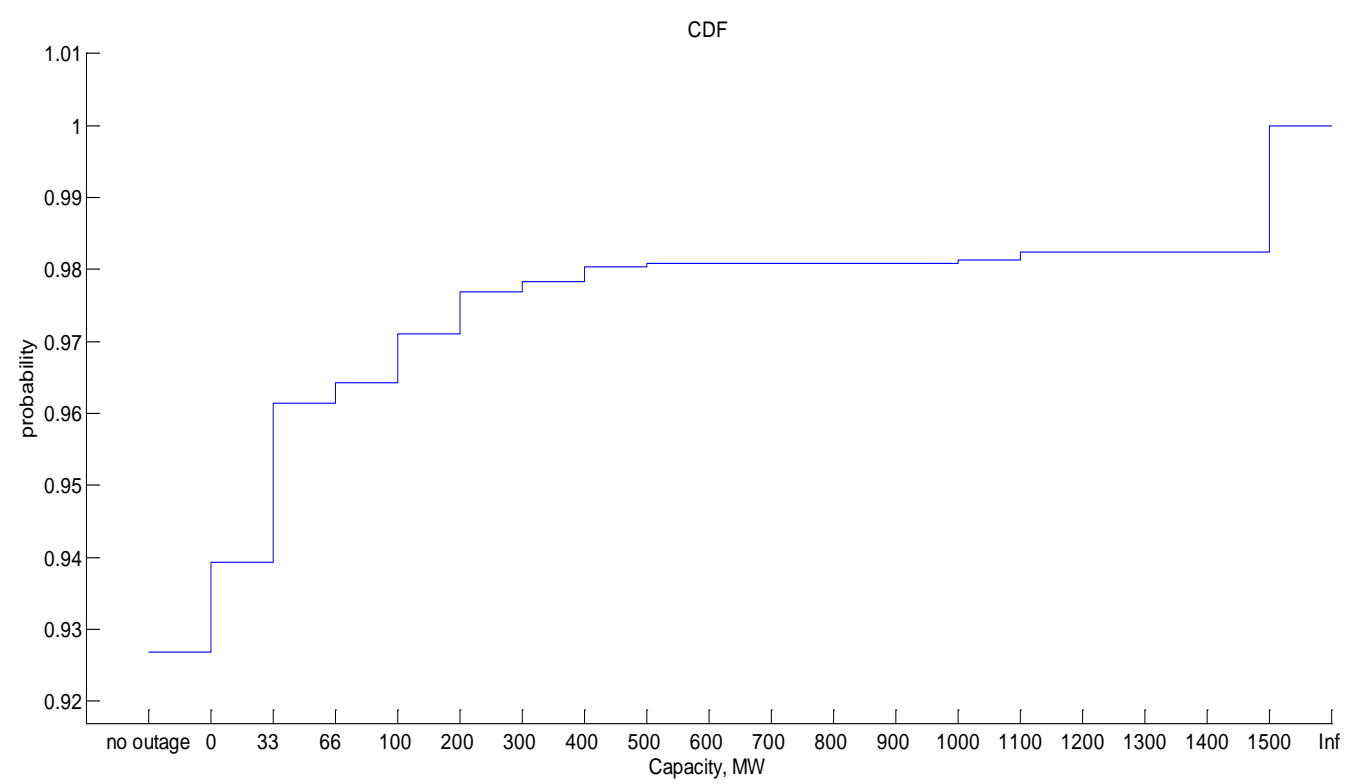

\section{References}

Billinton R., and J. Ge. 2004. “A Comparison of Four-state Generating Unit Reliability Models for Peaking Units." IEEE Trans. on Power Syst. 19:763-768.

Billinton, R., and R. Allan. 1996. Reliability Evaluation of Power System, Plenum Press, New York.

Curley, G. M. 2006. “Power Plant Performance Indices in New Market Environment." IEEE Standard 762 Working Group Activities and GADS Database. Proc. IEEE Power Engineering Society General Meeting, p. 5. 
Doherty, R., and M. O'Malley. 2005. "A new approach to quantify reserve demand in systems with significant installed wind capacity." IEEE Trans. on Power Syst. 20:587-595.

Morrow, D., and L. Gan. 1993. "Comparison of Methods for Building a Capacity Model in Generation Capacity Adequacy Studies." Proc. IEEE WESCANEX'93, Communications, Computers and Power in the Modern Environment, pp. 143-149.

NERC - North American Electric Reliability Corporation. 2007. “Generating Availability Data System Report." North American Electric Reliability Corporation. [Online.] Available: http://www.nerc.com/files/gar2007.zip.

Patton, A. D. 1993. “Duty Cycle Effects on Generating Unit Availability.” IEEE Trans. on Industry Applications 29:1018-1021. 


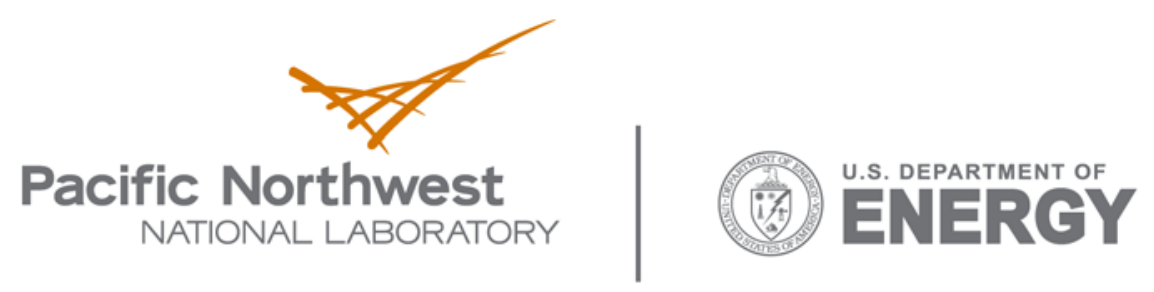

902 Battelle Boulevard

P.O. Box 999

Richland, WA 99352

1-888-375-PNNL (7665)

www.pnl.gov 